\title{
Investigating the role of the growth hormone secretagogue receptor in food-motivated behaviours
}

\author{
by \\ Samantha J. King \\ A thesis submitted to the Faculty of Graduate and Postdoctoral Affairs in partial \\ fulfillment of the requirements for the degree of \\ Doctor of Philosophy \\ in \\ Neuroscience \\ Carleton University \\ Ottawa, ON \\ (C) 2018 Samantha King
}




\begin{abstract}
.
Ghrelin is a peptide produced in the gut that stimulates food intake. Action of ghrelin on its receptor, the GHSR, contributes to food motivated behaviours. Studies have found that the GHSR facilitates the activity of mesolimbic DA circuitry, promoting intake of highly palatable sugar or fat rich foods. Understanding what makes such foods attractive and rewarding may allow for behavioural, genetic, or pharmacological therapeutics to mitigate problematic overconsumption. The primary goals of this dissertation were to determine the consequences of interrupted GHSR signaling on food motivated behaviours, including binge-like feeding, and to examine whether GHSR in the VTA are an important neural substrate for the development and maintenance of such behavior. Operant responding for sucrose on a PR schedule of reinforcement, a measure of motivation to obtain a food reward, was diminished when rats lacked functional GHSR. These rats also spent less time in an environment previously paired with sucrose, suggesting a possible reward deficit as a result of loss of GHSR. GHSR null mice also consumed less sucrose in a two-bottle choice test in their home cage, and rescue of GHSR in the VTA was able to restore their preference for sucrose to the level of WT mice, implicating the VTA as a key node for GHSRs influence on preference for sweets. In a limited access model of high-fat binge eating, we found that GHSR KO mice binged less on fat than WT mice. Though, in our subsequent study, we found less robust effects of genotype on binge eating behavior, and rescue of GHSR in the VTA did not influence binge eating of fat. Further, pharmacological antagonism of the GHSR was not sufficient to reduce binge eating of high-fat in mice once established, indicating that the VTA is not the primary site of GHSRs influence on fat bingeing. Further studies examining ghrelin and the GSHR as moderators of food reward behavior are warranted, as it is likely that signaling through the GHSR promotes the associative learning induced by palatable food cues, as well as the motivation to obtain such foods, which may facilitate overconsumption.
\end{abstract}




\section{Acknowledgements.}

I would like to thank my supervisor, Dr. Alfonso Abizaid, for the guidance, encouragement and advice he has provided me during my time in his lab. I consider myself lucky to have worked under his supervision and appreciate the freedom he offered me to pursue my research interests. His patience and compassion also helped me continue at times I thought I could not succeed. I would also like to thank Dr. Barbara Woodside for her assistance with my thesis, and for the much needed data/coffee dates. Her help was invaluable, and I greatly appreciate it. I'd also like to thank all of the other faculty and staff members in the Department of Neuroscience who have been there for me every step of the way, especially Diane Trenouth and Heather Martel. Thank you to Dr. John Stead and Dr. Kim Hellemans for giving me the opportunity to teach and foster a love of neuroscience in our undergrads, and to Dr. Matt Holahan for his advice on how to navigate student issues.

I could not have done this without the constant support from my lab mates. Thank you to Dr. Zach Patterson, the first student I met in the lab, for providing early morning chats (much to my chagrin), scientific expertise and humor. To Dr. Rim Khazall, for being my friend, a great collaborator, a constant source of emotional support, and most importantly for bringing me food and coffee. I truly could not have done this without you. To Su-Bin Park, the 'giggly one', my surgical partner-in-crime, and a great friend, thank you for everything. Thank you to Dr. Martin Wellman, for providing an endless wealth of knowledge and for bringing me weekly produce approximating the size of the baby in utero during my first pregnancy, that's commitment! Thank you to Cathy Nesbitt, Alex Edwards, and Lindsay Hyland, for lending a hand during my experiments, being great friends, and helping me get to the finish line. Thank you to Eliza for being persistently optimistic and always having a smile on your face, no matter what. I'd also like to thank all of the undergrads who have worked with me over the years, especially Albert, Emma, Alex, Anne, Logan, Troy, and Gabby, who themselves have gone on to do amazing things.

Thank you to my family (especially my mom) who provide endless support, love and encouragement. Thank you to Marianne and Kim and the rest of the clan for helping with the kids and with daily logistics that allowed me to get my work done. I will be forever grateful. Finally, thank you to my wonderful and loving husband, who does everything for our family so that I can focus and remain sane. You are the reason I made it. I am forever in your debt. And to countless others (you know who you are!) thank you for believing in me. 


\section{Dedication.}

This thesis is dedicated to my dad, who always said to never underestimate the value of an education. And to my boys, who are always waiting with the biggest smiles when I come home and make the world much brighter. I love you. 


\section{List of Abbreviations.}

$\alpha$-MSH: $\alpha$-melanocyte stimulating hormone

ACTH: adrenocorticotropin hormone

AgRP: agouti related peptide

AAV: adeno-associated virus

AAV-Cre: adeno-associated virus containing cre-recombinase

AAV-eGFP: adeno-associated virus containing green fluorescent protein

AN: anorexia

ANOVA: analysis of variance

ARC: arcuate nucleus of the hypothalamus

BED: binge eating disorder

BIM28163: ghrelin analog; ghrelin receptor antagonist

$\mathrm{BN}$ : bulimia nervosa

BP: break point

BSA: bovine serum albumen

CART: cocaine and amphetamine related transcript

CCAC: Canadian Council on Animal Care

CNS: central nervous system

CORT: corticosterone

CPP: conditioned place preference

CRE: cre-recombinase

CS: conditioned stimulus

CVO: circumventricular organs

DA: dopamine

DAILY: daily access to high-fat

DAB: diaminobenzidine

DAG: des-acyl ghrelin

DAPI: 4' 6' diamidino-2-phenylindole

DIO: diet-induced obesity

EW: Edinger Westphal nucleus

ENU: N-ethyl-N-nitrosourea

FHH: Fawn-Hooded Hypertensive

FR: fixed ratio

GABA: $\gamma$-aminobutyric acid

GC: glucocorticoid

GFP-IF: GFP immunofluorescence

$\mathrm{GH}$ : growth hormone

GHSR: growth hormone secretagogue receptor (ghrelin receptor)

GHSR KO: GHSR knock out

GOAT: ghrelin-o-acyltransferase 
GPCR: G-protein-coupled receptor

HFD: high fat diet

ICV: intracerebroventricular

IHC: immunohistochemistry

INT: intermittent

IP: intraperitoneal

IR: immunoreactivity

JMV-2959: ghrelin receptor antagonist

Kcal: kilocalories

LH: lateral hypothalamus

MC3R: melanocortin-3 receptor

MC4R: melanocortin-4 receptor

ME: median eminence

MePV: posteroventral medial amygdala

MOR: mu opioid receptor

NAcc: nucleus accumbens

NAcSh: nucleus accumbens shell

NGS: normal goal serum

NPY: neuropeptide Y

NTS: nucleus tractus solitarus

PB: phosphate buffer

PCR: polymerase chain reaction

PFA: paraformaldehyde

PFC: prefrontal cortex

POMC: proopiomelanocortin

PR: progressive ratio

PVN: paraventricular nucleus

SEM: standard error of the mean

TBC: transcriptional blocking cassette

TD: test day

TH: tyrosine hydroxylase

TH-IF: TH immunofluorescence

UCS: unconditioned stimulus

VMH: ventromedial hypothalamus

VTA: ventral tegmental area

WAT: white adipose tissue

WT: wild type 


\section{Table of Contents}

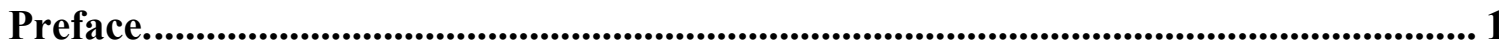

Chapter 1. The role of ghrelin in the central regulation of feeding. ........................... 4

Regulation of feeding behavior in mammals. .............................................................................

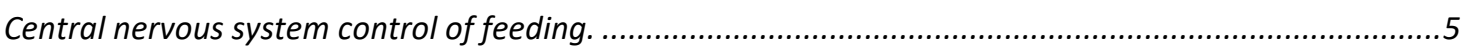

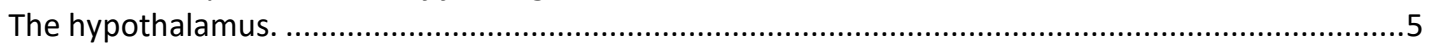

Peptide regulation of food intake in the hypothalamus........................................................... 7

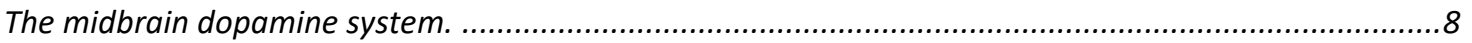

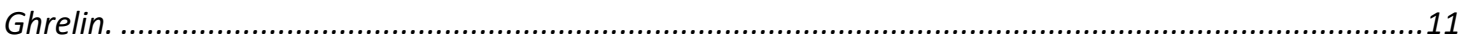

Ghrelin and dopamine in the regulation of food reward. ......................................................13

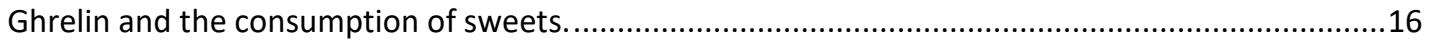

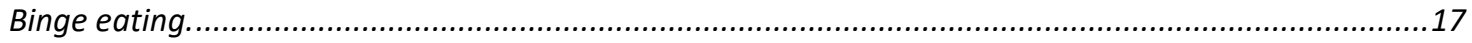

Using genetic knock-out and adeno-associated viral delivery of cre to evaluate the necessity of GHSR in

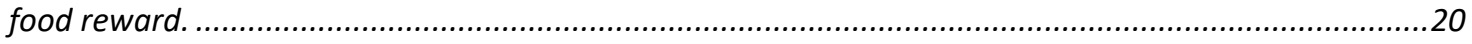

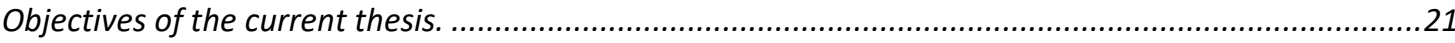

Chapter 2: Rats and mice without functional GHSR exhibit reduced motivation and attenuated preference for sucrose reward, the latter being augmented by rescue of

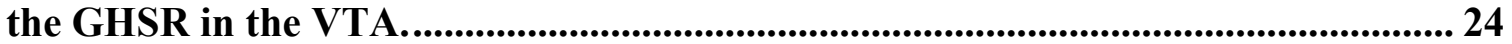

Introduction.

\section{Experiment 1.1. Hungry GHSR ${ }^{\mathrm{m} 1 / \mathrm{Mcwi}}$ rats are less motivated to obtain sucrose} pellets under a progressive ratio schedule of responding......................................... 28

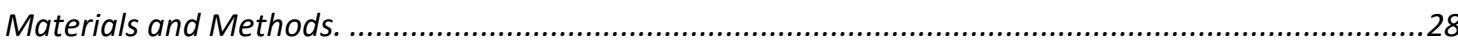

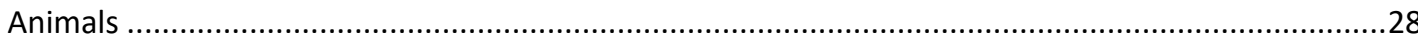

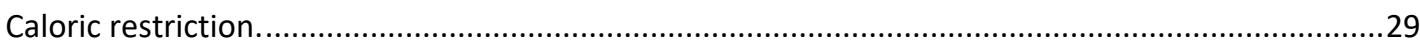

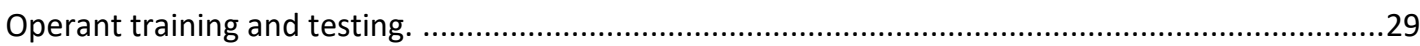

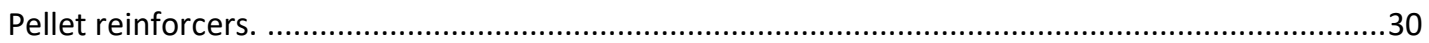

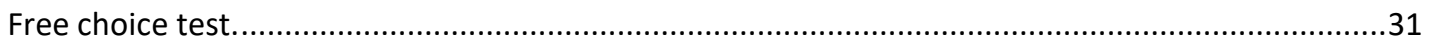

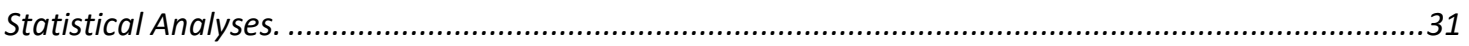

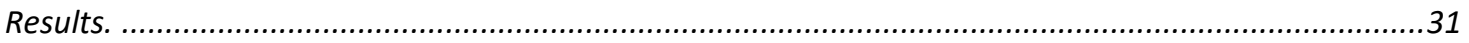

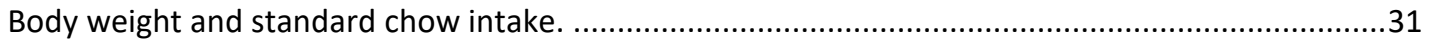

$\mathrm{GHSR}^{\mathrm{m} 1 / \mathrm{Mcwi}}$ and WT rats did not differ on Fixed ratio 4 responding (FR4) for regular, chocolate

flavoured, sucrose or high-fat chocolate flavoured pellets. ........................................................33

GHSRm1/Mcwi rats were less motivated to obtain sucrose pellets under a progressive ratio

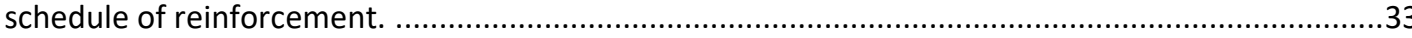

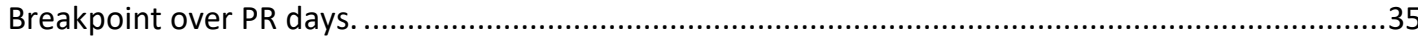

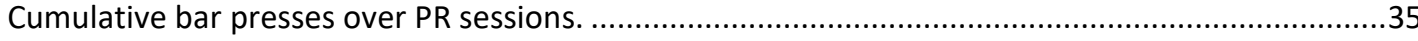

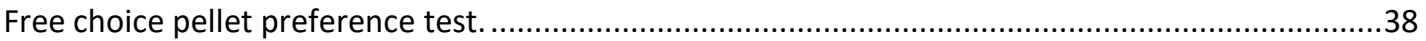

Experiment 1.2: GHSR $^{\mathrm{m} 1 / \mathrm{Mcwi}}$ rats spend less time in a sugar-paired compartment when conditioned in a hungry state, but when satiated during testing. ..................... 39

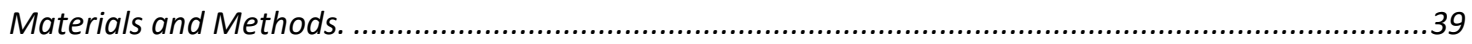




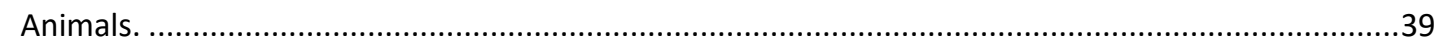

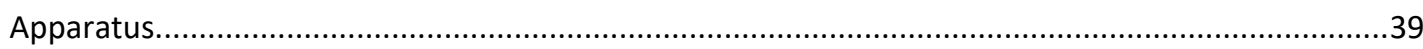

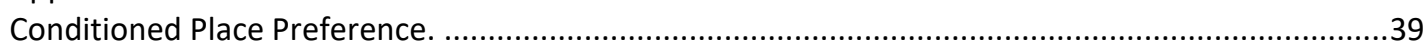

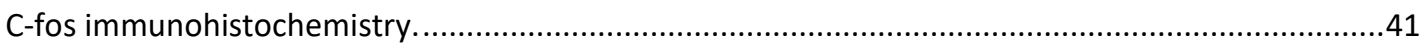

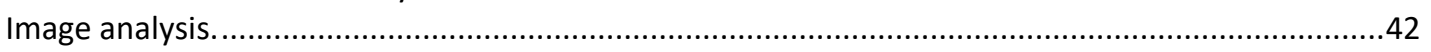

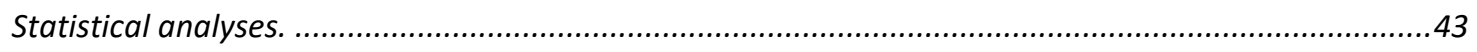

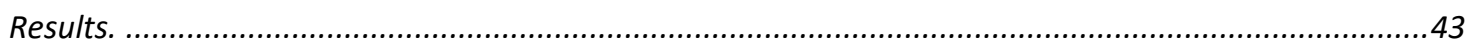

GHSRm1/Mcwi rats spend significantly less time in environments previously paired with sucrose,

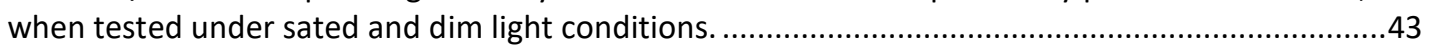

GHSR $^{\mathrm{m} 1 / \mathrm{Mcwi}}$ rats display attenuated cFos expression in the ventral tegmental area. ........................44

$\mathrm{GHSR}^{\mathrm{m} 1 / \mathrm{Mcwi}}$ animals display augmented C-fos expression in the posteroventral medial amygdala

(MePV)......

GHSRm1/Mcwi and WT rats exhibited similar levels of C-fos expression in the NAcc core and shell regions.

Experiment 1.3.1: Demonstrating localization of AAV infusion using greenfluorescent protein and tyrosine hydroxylase immunohistochemistry........................ 46

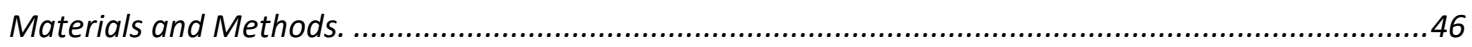

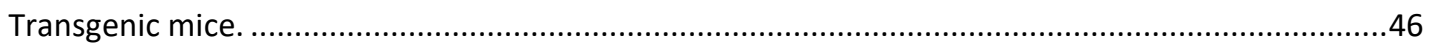

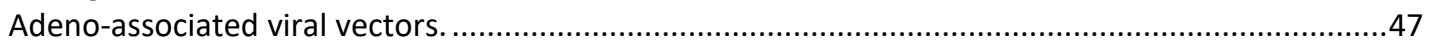

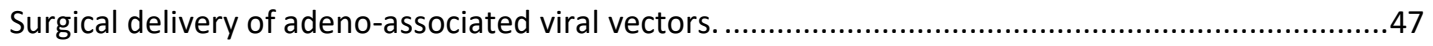

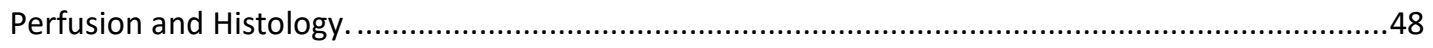

Immunohistochemical verification of viral transduction.............................................................. 48

\section{Experiment 1.3.2: Rescue of the GHSR in the VTA enhances preference for sucrose} solutions in a two-bottle choice test. ...................................................................................... 51

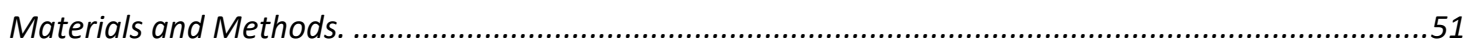

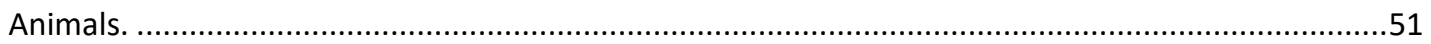

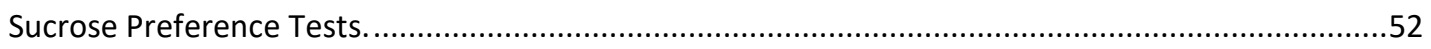

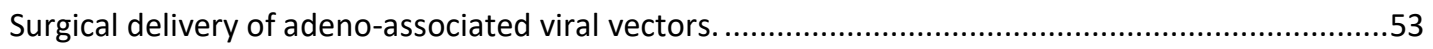

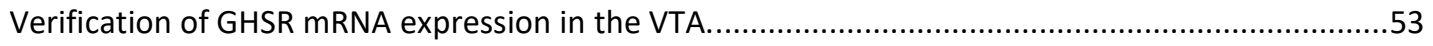

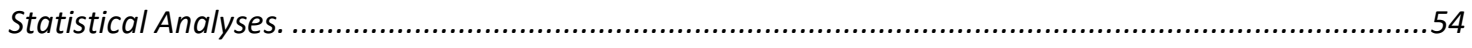

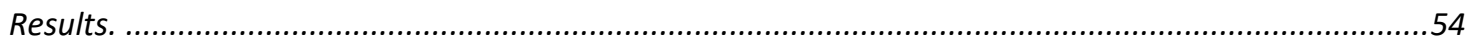

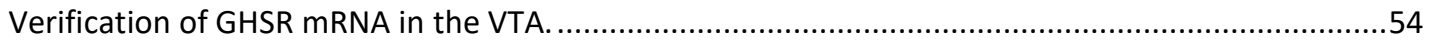

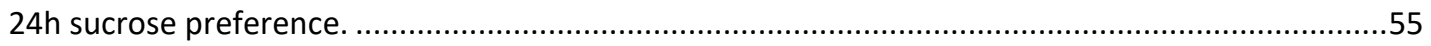

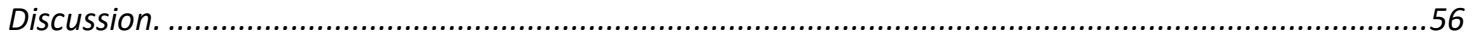

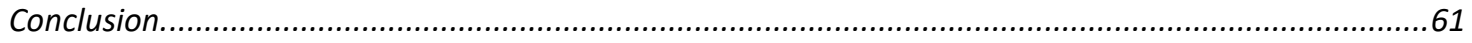

Chapter 3: Investigation of a role for ghrelin signaling in binge-like feeding in mice under limited access to high-fat diet (Studies 2.1, 2.2)............................................. 67

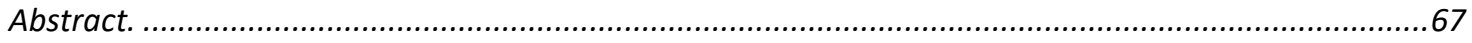

Introduction.

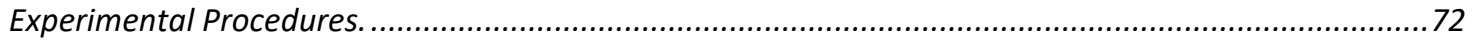

Animals.

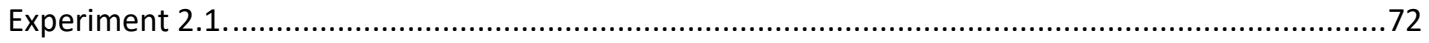

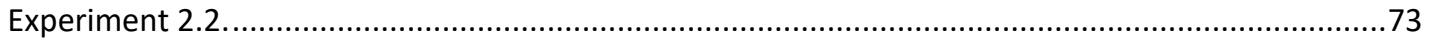




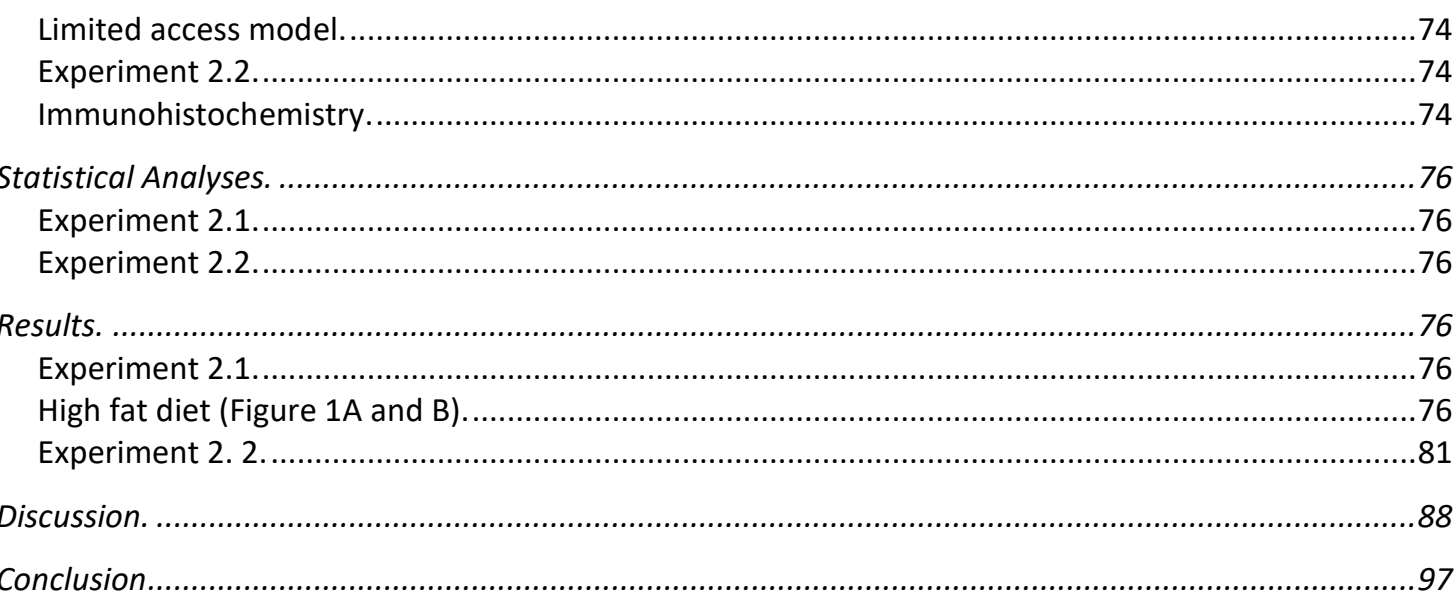

Chapter 4: Exploring the contribution of the GHSR in the ventral tegmental area (VTA) on binge-like consumption of a high-fat diet (Studies 2.3 and 2.4). .............. 99

Introduction.

\section{Experiment 2.3. Adeno-associated viral rescue of the GHSR and its influence on} high-fat bingeing under the limited access model............................................................. 105

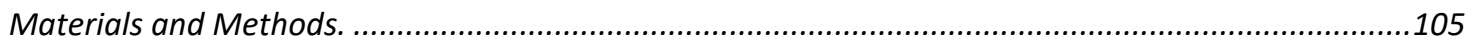

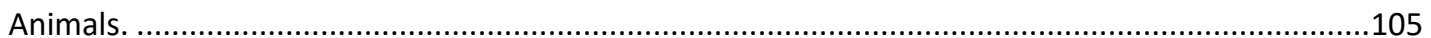

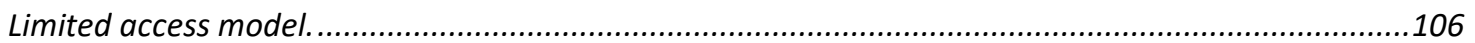

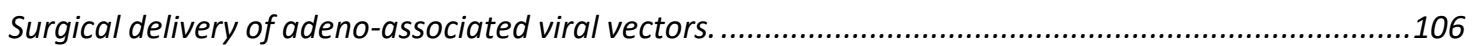

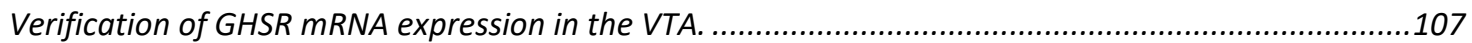

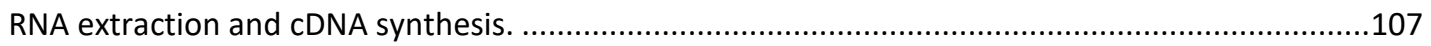

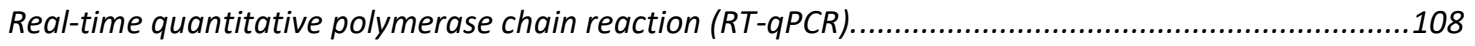

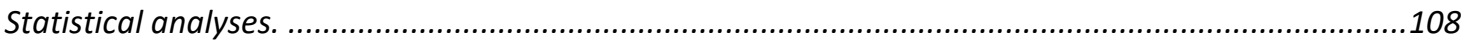

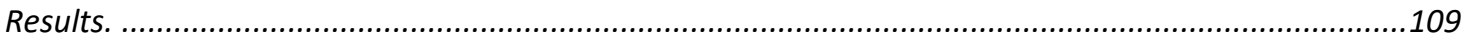

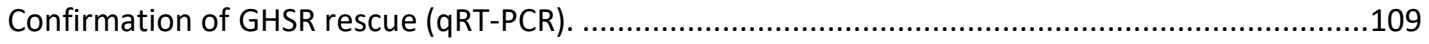

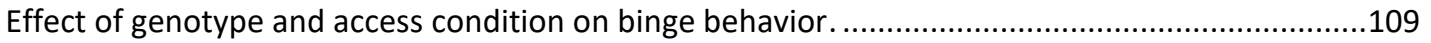

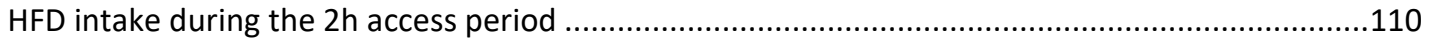

Effect of genotype and access condition on Chow intake ...........................................................111

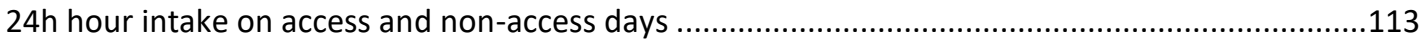

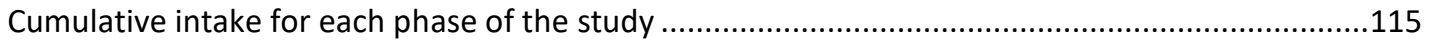

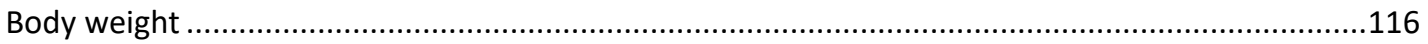

Experiment 2.4. Antagonism of the GHSR in the VTA to attenuate HFD following the establishment of binge-like intake................................................................................. 117

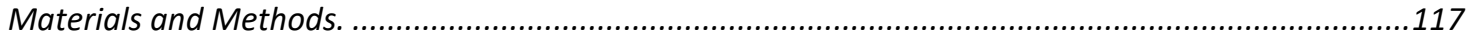

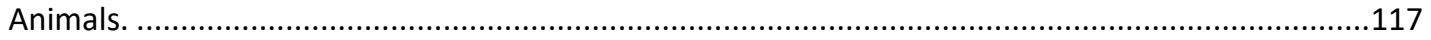

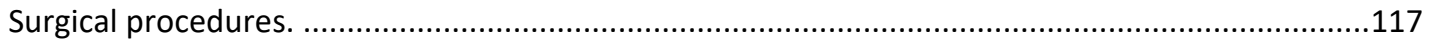

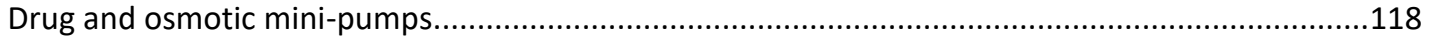

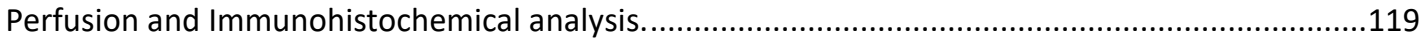

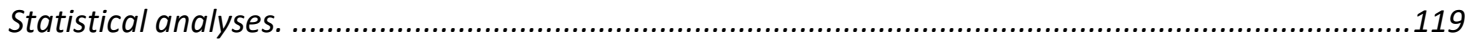




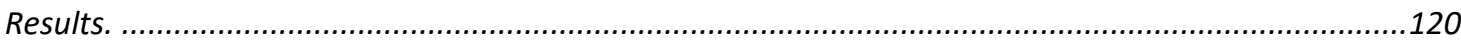

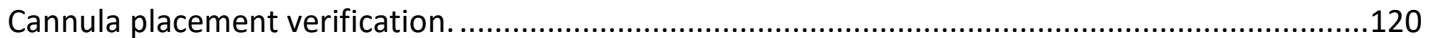

Effect of genotype and access condition on binge behavior. ......................................................120

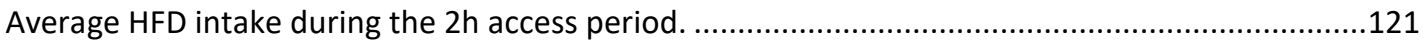

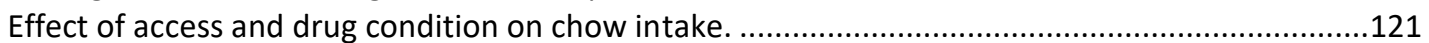

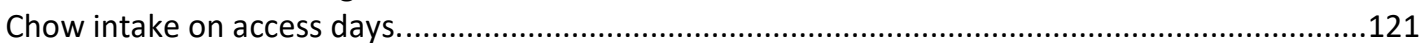

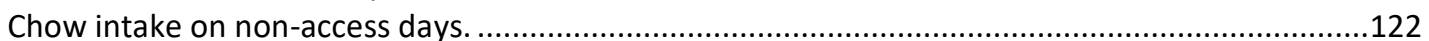

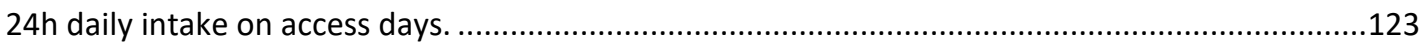

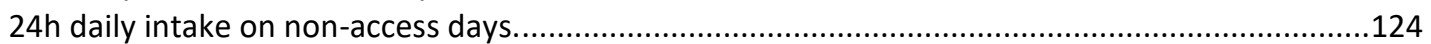

Cumulative intake and dietary composition over the pre- and post-surgery periods.......................124

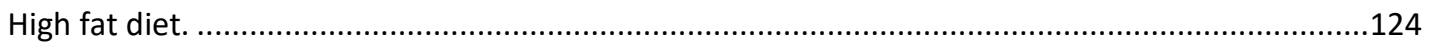

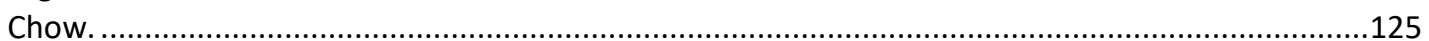

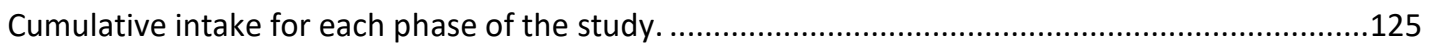

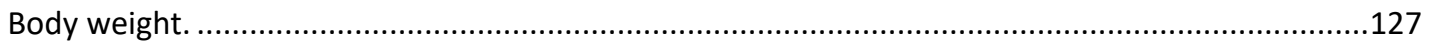

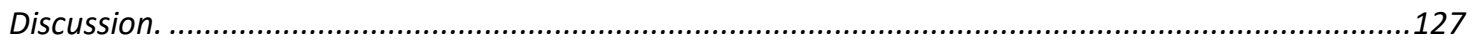

Chapter 5: General Discussion ........................................................................................... 134

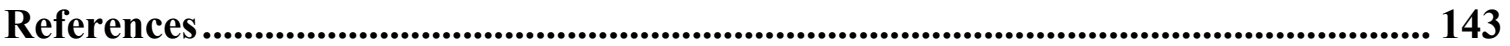




\section{List of Figures}

\section{Chapter 1:}

Figure 1

Chapter 2:

\section{Experiment 1.1}

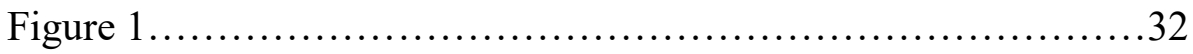

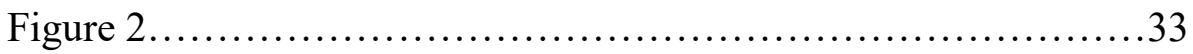

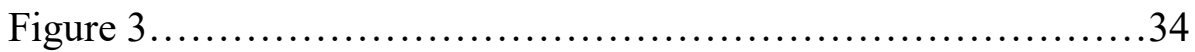

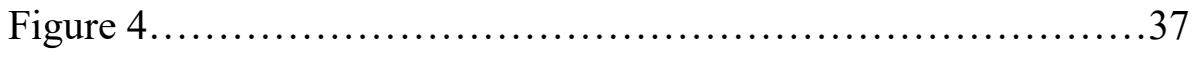

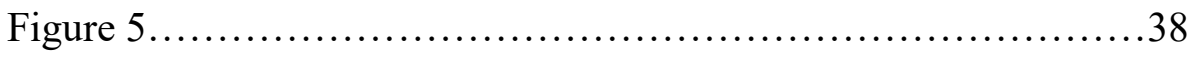

\section{Experiment 1.2}

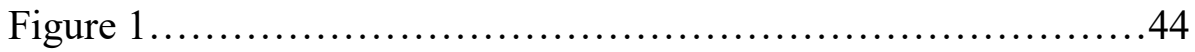

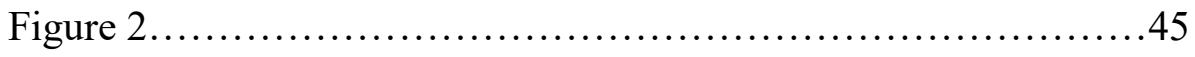

\section{Experiment 1.3.1}

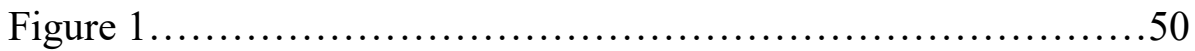

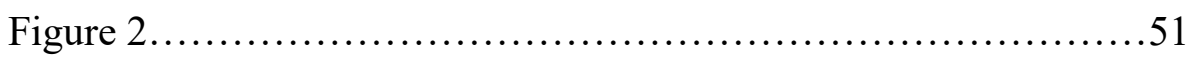

\section{Experiment 1.3.2}

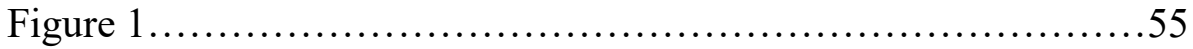

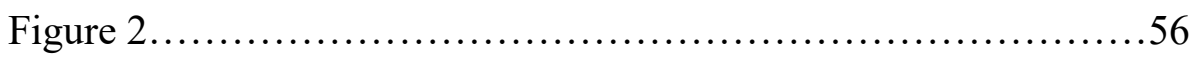

Chapter 3:

Experiment 2.1

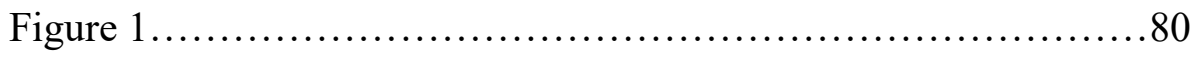

Experiment 2.2

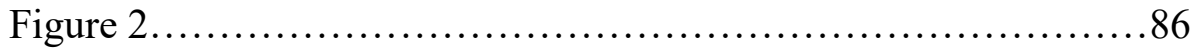

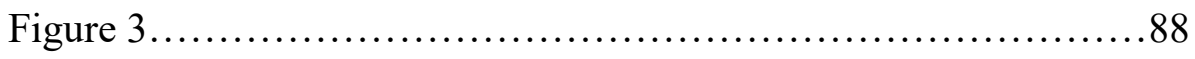

Chapter 4:

\section{Experiment 2.3}

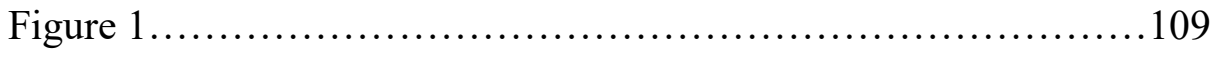

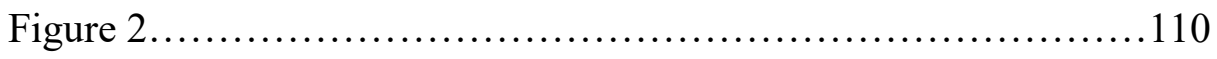

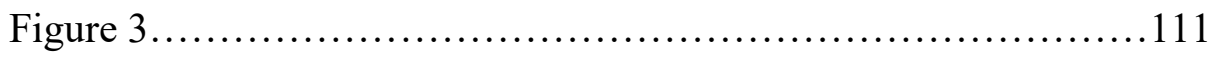

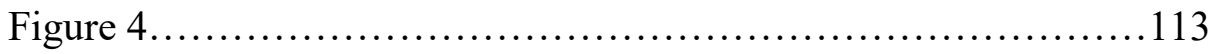




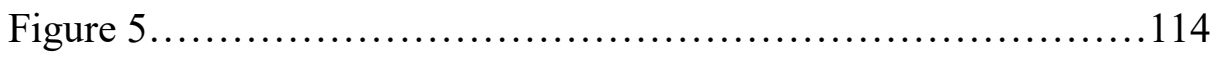

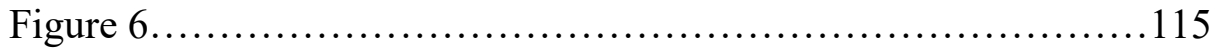

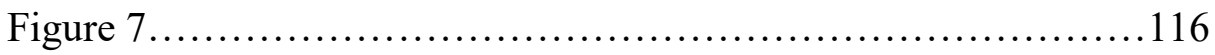

\section{Experiment 2.4}

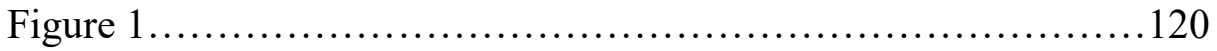

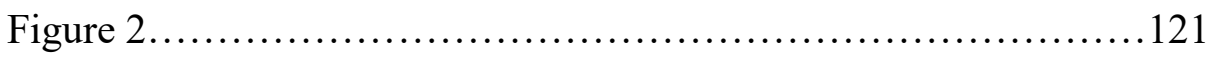

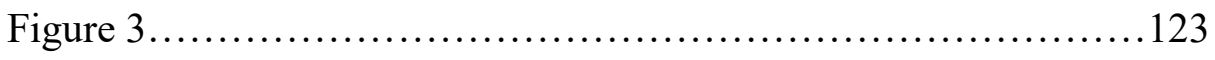

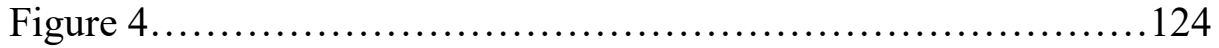

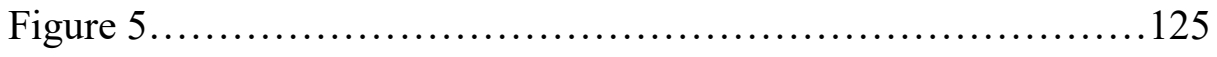

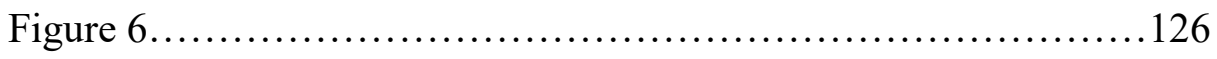

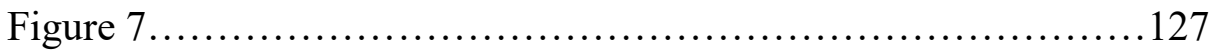




\section{List of Tables}

Chapter 2:

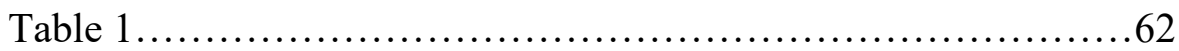

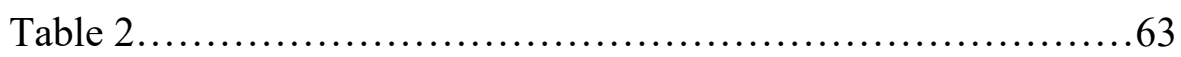

Chapter 3:

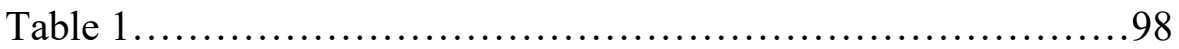




\section{List of Appendices}

Chapter 2:

Appendix A.................................................65

Appendix B.................................................66 


\section{Preface.}

Ghrelin has been studied extensively by many research groups in relation to its regulation of appetite and other metabolic parameters including weight gain and energy expenditure. The rationale for this research stems not only from the apparent rise of obesity and its associated health complications worldwide (Lawrence \& Kopelman, 2004), but also from attempts to bolster appetite under conditions such as anorexia and/or cachexia (Akamizu \& Kangawa, 2011; DeBoer, 2011). The optimal homeostatic profile, wherein a balance between energy consumed and energy expended is maintained over the long-term, is impaired when an individual is overweight or obese. The prevalence of this impairment is thought to be facilitated by an increasingly sedentary lifestyle coupled with an environment in which access to highly palatable food is convenient and affordable (Kopelman, 2000). Consumption of foods high in fat and sugar have been shown to alter neurobiological function and can serve to promote over-indulgence and unhealthy eating habits in a variety of different contexts (Johnson \& Kenny, 2010; Avena et al., 2008). Binge consumption of such foods can also result in cognitive, metabolic and psychological dysfunction in both humans and animals (APA, 2013; Avena et al., 2009; Leibowitz \& Hoebel, 2004; Pelchat et al., 2004; Wang et al., 2004; Jansen et al., 1989).

Ghrelin regulates both homeostatic and reward-related neurocircuits to enhance both appetitive and consummatory aspects of feeding. Its receptor, the growth hormone secretagogue receptor (GHSR), is well-positioned in the central nervous system to play a role in both the homeostatic regulation of feeding, as well as eating for pleasure or reward derived from palatable foods in the absence of caloric need, the latter often referred to in the literature as hedonic feeding (Bello et al., 2009; Berridge et al., 2010; Meye and 
Adan, 2014). In rodents, central ghrelin administration has been shown to enhance the motivation to consume a variety of palatable foods, as measured by progressive ratio (PR) responding (King et al., 2011; Skibicka et al., 2011a; Overduin et al., 2012; St.Onge, Watts \& Abizaid, 2015). In healthy human participants, activity in brain regions involved in hedonic responses to food-related cues were increased (as were hungerratings) following acute administration of intravenous ghrelin (Malik et al., 2008), suggesting that peripheral ghrelin may have significant effects on the central nervous system.

We attempt herein to determine whether GHSR signaling is involved in the development and/or maintenance of a number of food-motivated behaviours exhibited following exposure to either sucrose or fat. To evaluate the role of the GHSR in sucrose reward, we used rats lacking the GHSR and their wild-type (WT) littermates, testing them on a number of conditioning paradigms traditionally used to evaluate the reinforcing properties of drugs that also show efficacy in evaluating food reward, namely PR responding and conditioned place preferences tests (Studies 1.1 and 1.2, Chapter 2). We hypothesized that a lack of GHSR signaling would impair the expression of food reward behaviours in each of the tests conducted. We further hypothesized that GHSRs in the ventral tegmental area (VTA), a key central node in the mesolimbic dopamine (DA) system, would be important for sucrose preference. We tested this hypothesis using mice that harbor a transgene preventing the expression of the GHSR that can be rescued via viral infusion and examined preference for sucrose using a two-bottle choice test before and after rescue of the GHSR (Studies 1.3.1 and 1.3.2, Chapter 2). These studies also served as validation of the method of receptor reinstatement by way of infusing an adeno- 
associated viral (AAV) vector delivering cre-recombinase into the VTA of GHSR null and WT mice and determine the degree of re-expression achieved relative to WT mice.

To examine the role of GHSRs in the binge-like consumption of high-fat diets in mice, we used a limited access to high-fat paradigm (adapted from: Corwin et al., 1998). The original model was generated based on the findings that limiting access to various substances typically enhances their intake upon reintroduction (Wayner \& Fraley, 1972; Pinel \& Huang, 1976), inducing binge-like intake. In Studies 2.1 and 2.2 (Chapter 3), we explored the efficacy of the limited access model to induce binge-like eating in mice and explored the involvement of the ghrelin system using ghrelin-receptor knock-out (GHSR $\mathrm{KO}$ ) and WT mice, respectively. As ghrelin signaling may play a role in bingeing on palatable foods, we hypothesized that mice lacking GHSR would show either a deficit in the development of binge eating or a reduced magnitude of binge-like behavior.

In the final set of experiments, we aimed to determine whether reactivation of GHSR in the VTA would result in an amplification of binge-like feeding on a high-fat diet, hypothesizing that it would enhance bingeing (Study 2.3, Chapter 4). Additionally, we hypothesized that pharmacological antagonism of GHSR in the VTA would reduce high-fat bingeing in mice once it had been established, with an aim to evaluate the therapeutic potential of GHSR antagonism in the context of fat bingeing (Study 2.4, Chapter 4). Overall, the data derived from this series of studies was hypothesized to provide novel insight into whether GHSR signaling is important for both appetitive and consummatory behaviours related to sucrose and fat exposure, and whether the VTA is a key neural substrate for this behaviour. 


\section{Chapter 1. The role of ghrelin in the central regulation of feeding.}

Regulation of feeding behavior in mammals.

Feeding is a necessary component of an organisms' survival and reproductive success, and its regulation by central nervous system (CNS) mechanisms is complex and multifaceted (Schwartz et al., 2001). In mammals, food intake is a regulatory process ensuring that the body receives adequate energy (i.e. glucose) to meet its physiological demands. Homeostatic mechanisms provide the organism with a means of sensing the nutritional content of food and monitoring energy stores, providing feedback to increase or decrease energy expenditure as necessary (Morton, Meek \& Schwartz, 2014).

Meals are terminated when the CNS processes signals that arise from the sensory experience of ingesting calories, but the signals that contribute to the appetitive response and initiation of meals are seemingly more complex (Schwartz \& Zeltzer, 2014). Reduced concentrations of satiety signals, as the time elapsed since the last meal increases, contribute to the induction of hunger to maintain caloric homeostasis (Squire et al., 2008). However, additional variables such as learning, social factors, environmental stimuli, and food palatability are also known to influence ingestive behaviour. Mammalian species have evolved several neural mechanisms to facilitate the acquisition of an abundance of nutritive food sources. In fact, both peripheral and central mechanisms of energy homeostasis culminate in an overall tendency towards anabolic (i.e. energy seeking) rather than catabolic (i.e. energy utilization) processes (Schwartz et al., 2003). This could be the case, in part, due to reward circuitry within the CNS facilitating the response to and reinforcement by food and its associated cues, as well as the consumption of and motivation to obtain palatable food above and beyond that regulated by homeostatic circuitry (e.g. Kelley et al., 2003). In fact, these systems show 
both independent and integrated activity in different contexts and are regulated by many neuropeptides that are synthesized both centrally and peripherally (Abizaid et al., 2009; Fulton, Woodside, \& Shizgal, 2000; Figlewicz \& Sipols, 2010; for review see Saper, Chou \& Elmquist, 2002).

\section{Central nervous system control of feeding.}

The hypothalamus.

Early lesion studies in rats demonstrated the importance of the hypothalamus in regulating food intake (Hetherington, 1941; Hetherington \& Ranson, 1942). Briefly, lateral hypothalamic (LH) lesioned rats exhibited hypophagia (i.e. a reduction in feeding) whereas rats that sustained large lesions to the ventromedial hypothalamus (VMH) displayed both hyperphagia and obesity. Electrical stimulation studies further supported these findings, showing that stimulation of the LH and VMH produced obesity and anorexia, respectively (Coons \& Cruce, 1968; Valenstein et al., 1968). Taken together, this research led to a 'dual center' hypothesis suggesting that the LH was responsible for orexigenic signaling (i.e. stimulating appetite) and that, conversely, the VMH provided anorexigenic signals (Elmquist, Elias \& Saper, 1999). However, this early research lacked the ability to address questions regarding the dynamic ability of these regions, and others, to influence the short-term regulation of meal patterns, the long-term maintenance of body weight, and the influence of motivation on feeding behaviour.

Subsequently, it was found that these discrete hypothalamic nuclei did not serve simply as hunger and satiety centers. Disrupting the connections between the VMH and the paraventricular nucleus of the hypothalamus (PVN) was more effective at stimulating feeding (Gold ,1973), and interruption of the ascending nigrostriatal bundle (as a 
corollary of LH lesions) resulted in other impairments (e.g. motor deficits) that could reduce feeding behaviours artifactually (Stricker \& Verbalis, 1990).

Within the hypothalamus, the arcuate nucleus (ARC) plays an integral role in maintaining a balance between energy intake and expenditure. As the neurons within this region are proximal to fenestrated capillaries, they gain access to circulating factors that may not be able to act directly on other parenchyma (Benoit et al., 2000; Cone et al., 2001). Two distinct neuronal phenotypes were identified in the ARC, subsets of which contain the primary inhibitory neurotransmitter gamma-aminobutyric acid (GABA; Horvath et al., 1997; Hentges et al., 2004), and whose afferent and efferent components are broadly referred to as the melanocortin system. One of these neuronal populations express proopiomelanocortin (POMC) and cocaine-amphetamine related transcript (CART). These neurons produce multiple peptides, including $\alpha$-melanocyte stimulating hormone ( $\alpha-\mathrm{MSH})$ and adrenocorticotropin hormone (ACTH). Upon release of $\alpha-\mathrm{MSH}$, food intake is suppressed and energy expenditure is elevated through activation of downstream melanocortin-3 (MC3R) and melanocortin-4 receptors (MC4R; Cone, 2005). Animals or humans with disrupted POMC signaling or expression have been shown to develop obesity (Spiegelman \& Flier, 2001). The second population expresses neuropeptide Y (NPY) and/or agouti-related peptide (AgRP), which upon activation, antagonize the MC3R and MC4R (Adan et al., 1994) and result in the stimulation of feeding and a reduction of energy expenditure (Cone, 2005). An appropriate balance in the opposing activity of these neuronal subsets ensures well-maintained homeostasis. The anatomical position of these neurons and their co-expression of a variety of receptors 
activated by circulating peptides is important for both short-term and long-term energy balance, rendering them integral to feeding behaviour.

\section{Peptide regulation of food intake in the hypothalamus.}

The hypothalamus is one of a few brain regions classified as circumventricular organs (CVOs), that can access peripherally circulating peptides. The hypothalamus is adjacent to the median eminence (ME), which connects the hypothalamus to the pituitary gland, and contains an extensive vasculature, allowing sampling from the portal system. Interestingly, the metabolic status of an organism can either increase or decrease the ability of circulating factors to gain access to these regions, which can have an impact on food intake and energy status (Schaeffer et al., 2013). Peptide ligands first act on receptors within the ARC (consequently referred to as "first order" neurons) and can have many indirect effects downstream of this region, in alternate hypothalamic nuclei as well as extra-hypothalamic regions.

In early work, Harris and Martin (1984) demonstrated that after a period of food deprivation and reduction in body weight, mature female rats ate larger meals to compensate until their body weight returned to its "set point". Conversely, after force feeding and weight gain, smaller meals were consumed by the animals until the weight was lost. The days required to achieve "recovery" varied, however, these compensatory mechanisms suggested that a regulatory feedback system may permit peripheral tissues to relay information to the CNS, indicating when to obtain more nutrients, or conversely, when to stop.

Leptin, the product of the $o b$ gene, is one of the numerous peptides known to regulate feeding via actions in the ARC (Zhang et al., 1994). Leptin is derived from adipose tissue, serving to signify adequate, and potentially an overabundance of, energy 
stores. Synthesized by white adipose tissue (WAT) in the periphery and capable of crossing the blood-brain barrier (Banks et al., 1996), leptin can act directly on its receptors expressed on POMC/CART neurons found in the ARC (Elmquist et al., 1998; Elias et al., 2000), which project to the LH to inhibit feeding, while suppressing signaling from neighboring NPY neurons to the LH (Elias et al., 1999). Leptin receptor signaling within this neuronal population is critical for normal body weight homeostasis (Balthasar et al., 2004). Mice that lack the gene coding for leptin $(o b / o b)$ become hyperphagic and obese, whereas direct infusion of leptin causes the knock-out mice to reduce their food intake (Zhang et al., 1994).

Conversely, several orexigenic peptides have been implicated in the facilitation of appetite, in both the short- and long-term. For example, administration of AgRP and NPY have both been shown to rapidly increase food-seeking behaviours, including instrumental responding for food pellets (Tuebner et al., 2012; Aponte et al., 2011). Administration of ghrelin, both centrally and peripherally, also induces such behaviours (Abizaid et al., 2006; King et al., 2011), and is known to act directly via its receptors on both AgRP and NPY neurons in the ARC (Nakazato et al., 2001; Cowley et al., 2003) to promote feeding and appetite (see below for additional details pertaining to ghrelin).

The midbrain dopamine system.

The necessity of dopamine (DA) signaling for feeding is illustrated by the fact that mice unable to produce DA die of starvation (Saper, Chow \& Elmquist, 2002), and additional studies show that disrupting DA pathways can generate a similar phenotype, though not as certainly fatal (Ungerstedt, 1971). Multiple DA systems in the brain could serve to facilitate feeding, however, as food is a natural reinforcer required for survival, it is posited that the mesocorticolimbic dopamine system is likely to be the primary system 
driving feeding behaviour. This could be particularly true as it relates to consumption of palatable foods.

Experiencing pleasure or reward from eating is thought to be regulated by the mesolimbic DA system. This primitive reward circuit in the brain consists of DA neurons that reside in the VTA and contain efferent projections to the nucleus accumbens (NAcc). VTA DA neurons also contain projections to additional nuclei including the amygdala, prefrontal cortex (PFC), and hippocampus (Kelley \& Berridge, 2002). The role of DA in eliciting and/or maintaining food reward behaviours is continuously being investigated but is yet to be fully understood.

In recent years, many studies have highlighted that food and drug reward have somewhat overlapping neural substrates. The overconsumption of highly palatable food leads to behavioural and physiological changes similar to those seen in individuals addicted to drugs of abuse (e.g. Johnson \& Kenny, 2010). Such analogous findings, including a lack of control and altered neuroplasticity, suggest that the mesolimbic dopamine circuitry is highly involved in "food addiction", for which scientific evidence is rapidly accumulating (Volkow \& Wise, 2005). In fact, without functionality of this hedonic reward pathway, inherently tasty foods can rapidly lose their appeal (Berridge, 2009). Interestingly, chronic food deprivation and diet-induced obesity induce similar changes in DA systems (Pothos \& Sulzer, 1998), circumstances which both lead to enhanced motivation to obtain food reward.

As suggested by Berridge (2009), the 'liking' of a food is triggered by the action of endogenous opioid release in the NAcc upon consumption. However, the subjective pleasure experienced must be translated into a 'wanting' or motivation to obtain that food 
in the future to generate an influence on food intake, an effect that is highly dependent on the dopaminergic system. A study by Barbano et al. (2009) demonstrated this neurochemical dissociation by administering naloxone or flupenthixol to rats trained to either traverse a straight alley runway or bar press to obtain food rewards. Blockade of DA receptors affected the threshold of the cost/benefit ratio, and rats were less likely to bar press for palatable food pellets. Blockade with naloxone on the other hand, resulted in a decreased consumption of palatable foods not associated with the effort required to obtain it (Barbano et al., 2009).

Incentive salience can be defined as the ability of a reward or cues that predict reward (Schultz, 1998) to elicit motivated behaviours (Berridge \& Robinson, 1998; Everitt \& Robbins, 2005; Salamone \& Correa, 2002). Once associated with 'liking' of a reward, these predictive cues can come to trigger cravings, or 'wanting'. Such cues can become so potently motivating that simply thinking about the sight, taste, and smell can trigger a craving in humans (Pelchat et al., 2004). The neurochemical basis of such motivation appears to be the efflux of DA in the NAcc upon activation of DA cell bodies in the VTA. The amount of NAcc DA is positively correlated with the physical effort an animal is willing to exert during an instrumental task to gain a food reward, and DA depletion within the NAcc reduces operant responding for food (Salamone et al., 1991; Salamone \& Correa, 2002). A strong case has been made for the contribution of DA to food reward, although it is widely accepted that the relationship between DA systems and reward are quite complex (Saper, Chou \& Elmquist, 2002). Interestingly, melanocortins (e.g. $\alpha$-MSH) have recently been shown to act directly in the VTA to modulate food reward behaviours such as operant responding (Shanmugarajah et al., 2017), 
demonstrating the importance of crosstalk between hypothalamic and midbrain dopaminergic circuitries in driving the motivation to consume palatable reinforcers.

\section{Ghrelin.}

Ghrelin is a 28 -amino acid peptide that is produced primarily by endocrine mucosal cells in the stomach (Kojima et al., 1999; Date et al., 2000). The active form of ghrelin (acyl-ghrelin) is derived from its precursor preproghrelin (117 amino acids) and is post-translationally modified by the addition of an octanoyl group at the third serine residue (Gutierrez et al., 2008; Yang et al., 2008). The octanoylation process is performed by ghrelin-O-acetyltransferase (GOAT) and is required for the binding of ghrelin to its receptor (see Figure 1). Ghrelin lacking the octanoyl group (or deacylated by proteases) is referred to as des-acyl ghrelin (DAG), and is the most abundant form in circulation (Kojima et al., 2005). DAG was initially thought to be inactive (Satou et al., 2011), though recent findings suggest that it may play a role in the stress response and anxietyrelated behaviours and may act in the ARC in a GHSR-independent manner (Stark et al., 2016; Fernandez et al., 2016). The alternate gene product derived from the ghrelin gene is obestatin (23 amino acids; Zhang et al., 2005), which has been shown to have effects opposite to ghrelin in relation to feeding and growth hormone $(\mathrm{GH})$ release (Zizzari, et al., 2007; Inhoff et al., 2008). The transcriptional regulators influencing the ratio of ghrelin to obestatin production remain unknown, and more research is required to understand the relative importance of both des-acyl ghrelin and obestatin signaling.

Ghrelin has its effects on the brain via binding to the growth-hormone secretagogue receptor of the 1A subtype (GHSR; Howard et al., 1996). The GHSR is a member of the 7-transmembrane metabotropic G-protein-coupled receptor (GPCR) family, whose activity results in the activation of multiple intracellular signaling 
pathways. In addition to its ligand-dependent activity, a high-level of constitutive activity has been observed for this receptor (Holst et al., 2003). Diffuse regions of the rat, mouse, and human brain demonstrate appreciable levels of GHSR expression as detected by in situ hybridization (Guan et al., 1997; Zigman et al., 2006; Gnanapavan et al., 2002), which is not surprising given the wide array of physiological processes for which ghrelin is important.

The most well characterized functions of ghrelin to date include the stimulation of growth hormone $(\mathrm{GH})$ release and the regulation of food intake and energy balance, in both the short- and long-term (Cummings, 2006). Many of these physiological functions are mediated centrally, as ghrelin can gain access to the brain either at CVOs with fenestrated capillaries (e.g. Schaeffer et al., 2013) or via active transport mechanisms (Banks et al., 2002).

Ghrelin has been shown to stimulate the release of GH when administered to both rodents and humans (Date et al., 2000; Kojima et al., 1999; Takaya et al., 2000). It is also the only known orexigenic peptide that stimulates feeding when given peripherally (Tschop et al. 2000; Hosoda, Kojima \& Kangawa, 2006; Druce et al., 2005). Ghrelin increases both food intake and body weight gain when administered systemically, via acute or chronic injections in rats (Lawrence et al., 2002; Tschop et al., 2000; Wren et al., 2000; Wren et al., 2001).

Ghrelin plays a role in meal initiation by peaking just prior to a meal in schedule fed humans and rodents (Cummings et al., 2001, Drazen et al., 2006). Plasma ghrelin also rapidly decreases post-prandially in a manner related to both the caloric load and the nutritional composition of the food consumed, with fat having the most modest effect on 
suppression following its consumption (Foster-Schubert et al., 2008; Callahan et al., 2004; Overduin et al., 2005). During a state of negative energy balance, ghrelin is elevated and acts on GHSRs present on NPY/AgRP neurons in the ARC to inhibit the activity of POMC neurons, and ultimately the downstream melanocortin system (for review see Horvath et al., 2001). Interestingly, ghrelin lacks the ability to induce feeding on regular chow in mice that lack both NPY and AgRP expression (Chen et al., 2004). This increase in ghrelin during negative energy balance also results in greater accumulation of body fat stores and a concomitant reduction in physical activity, leading to increased weight gain and reduced basal metabolic rate (Tschop, Smiley and Heiman, 2000). During times of energy abundance, plasma ghrelin is decreased, and this often remains the case in long-term conditions such as obesity (Tschop et al., 2001). The effects of ghrelin on adiposity appear to be independent of its stimulation of GH and are thought to involve neural circuits that control food intake, energy expenditure and reward (Cowley et al., 2003).

Ghrelin and dopamine in the regulation of food reward. As ghrelin can act within the hypothalamus to regulate homeostatic feeding, as well as on VTA neurons to enhance the motivation to obtain foods that are highly palatable, it may play a unique role in regulating the behavioural and physiological responses to food rewards. In support of its role beyond homeostatic feeding (i.e. consumption in the absence of caloric need) ghrelin maintains the ability stimulate food intake in animals that are satiated, when given either acutely or chronically (Tschop et al., 2000; Nakazato et al., 2001; King et al., 2011). Therefore, ghrelin may act on sites alternate to the ARC to enhance the salience or palatability of food sources. 
Early studies demonstrated that ghrelin selectively increased the consumption of foods high in caloric value, particularly high-fat foods. When rats were given acute ICV injections of ghrelin and given a choice between two types of food, they chose fat most frequently (Shimbara et al., 2004). The same effects were also seen when rats were given free choice between high protein, high carbohydrate or high-fat diet following chronic administration of ghrelin into the VTA (King et al., 2011). Considering this evidence, perhaps ghrelin acts on mesolimbic DA circuitry not to maintain energy balance, but to increase motivation and attention directed towards tasty foods high in caloric content. In fact, a study in which AgRP signaling was ablated in mice demonstrated the ability of a high-fat high-sugar diet to remain capable of inducing overeating (Denis et al., 2015), an effect which may be mediated in part by ghrelin.

In support of this hypothesis, the presence of GHSR message has been reported in VTA, an area known to be important for reward and reinforcement (Guan et al., 1997; Zigman et al., 2006). Ghrelin binds to the VTA, is co-localized with DA neurons (Abizaid et al., 2006), and when administered acutely it produces a robust feeding response (Naleid et al., 2005) with a similar magnitude as would be expected following a sustained period without food (Abizaid et al., 2006). Conversely, blocking ghrelin in the VTA using a selective GHSR antagonist, BIM28163, blunted peripherally induced ghrelin food intake, reflecting less of an interest in food (Abizaid et al., 2006). More recent work from our lab has shown that rats with a truncated GHSR gene $\left(\mathrm{GHSR}^{\mathrm{m} 1 / \mathrm{Mcwi}}\right)$ demonstrate reduced food anticipatory activity as well as reduced "dessert" consumption while satiated, which could reflect disrupted reward system functioning (MacKay et al., 2016). 
Systemic ghrelin administration has been shown to increase extracellular DA in the NAcc shell region as measured by in vivo microdialysis (Quarta et al., 2009). These results are in accordance with earlier work, wherein ghrelin administered directly into the VTA caused a rapid increase in glutamatergic inputs onto VTA DAergic neurons (Abizaid et al., 2006), a mechanism by which the extracellular concentration of DA in the NAcc could be increased (Jerlhag et al., 2006). Results of the latter study also showed an increase in locomotor activity in ghrelin treated animals, an effect consistent with heightened DA transmission.

Conditioned place preference (CPP) is a classical conditioning paradigm that is used to evaluate the preference for an environment that has been paired with a particular reward. Rats that are pretreated with systemic ghrelin display an augmented place preference for an environment paired with a sub-threshold dose of cocaine compared to rats that received saline pretreatment (Davis et al. 2007), which may reflect the ability of ghrelin to enhance the effects of a drug stimulus. Further, pretreatment with ghrelin also increased the preference for an environment paired with HFD but failed to do so in GHSR KO mice (Perello et al., 2010). These mice consumed less food, stored fewer calories, primarily utilized fat as energy substrate, and had lower adiposity than WT control mice (Zigman et al., 2005), although it is unknown whether the direct mediation of these outputs involves ghrelin/DA interactions.

Ghrelin has also been shown to affect the hedonics of feeding in humans. When injected peripherally with physiologically relevant doses of ghrelin, feeding responses were increased, in addition to self-reports of hunger and enhanced imagery of preferred foods (Wren at al., 2001; Cummings et al., 2004). Ghrelin also increases brain activity in 
areas within the mesolimbic dopamine system, such as the NAcc and the amygdala, as well as the perceptions of hunger (Malik et al., 2008). Taken together, results from human studies implicate ghrelin in the enhancement of the palatability of food that is offered (Druce et al., 2006).

Ghrelin and the consumption of sweets.

As mentioned above, palatable food intake can be motivated by pleasure, rather than caloric necessity. Foods high in sugar, or sugar-sweetened beverages, have the capacity to activate the mesolimbic DA and opioid systems in the brain, facilitating future intake (Sclafani, 2004; Berridge, 1996). Overconsumption of sweets can also be facilitated by ghrelin signaling through its putative action on these same systems. Many of the studies examining the role of ghrelin and/or the GHSR on palatable food intake have used sucrose as a reinforcer and have found that ghrelin enhances the motivation for sucrose as measured by PR responding (Skibicka et al., 2011a; Skibicka et al., 2012a,b). Peripheral injection of ghrelin also enhances the preference for sweets, and mice lacking the GHSR do not show a ghrelin induced preference for a saccharin solution, as compared to WT mice (Disse et al., 2010; Landgren et al., 2011). Peripheral injections of ghrelin also evoked greater responding for a 5\% sucrose solution in rats, but interestingly, did not enhance the palatability of a liquid test food (60\% carbohydrates, $20 \%$ fat and $20 \%$ protein), as measured by lick patterns (Overduin et al., 2012). In the same study, administration of a DA D1 receptor antagonist blocked the ghrelin stimulated pressing for sucrose solution (Overduin et al., 2012). These studies highlight the importance of ghrelin in the appetitive and consummatory responses to sweets and suggest that the VTA may be an important region driving such responses. 


\section{Binge eating.}

Abundant and readily available highly-palatable food sources that act upon CNS reward circuitry may serve to entice individuals to eat unhealthily, driven by the hedonic properties of food rather than its nutritional properties. Evolutionarily, such complementary drives would be beneficial, as seeking out high calorie and pleasant tasting food would promote survival. Such a mechanism would also likely steer an animal away from poisonous foods. A dysregulation of this system, with failure to adapt to the ever-increasing obesogenic environment poses some important health risks and can often result in binge eating.

Binge eating is defined by the consumption of an excessive amount of food in a short time frame and is often associated with a sense of loss of control over the ability to cease eating once nutritional needs have been met (APA, 2013). Binge eating, therefore, reflects a form of hedonic eating that is not motivated by the necessity for calories or specific macronutrients, and is likely to be regulated in part by mesolimbic reward circuitry (Davis et al., 2009; for a review, see Bello and Hajnal, 2010). In support of this view, it is most often highly-palatable and energy- dense foods that are consumed during a binge episode (Kales, 1990; Elmore and DiCastro, 1991), and consumption of such foods are known to influence the short- and long-term plasticity of both homeostatic and brain reward circuitry (Bello et al., 2009; Johnson and Kenny, 2010). The precise mechanisms underlying the eating behavior seen in individuals that binge eat remain poorly understood. Identifying the factors that contribute to binge eating in both normal weight and obese binge eating disorder (BED) models, components of which may be dissociable, will aid in the understanding of the disorder and ideally improve treatment options for those that binge eat. This need appears more urgent for those who binge eat 
and are also obese, as these individuals tend to suffer more severe physical and mental health consequences (da Luz et al., 2018).

Binge eating can occur in isolation, but also occurs in the context of other eating disorders, such as anorexia (AN) and bulimia nervosa $(\mathrm{BN})$, making it the most common form of disordered eating. As of 2013, BED was included in the Diagnostic and Statistical Manual of Mental Disorders as a disorder that can cause both psychological and physiological disturbances (DSM-V; American Psychiatric Association, 2013). BED is the most common eating disorder for which treatment is sought, particularly in the United States (Hudson et al., 2007). The development of BED is common in both adolescent and adult populations of both sexes globally, rendering it highly prevalent across cultures and geographical regions (Kessler et al., 2013). In addition, while the majority of problematic binge eating develops between 10 and 20 years of age, onset of the behavior can occur well into the senior years (Hudson et al., 2007).

In an attempt to elucidate the predictors, consequences, and underlying physiological mechanisms related to binge eating behavior, a number of animal models of binge eating have been developed (Bake et al., 2014; Corwin et al., 1998; Bello et al., 2009; Boggiano et al., 2007; Colantuoni et al., 2002; Berner et al., 2008; Lardeux et al., 2013; Parylak et al., 2008). The limited access model, first demonstrated in rats by Corwin et al. (1998), is used to elicit binge-like consumption and patterns of fat intake that emulate components of eating behaviors seen in humans with BED. Restricting access (voluntarily or involuntarily) is thought to partially underlie the repeated failures of dieters who attempt to self-restrict intake of palatable foods. In this model, rats placed under time restricted and sporadic access to fat demonstrate a binge-compensate pattern 
of feeding, consuming a greater number of calories on access days, and fewer calories on non-access days, relative to the control group who do not receive fat (Corwin et al., 1998). The primary benefit of this model is that it fosters the development of binge-like patterns of intake without requiring caloric restriction or stress, both of which can impart a number of physiological confounds that substantially influence feeding behaviors. While this model does not accelerate the development of obesity in all studies, it can alter patterns of intake and dietary composition, which may be reflected in changes in endocrine function and CNS activation.

The role of ghrelin in the development or maintenance of binge eating remains unclear. Elevated ghrelin levels were shown to be present in individuals with the bingepurge form of anorexia nervosa (Tanaka et al., 2003a,b; 2004). Another early study implicated the presence of the Leu72Met ghrelin gene variant in the development of BED in a small cohort of human subjects (Monteleone et al., 2007), but not in those with anorexia nervosa or bulimia nervosa (Monteleone et al., 2006). Additional studies have reported that plasma ghrelin is reduced in obese-BED individuals (Geliebter et al., 2005; Monteleone, 2005; Troisi et al., 2005), which likely reflects a long-term adaptation resulting in the down-regulation of ghrelin secretion as a consequence of excessive body weight gain and/or food consumption (Atalyer et al., 2012). Interestingly, the postprandial suppression of ghrelin is attenuated in obese-BED populations (Geliebter et al., 2008), which may reduce the satiating effect of a meal and may contribute to over-eating in the short term. It is currently unknown whether ghrelin signaling in normal-weight populations of humans or rodents is required for the development of binge eating on high-fat foods. 
Using genetic knock-out and adeno-associated viral delivery of cre to evaluate the necessity of GHSR in food reward.

Establishing the importance of the GHSR in certain behaviours can be facilitated by an evaluation of behavioural responding in its absence, through use of a genetic knock-out model. If the behaviour is exaggerated or inhibited without the presence of the GHSR, it is likely that it plays an important role in said behaviour (e.g. sucrose preference or high fat bingeing). As a number of genetic models were available to us, we used such methods to assess the role of GHSR in food-motivated behaviours. To determine whether the VTA might be an important site of GHSR function in regulating these behaviours, we used an adeno-associated viral (AAV) vector approach combined with cre-recombinase technology to "rescue" the GHSR in this region and examine subsequent behaviours.

Adeno-associated viruses are non-replicating parvoviruses with an icosahedral capsid, about $25 \mathrm{~nm}$ in diameter, and are capable of packaging up to $\sim 4.7 \mathrm{~kb}$ of genetic material (Bowles et al., 2006). Stable AAV mediated gene expression has been successful in the CNS in a number of animal models, however, tropism for specific cell types has been shown to be influenced by the serotype of the AAV used (Burger et al., 2004; Cearley and Wolfe, 2006). Previous studies have demonstrated that viruses harboring an AAV9 serotype are effective at transducing neurons in the mouse in vivo (Vincent et al., 2014).

In the current studies, we combined this AAV technology with a transgenic mouse line harboring a transcriptional blocking cassette upstream of the GHSR promoter region (Zigman et al., 2005; see Chapter 2, Appendix A), which prevents transcription of the GHSR gene (GHSR null). The transcriptional blocking cassette is flanked by two lox- 
P sites, allowing for its excision upon delivery of cre-recombinase, which can be successfully delivered using AAV (Ahmed et al., 2004). Upon delivery of cre, whose expression is driven in neurons exclusively by the human synapsin promoter (SYN; Glover et al., 2002), reinstatement of the receptor occurs in the region of choice. This can also be achieved using cre-driver lines of transgenic mice that express cre in specific subtypes of neurons (e.g. tyrosine hydroxylase (TH) neurons; Chuang et al., 2011). However, in the current studies our goal was to establish reinstatement in neurons within the VTA rather than throughout the entire brain.

Objectives of the current thesis.

In the current thesis, the overarching hypothesis was that the GHSR is required for the full expression of food-reward behaviours using both sugar and fat reinforcers, in models assessing the appetitive and consummatory aspects of feeding. In Chapter 2, we hypothesized that a lack of GHSR in rats would hinder responding for food reinforcers on a PR schedule of operant responding, during which rats have to work increasingly hard to obtain a variety of food pellets. We then aimed to determine whether GHSR is necessary to form a CPP for sucrose and examined the necessity of GHSR signaling in the VTA on sucrose preference in mice with a two-bottle choice test, using AAV-CRE mediated technology.

In Chapter 3, our objective was to examine the efficacy of the limited access to high-fat diet model to induce binge-like eating in mice and further explore the role of the GHSR signaling in its development and/or maintenance. We hypothesized that providing mice with restricted and intermittent access to HFD would cause binge consumption of the HFD, and that mice lacking the GHSR will show hindered development of binge eating in this context. 
In Chapter 4, we used both genetic and pharmacological tools to determine whether cre-mediated reinstatement of GHSR transcription in the VTA in mice lacking GHSR from birth was sufficient to rescue high-fat bingeing behaviours. Our hypothesis was that GHSR null mice would initially show a reduced preference for high-fat diet under limited access, but that upon re-expression of GHSR in the VTA binge behaviours would be comparable between genotypes. To determine whether blockade of GHSR in the VTA was able to reduce binge-like feeding on high-fat, we pharmacologically antagonized the GHSR in this region once binge eating had been established. As the VTA is a key site within which ghrelin and DA interact to influence food reward, we hypothesized that mice receiving the GHSR antagonist chronically into the VTA would show reduced binge-eating, potentially providing a therapeutic avenue to pursue for the cessation of binge eating. 


\section{Figure 1.}

Ghrelin peptide sequences and depiction of octanoylation by ghrelin-O-acetyltransferase (GOAT) and subsequent binding to the GHSR-1a.

\section{DESACYL-GHRELIN}

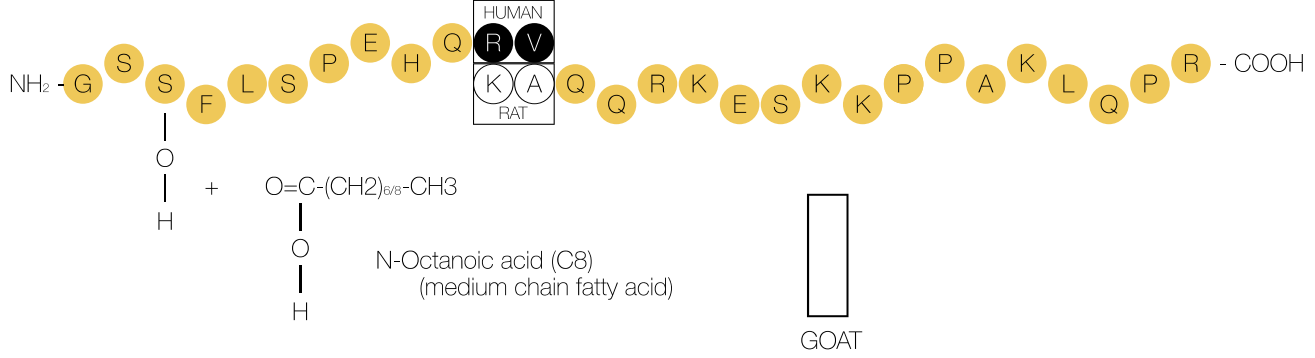

ACYL-GHRELIN (active)

Human M.W. $=3.370,91$ Dalton
Rodent M.W.=3.314,84 Dalton

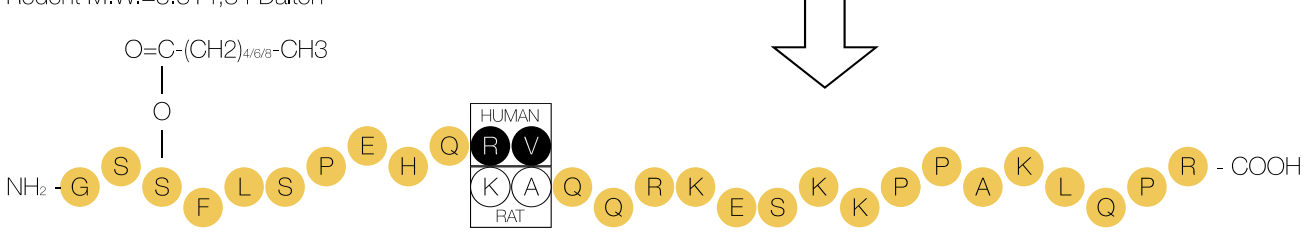

Arg11-Val12 in human replaced with Lys11-Ala12 in rodents (rat/mouse)
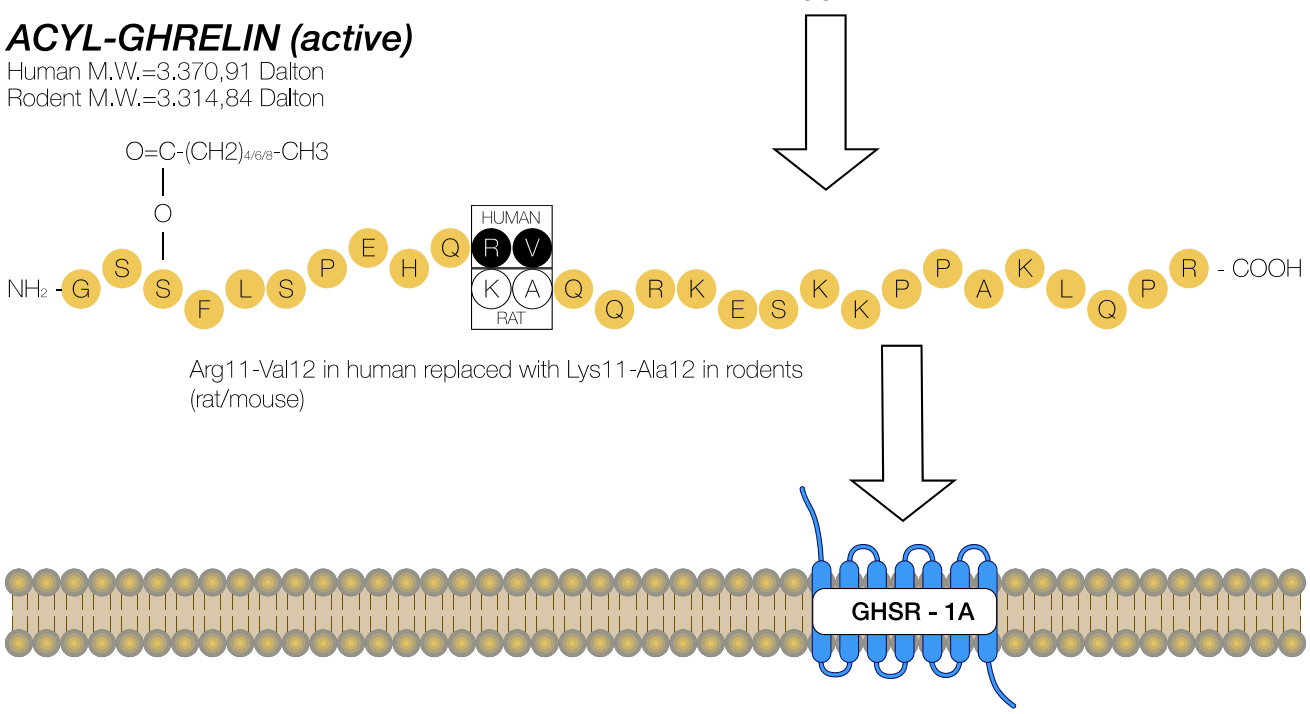


\section{Chapter 2: Rats and mice without functional GHSR exhibit reduced motivation and attenuated preference for sucrose reward, the latter being augmented by rescue of the GHSR in the VTA.}

\section{Introduction.}

The overconsumption of palatable foods, typically characterized by their sugar and/or fat content (Drewnowski et al., 1989), can lead to a number of health problems, including, but not limited to obesity. Due to their rewarding properties, these types of foods have the capacity to elicit consumption in the absence of energetic requirements, which can result in the consumption of calories beyond those required to maintain physiological function. This results in the subsequent storage of these excess calories, rather than their utilization for fuel. In evolutionary terms, having an abundance of energy dense foods available with very little effort required to obtain them, is a relatively new phenomenon. Sweet foods are often overconsumed, and recent evidence has linked the gut derived hormone ghrelin to the appetite for sweet foods (Skibicka et al., 2011a; Skibicka et al., 2012a,b; Disse et al., 2010; Landgren et al., 2011; Overduin et al., 2012).

Ghrelin is a 28-amino acid peptide synthesized mainly in the gut that acts on the growth hormone secretagogue receptor 1-a (GHSR) (Kojima et al., 1999) and is an endogenous regulator of energy homeostasis, acting mainly in the arcuate nucleus (ARC) of the hypothalamus (Cowley et al., 2003). It is also the only known circulating peptide hormone that stimulates feeding, making it "orexigenic", or appetite stimulating (Tschop et al., 2000; Druce et al., 2005). Much of the work conducted on ghrelin in terms of ensuring sufficient caloric intake has involved regions of the brain such as the hypothalamus and the brain stem. However, the GHSR is abundant in other regions of the brain heavily involved in reward and motivation, such as the ventral tegmental area 
(VTA) (Guan et al., 1996; Zigman et al.,2006; Abizaid et al., 2006). The majority of recent findings converge to illustrate the importance of ghrelin in regulating various appetitive and consummatory responses to palatable food and drugs, primarily by way of interaction with the mesocorticolimbic dopamine (DA) system (Wellman et al., 2005; Jerlhag et al., 2009; Jerlhag et al., 2007; Naleid et al., 2005; Abizaid et al., 2006).

In terms of sugar consumption, a number of studies have demonstrated that signaling via the GHSR is required to enhance the intake of sweets, both when an instrumental response is required to obtain sucrose (Skibicka et al., 2011; Skibicka et al., 2012), as well as when the sweet stimuli has no caloric value, as in the case of saccharin (Disse et al., 2010). The latter study is particularly interesting, as access to saccharin was given to both sated and hungry mice and peripheral ghrelin injections stimulated the intake of the sweetened solution, even in the absence of hunger. These results suggest that ghrelin's influence on ingestive behaviour is not limited to purely homeostatic mechanisms. High carbohydrate meals result in a large early post-prandial reduction in circulating ghrelin levels, however 3 hours after consumption of carbohydrates, ghrelin levels increase above those seen in the pre-prandial state (Foster-Schubert et al., 2008). This second rise in ghrelin levels may be associated with an excess intake of carbohydrates at the next meal or snack, hence perpetuating a pattern of increased carbohydrate intake.

In addition to changing their ingestive behaviour, ghrelin treated rats and mice also reliably increase the amount of effort they are willing to exert to obtain palatable food rewards on a progressive ratio (PR) schedule of reinforcement (Landgren et al., 2011), whereas blockade of ghrelin signaling both genetically (GHSR-KO) and 
pharmacologically, reduces the amount of effort expended (King et al., 2011). Previous studies in our lab have shown that ghrelin increases motivation for chocolate-flavoured pellets (King et al., 2011) and that relapse to food-seeking for high-fat chocolate pellets occurs more rapidly in rats administered ghrelin (St.Onge et al., 2015). In addition, we have previously demonstrated that delivery of ghrelin into the VTA enhances the preference for a high-fat diet when presented simultaneously with carbohydrates and protein, whereas GHSR antagonism blocks this preference (King et al., 2011). Many sensory properties of a foodstuff can influence its palatability, for example, in the King et al. (2011) study, the carbohydrate diet was fairly hard, whereas the high fat diet was soft and buttery. It is possible that ghrelin differentially affects the motivation for food reward not only depending on its macronutrient content, but also on its flavour or consistency.

In the first experiment, we utilized a GHSR-KO rat model backcrossed onto a Fawn-Hooded Hypertensive (FHH) strain (GHSR ${ }^{\mathrm{m} 1 / \mathrm{Mcwi}}$ rats), to examine the role of the GHSR on the motivation to obtain pellets of varied caloric value and putative palatability. It is possible that ghrelin differentially affects the motivation for food reward depending on the type of reinforcer being offered, therefore we used a paradigm in which we could alter the content of the reinforcers without significantly altering the sensory properties of each offering. Based on the literature, we hypothesized that $\mathrm{GHSR}^{\mathrm{m} 1 / \mathrm{Mcwi}}$ rats would work less for food reinforcers relative to WT rats, and that we may see larger differences between the genotypes on the more palatable chocolate flavoured, sucrose, and high-fat chocolate flavoured pellets.

To elaborate on the results of experiment 1.1, we used a different approach to determine the rewarding value of food by investigating whether a lack of GHSR 
signaling in rats would also prevent the formation of a conditioned place preference (CPP) for a sucrose reward. Unlike the PR paradigm, CPP is a behavioural test that enables the assessment of the reinforcing properties of an unconditioned stimulus (UCS; e.g.s. food or drugs) in the absence of the stimulus itself. Repeated pairing of the UCS (i.e. the sucrose) with a distinct contextual environment (the CS) results in a learned association between the palatable food and the contextual environment with which it was paired. The context then becomes secondarily reinforcing, and this typically manifests as a voluntary preference of the mouse/rat to spend more time in the side of the apparatus that was paired with the most reinforcing stimulus. Testing in the absence of the food reward allows the analysis to be devoid of any concurrent physiological influence of the food (or drug) itself on behaviour. Given the evidence supporting a role for ghrelin and/or GHSR signaling in reward derived from sucrose, we hypothesized that rats lacking GHSR would show an attenuated CPP for sucrose pellets relative to WT rats.

Previous work has shown that GHSR null mice show attenuated CPP for and consumption of a high-fat diet, and that cre-recombinase (CRE) mediated rescue of the GHSR in central tyrosine hydroxylase $(\mathrm{TH})$ producing neurons restores food reward behaviours in stressed mice (Chuang et al., 2011). However, in these experiments, GHSR was restored in all $\mathrm{TH}$ expressing cells throughout the brain. The role of liganddependent GHSR activation within the VTA specifically on food reward behavior has been demonstrated for a number of behaviours including (but not limited to) CPP, sucrose preference (Landgren et al., 2011), and PR responding for sucrose (Landgren et al., 2011). 
In the final study described here, our main objective was to reinstate transcription of GHSR exclusively in the VTA using a neuron-specific adeno-associated viral (AAV) approach, an effective mode of stable gene transfer in neurons in the central nervous system (CNS) (for review see Samulski and Muzyczka et al., 2014; Murlidharan et al., 2014). Given previous data, we hypothesized that GHSR null mice would show a reduced preference for the sucrose solution, but that upon reinstatement of GHSR in the VTA, these mice would increase their preference for sucrose. If supported, this would illustrate the importance of GHSR in the VTA for sucrose reward, and potentially reward derived from other palatable foods.

\section{Experiment 1.1. Hungry GHSR ${ }^{\mathrm{m} 1 / \mathrm{Mcwi}}$ rats are less motivated to obtain sucrose pellets under a progressive ratio schedule of responding.}

\section{Materials and Methods.}

Animals.

Adult male GHSR ${ }^{\mathrm{m} 1 / \mathrm{Mcwi}}(n=10)$ and WT $(n=10)$ rats $(\sim 96$ days old; N=20; $290-$ $350 \mathrm{~g}$ ) were used in this study. The mutant rats were generated by Transposagen pharmaceuticals (Lexington, KY) via $N$-ethyl- $N$-nitrosourea (ENU) induced mutations, followed by backcrossing onto a Fawn Hooded Hypertensive (FHH) background strain. The WT FHH strain was originally obtained from the University of Wisconsin. The rats were bred within our in-house colony and maintained at the Carleton University animal viviarium. Rats were reared in pairs and individually housed prior to initiation of the experiment in transparent Plexiglass cages (48cm X 26cm X 20cm). Rats were maintained on a reverse 12-hour light dark cycle (lights off at 08:00) in a temperature and humidity-controlled environment $\left(22^{\circ} \mathrm{C}\right.$ and $45-55 \%$, respectively), and had ad libitum access to water and food, except where stated otherwise. All experimental procedures for 
this and experiments 1.2, 1.3.1, and 1.3.2 complied with the Canadian Council on Animal Care (CCAC) guidelines and were approved by the Animal Care Committee at Carleton University.

\section{Caloric restriction.}

Rats had ad libitum access to standard chow for one week in order to establish baseline body weight and average daily food intake. They were then calorically restricted to $50 \%$ of their average daily caloric intake until they reached $85 \%$ of their baseline body weight. Once their target weight was reached, amount of standard chow offered was titrated to maintain their bodyweight at $\sim 85-90 \%$ for the duration of the study. Training in the operant chambers commenced once the goal body weight had been reached.

\section{Operant training and testing.}

The training and testing sessions were carried out in standard operant conditioning chambers (Coulbourn Instruments, Allentown, PA) and operated using Graphic State Notation 3.0 software (www.coulbourn.com). Chambers were enclosed in ventilated and sound attenuating cubicles to prevent disruption during testing. Operant chambers were equipped with one medially located nose poke portal connected to a food hopper flanked by two retractable levers, one active and one inactive. Pressing on the active lever results in delivery of a manually programmed number of reinforcers. A dim house light, located on the wall above the nose-poke portal and retractable levers, was on for the duration of all sessions. Stimulus lights were present above each lever. A green light was illuminated for 3 seconds upon pressing of the active lever. Once rats had reached $\sim 85 \%$ of their original body weight, daily 30 -minute training sessions were initiated. During the first training session, a pellet was placed on the active lever to shape the rats behaviour and facilitate lever pressing. Training sessions continued until the rats 
obtained a stable level of responding (defined as two consecutive sessions in which $>100$ pellets were earned) on a fixed-ratio 1 (FR1) schedule of reinforcement, when one bar press resulted in the delivery of one $45 \mathrm{mg}$ pellet. Once criterion was achieved, rats were then trained on a fixed-ratio 2 (FR2) schedule of responding, during which 2 active lever presses were required to obtain one $45 \mathrm{mg}$ pellet. Once stable FR2 was achieved, training was continued under a fixed-ratio 4 (FR4) schedule of reinforcement. Finally, after FR4 responding was stable, rats were subjected to a progressive ratio (PR) schedule of reinforcement (Hodos, 1961), during which they were required to press the active lever an exponentially increasing number of times to receive one $45 \mathrm{mg}$ pellet. The series was derived from the equation [required active lever presses $=(5$ eellet number $\mathrm{x} 0.2)-5]$ (Richardson and Roberts, 1996). The first twenty pellets therefore require the following number of lever presses, respectively: $1,2,4,6,9,11,15,20,25,32,40,50,62,77,95$, $118,145,178,219,268$. After each reinforcer is delivered the number of presses resets, therefore, in order for a rat to obtain 20 pellets, it would need to press a total of 1378 times. Animals were tested on a PR schedule in 2-hour sessions for 7 consecutive days. Break point (BP) was defined as the last ratio that the rat successfully completed. This procedure was repeated (beginning at an FR4 schedule) for each of the 3 remaining dietary pellets offered over the course of the experiment. Rats responded rapidly enough with each new set of pellets on an FR4, that reverting to an FR1 schedule for each pellet type was unnecessary. Prior to the FR4 sessions for each pellet type, a bowl containing the pellets was left for $24 \mathrm{~h}$ in the home cage with the rats to prevent neophobia.

\section{Pellet reinforcers.}

All $45 \mathrm{mg}$ regular grain based, chocolate flavoured, sucrose, and high-fat chocolate flavoured pellet reinforcers used in the current study were obtained from 
Bioserv (Flemington, NJ), and were compatible with the Colbourne instruments delivery systems. Please see Table 1 for nutritional information pertaining to each type of pellet.

\section{Free choice test.}

On the day following the last PR session, rats were given access to $100 \mathrm{~g}$ of standard chow and $25 \mathrm{~g}$ of each of the four pellet types simultaneously in their home cage. The pellets were offered in glass dishes to which the rats had been previously exposed when pellets were offered prior to the FR4 training sessions. This test was used to determine which diet would be preferred when offered a simultaneous choice between the four pellet types.

\section{Statistical Analyses.}

To compare baseline body weight and food intake, as well as cumulative presses over all PR sessions, independent sample $t$-tests were performed. Total active bar presses during the final criterion FR-4 sessions were analyzed using a two-way ANOVA with pellet and genotype as independent variables $(4 \times 2)$. Repeated measures analysis of variance (ANOVA) was used to evaluate break point across testing days during PR testing. Two-way ANOVA (pellet by genotype) was used to assess whether sucrose pellets were consumed less by GHSR ${ }^{\mathrm{m} 1 / \mathrm{Mcwi}}$ rats. All statistical analyses were performed with SPSS 20.0.0 for Macintosh (IBM Corp., Armok, NY) and statistical significance was set at $\alpha=0.05$. All data are expressed as the mean $+/$ - standard error of the mean (SEM).

Results.

Body weight and standard chow intake.

Rats did not differ in body weight during the baseline period $\left(t_{(16)}=-.630, p=\right.$ $0.537)$ nor at the end of the experiment $\left(t_{(16)}=-1.368, p=0.190\right.$; see Figure 1A). As expected, the caloric restriction protocol resulted in all rats losing weight across the 
experiment $\left(F_{(1,16)}=98.404, p=0.000\right)$, however this was not influenced by genotype $\left(F_{(1,16)}=1.026, p=0.326\right)$. Time to reach $85 \%$ of bodyweight prior to operant conditioning did not differ between genotypes, with both reaching the stable goal weight by 23 days of caloric restriction (see Figure 1B), with body weight at $82.67(+/-0.23 \%)$ for the wildtype group and $84.17(+/-0.27 \%)$ for the GHSR ${ }^{\mathrm{m} 1 / \mathrm{Mcwi}}$ group prior to the onset of operant training. Standard chow intake at the end of the baseline period differed between groups, such that GHSR ${ }^{\mathrm{ml} / \mathrm{Mcwi}}$ rats consumed more chow than the WT rats $\left(_{(18)}\right.$ $=2.412, p=<.05$, see Figure 1C).

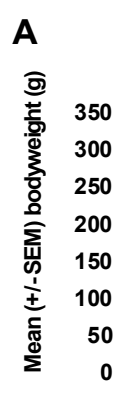

C

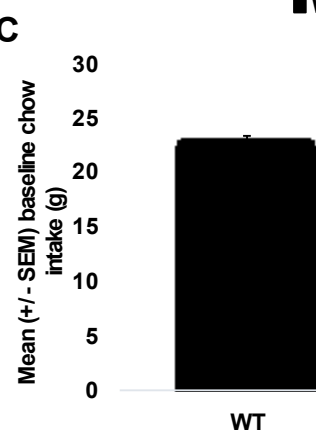

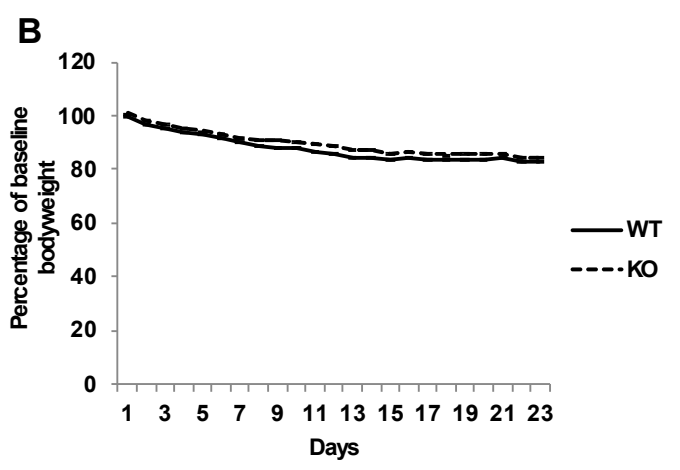

Figure 1. Bodyweight and chow intake. A) Bodyweight at baseline and at the end of the experiment. Body weights did not differ during baseline and while all animals lost a small amount of weight due to caloric restriction, no differences between GHSR ${ }^{\mathrm{m} 1 / \mathrm{Mcwi}}$ and WT rats were observed. B) Percentage of baseline body weight over caloric restriction. Time to reach $85 \%$ of bodyweight did not differ as a function of genotype. C) Baseline ad libitum chow intake was modestly higher in $\mathrm{GHSR}^{\mathrm{m} 1 / \mathrm{Mcwi}}$ rats, relative to WT rats. 
GHSR $^{m 1 / M c w i}$ and WT rats did not differ on Fixed ratio 4 responding (FR4) for regular, chocolate flavoured, sucrose or high-fat chocolate flavoured pellets.

All rats reached criterion quickly, pressing at least 100 times during the 30 minute test and, overall there were no differences between $\mathrm{GHSR}^{\mathrm{m} 1 / \mathrm{Mcwi}}$ and WT rats on number of active bar presses in the FR4 training session $\left(F_{(1,16)}=0.321, p=0.579\right.$; Figure 4). There was, however, a significant effect of pellet type $\left(F_{(3,48)}=5.406, p=0.003\right)$ on number of active lever presses. Pairwise comparisons revealed that rats pressed less for sucrose pellets as compared to regular pellets $(p=0.022)$, with no other differences observed between pellet types (all comparisons, $p>0.05$ ) and no interaction between pellet type and genotype $\left(F_{(3,48)}=0.199, p=0.896\right)$.

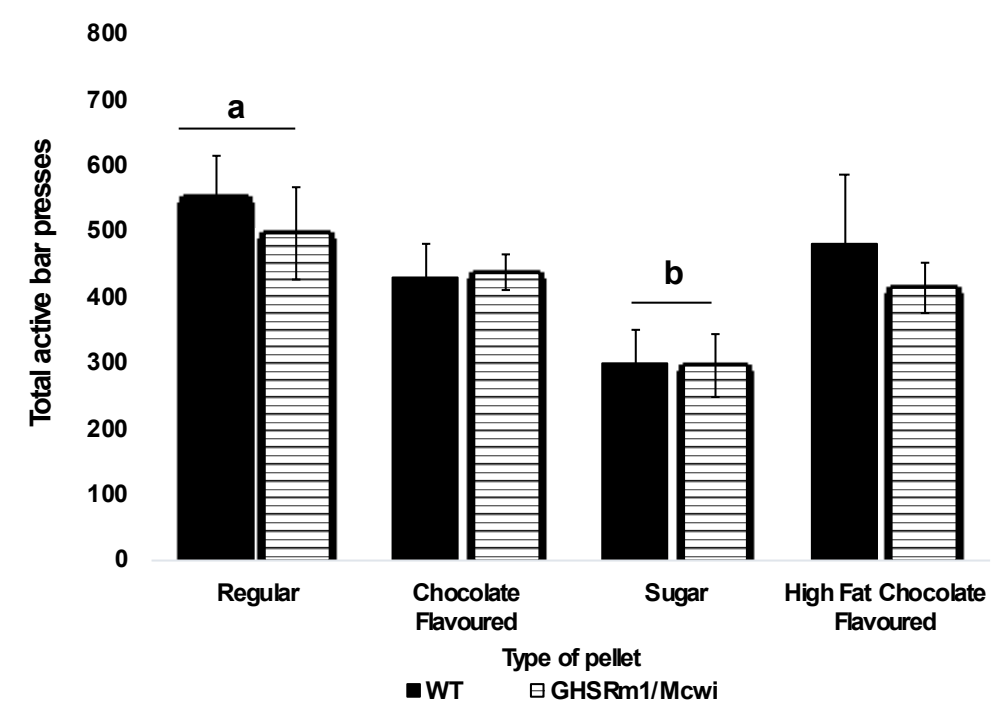

Figure 2. Fixed-ratio 4 responding. Total number of bar presses during the 30 -minute fixed ratio-4 (FR4) test on the second day of criterion. GHSR ${ }^{\mathrm{m} 1 / \mathrm{Mcwi}}$ and WT rats did not differ on the number of presses to obtain any of the different pellet types.

GHSRm1/Mcwi rats were less motivated to obtain sucrose pellets under a progressive ratio schedule of reinforcement.

Rats did not differ in how long it took them to reach criterion (on FR-4) to begin the initial PR tests $\left(t_{(16)}=-.516, p>0.5\right)$, with $\mathrm{GHSR}^{\mathrm{m1} / \mathrm{Mcwi}}$ rats taking an average of 
16.89 days (+/-1.7) and WT rats taking an average of 18.1 days $(+/-4.3)$ to reach stable responding on an FR4 schedule (see Figure 3A). The average number of active lever presses for all four types of pellets over all PR test days is shown in Figure 3B. Overall, WT rats pressed more frequently than GHSR ${ }^{\mathrm{m} 1 / \mathrm{Mcwi}}$ rats (main effect of genotype: $F_{(1,16)}=$ $5.558, p=0.031)$. There was also a significant effect of pellet type $\left(F_{(3,48)}=10.259, p=\right.$ 0.000), such that rats pressed the active lever less for high-fat chocolate flavored pellets, than for all other pellet types (pairwise post-hocs $p<0.05$ ) and pressed less for sucrose than for chocolate flavor pellets $(p<0.05)$. They pressed similarly for regular grain based and sucrose pellets $(p>0.05)$. The pellet by genotype interaction did not reach significance $\left(F_{(3,48)}=1.846, p=0.151\right)$, although as Figure 3B shows, $\mathrm{GHSR}^{\mathrm{m} 1 / \mathrm{Mcwi}}$ rats pressed fewer times for the sucrose pellets than WT rats, which contributed to the significant effect of pellet type.
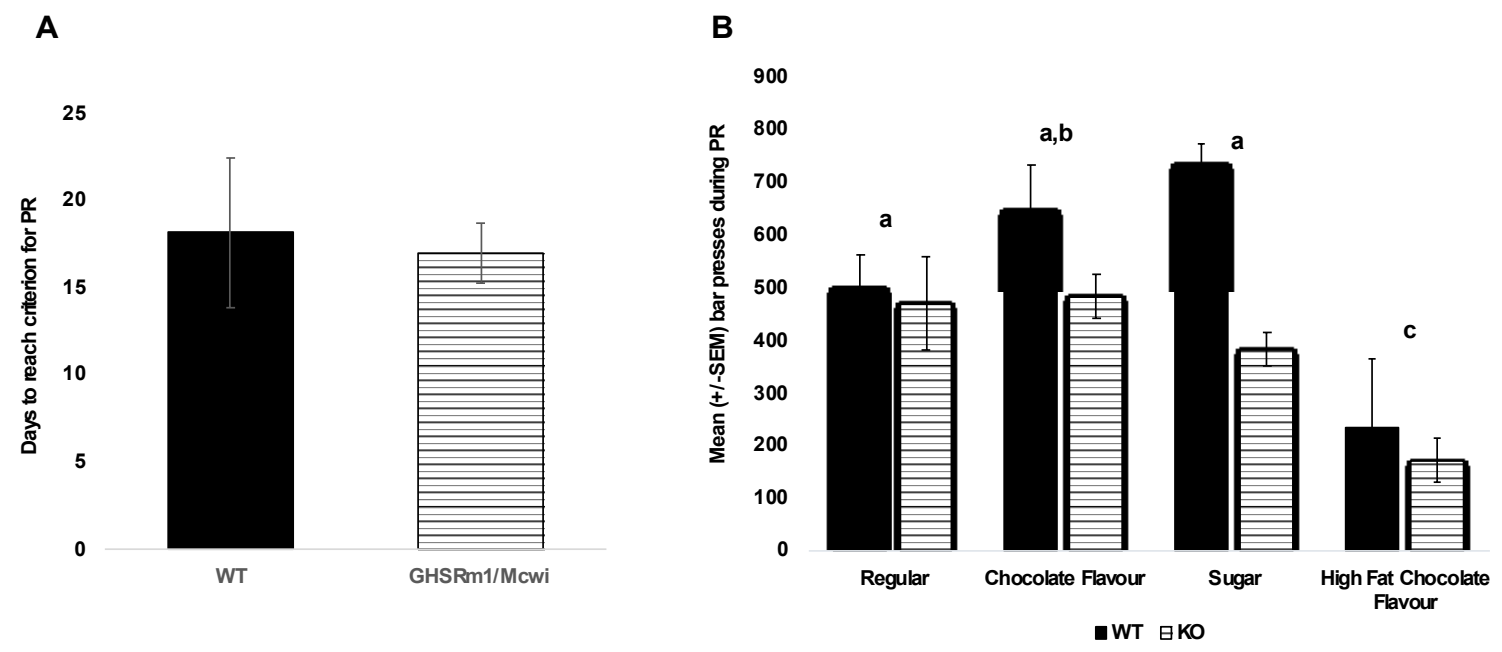

Figure 3. PR responding. A) Days to reach PR criterion. Time to reach criterion did not differ as a function of genotype. B) Bar presses over all PR sessions (7 days). WT rats pressed the active lever more often than GHSR ${ }^{\mathrm{m} 1 / \mathrm{Mcwi}}$ rats. All rats pressed the least for high-fat chocolate pellets (a,b, $\mathrm{p}<0.05 ; \mathrm{a}, \mathrm{c}, \mathrm{p}<0.05)$. 
Breakpoint over PR days.

During the 7 days of progressive ratio testing for regular grain-based pellets, there was no effect of time $\left(F_{(6,96)}=2.170, p>0.05\right)$, of genotype $\left(F_{(1,16)}=0.365, p>0.05\right)$, or a time by genotype interaction $\left(F_{(6,96)}=0.556, p>0.05\right)$ on breakpoint achieved, see Figure 4A. In contrast, when chocolate pellets served as the reinforcer, GHSR ${ }^{\mathrm{m} 1 / \mathrm{Mcw}}$ rats took 2 days to reach a similar level of responding to that of the WT rats, although behaviour was similar by day 3 of testing and onward (time*genotype interaction: $F_{(6,96)}$ $=4.783, p=0.000)$. Overall, however, there was no significant effect of genotype $\left(F_{(1,16)}\right.$ $=1.038, p>0.05)$, see Figure 4B. GHSR ${ }^{\mathrm{m} 1 / \mathrm{Mcwi}}$ rats had lower breakpoints than WT rats when pressing for sucrose pellets, resulting in a significant effect of genotype $\left(F_{(1,16)}=\right.$ 9.987, $p=0.006$, see Figure 4C). There was also an effect of time $\left(F_{(6,96)}=4.871, p=\right.$ $0.000)$, with rats pressing more at time 5 than at other times $(p<0.05)$, see Figure $4 \mathrm{C}$. There was no time by genotype interaction $\left(F_{(6,96)}=0.453, p=0.841\right)$. Although breakpoints for high-fat chocolate flavour pellets were lower on days 6 and 7 (significant main effect of time: $F_{(6,96)}=5.342, p=0.000$; pairwise comparisons for day 6 and $7, p<$ 0.05 , see Figure 4D), the genotype effect did not reach significance $\left(F_{(1,16)}=2.150, p=\right.$ $0.162)$.

Cumulative bar presses over PR sessions.

In addition to breakpoint, we measured the cumulative number of active lever presses rats performed over the 7-day PR testing period. GHSR ${ }^{\mathrm{m} 1 / \mathrm{Mcw}}$ and $\mathrm{WT}$ rats did not differ in number of presses for regular grain $\left(\mathrm{GHSR}^{\mathrm{m} 1 / \mathrm{Mcw}}: 3748+/-484\right.$ vs. WT: $3483+/-471 ; t_{(16)}=-3.92, p=0.700 ;$ Figure $\left.4 \mathrm{~A}\right)$, chocolate flavoured $\left(\mathrm{GHSR}^{\mathrm{m1} / \mathrm{Mcw}}: 3843\right.$ $+/-388$ vs. WT: $4529+/-877 ; t_{(16)}=.715, p=0.485$; Figure 4B), and high-fat chocolate flavoured pellets (GHSR ${ }^{\mathrm{m} 1 / \mathrm{Mcw}:} 103+/-192$ vs. WT: $1637+/-326 ; t_{(16)}=1.407, p=0.179$; 
Figure 4C). When tested with sucrose pellets, GHSR ${ }^{\mathrm{m} 1 / \mathrm{Mcwi}}$ rats pressed fewer times than $\mathrm{WT}_{\text {rats }}\left(\mathrm{GHSR}^{\mathrm{m1} / \mathrm{Mcw}}: 2747+/-290\right.$ vs. WT: $5097+/-982 ; t_{(16)}=2.292, p=0.036$;

Figure 4D). 

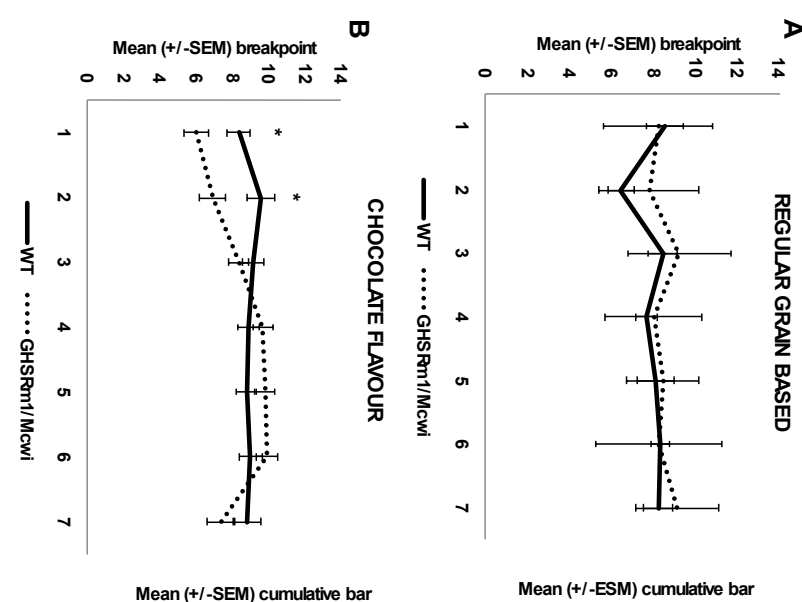

Mean $(+/$-SEM) cumulative bar
presses over all PR sessions

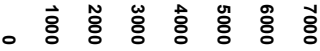

presses over
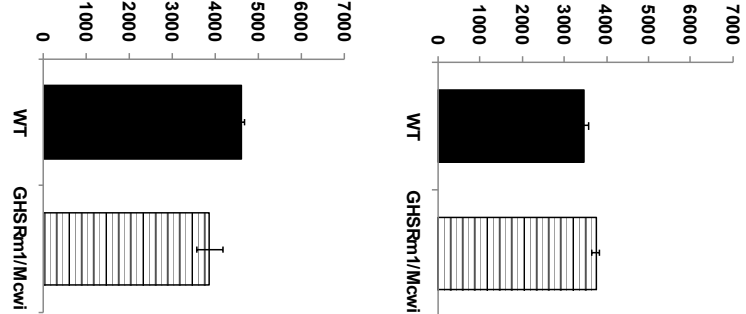

Meam (+/-SEM) breakpoint
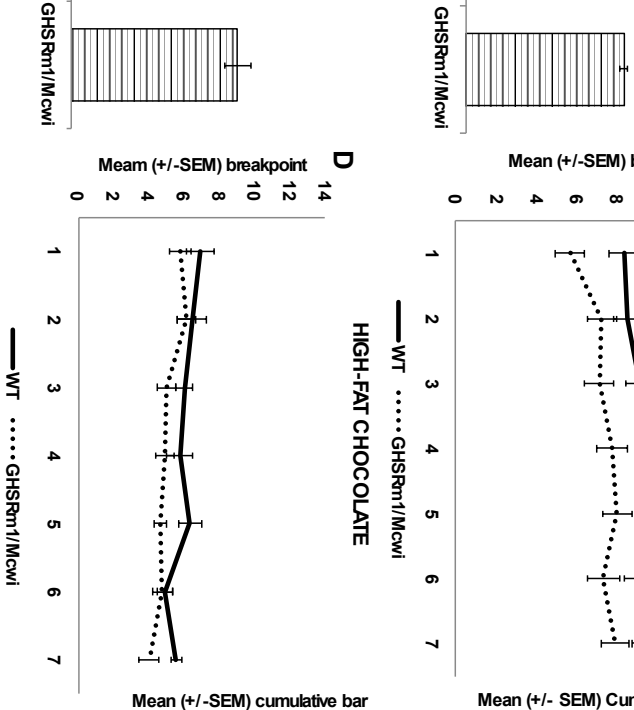

Mean (+/-SEM) breakpoint

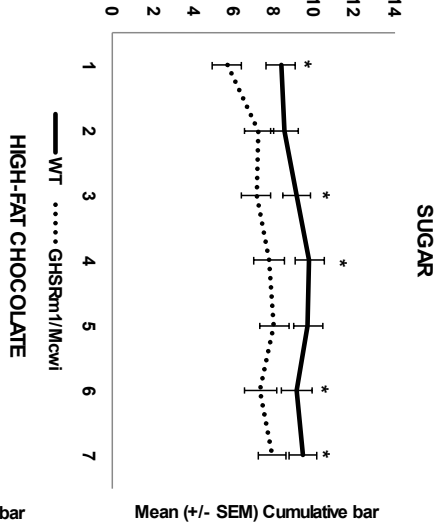

over all PR sessions

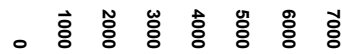

。
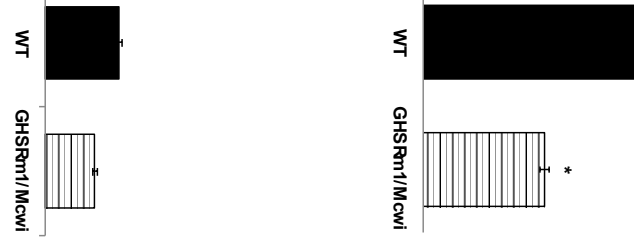

Figure 4. Breakpoint and cumulative bar presses. A) Breakpoint for the 2-hour PR session for regular purified pellets (left-hand panel) and cumulative bar presses over all 7 days of PR testing (right-hand panels); B) Chocolate-flavoured pellets; C) Sucrose pellets; D) High-fat chocolate-flavoured pellets. WT and GHSR ${ }^{\mathrm{m} 1 / \mathrm{Mcwi}}$ rats had comparable breakpoints for all types of pellets except for sucrose. GHSR ${ }^{\mathrm{m} 1 / \mathrm{Mcwi}}$ rats less effort compared to the WT rats $(* p<0.05)$ 
Free choice pellet preference test.

Intake of each type of pellet and lab chow was measured after 2 hours (see Figure

5). When presented with the 4 pellet types concurrently, the rats did not have a preference for a specific type of pellet over another $\left(F_{(3,48)}=2.245, p<0.05\right)$ and there was no pellet by genotype interaction $\left(F_{(3,48)}=1.357, p=0.267\right)$. However, there was a main effect of genotype, with GHSR ${ }^{\mathrm{m} 1 / \mathrm{Mcwi}}$ rats consuming fewer total pellets overall, as compared to WT rats $\left(F_{(1,16)}=6.033, p=0.026\right)$. There was no effect of genotype on amount of chow eaten during the free choice test (data not shown).

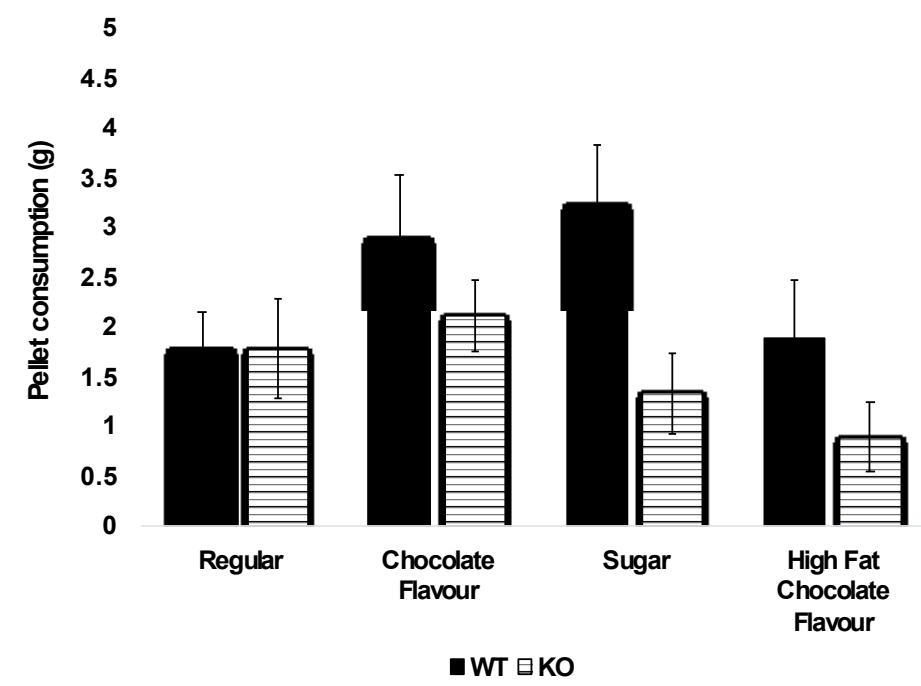

Figure 5. Free choice pellet test. Grams of pellets consumed during the two-hour free choice test at the end of operant testing. WT and GHSR ${ }^{\mathrm{m} 1 / \mathrm{Mcwi}}$ rats did not differ in the amount of regular, chocolate-flavoured or high-fat chocolate flavoured pellets they consumed during the two-hour period. Genotype did have an effect on pellet consumption, but this was irrespective of pellet type $\left({ }^{*} p<.05\right)$. 


\section{Experiment 1.2: GHSR $^{\mathrm{m} 1 / \mathrm{Mcwi}}$ rats spend less time in a sugar-paired compartment when conditioned in a hungry state, but when satiated during testing.}

\section{Materials and Methods.}

Animals.

Adult male wild-type (WT; $n=10)$ and $\mathrm{GHSR}^{\mathrm{m} 1 / \mathrm{Mcwi}}(n=12)$ fawn-hooded

hypertensive $(\mathrm{FHH})$ rats described for experiment 1.1 and derived from our in-house breeding colony were used in this experiment. General housing conditions were also as described above. Rats had ad libitum access to standard laboratory chow and water, except where stated otherwise.

\section{Apparatus.}

CPP conditioning and testing sessions were conducted in custom made Plexiglas CPP chambers (Plastics Ottawa, see Appendix B). To enable differentiation between the compartments based on contextual cues, one half of each box had white walls with a smooth white floor and the other half had black walls and floor with a wire mesh covering and were connected by a small gray centre compartment. During conditioning sessions, the middle section was closed via guillotine doors, confining rats to either the black or white compartment with the appropriate reinforcer. On test days, the doors were removed to allow rats free access to either compartment. The CPP chamber was cleaned with $70 \%$ ethanol solution and dried with paper towel after each session, to eliminate any odor cues left behind by previous rats.

\section{Conditioned Place Preference.}

Prior to conditioning, rats were provided with ad libitum access to the sucrose pellets used during conditioning for a $24 \mathrm{~h}$ period in their home cages, to prevent neophobia. On Day 1, a pre-conditioning test was conducted to determine initial side preference while no food was present in the CPP chamber. Rats were transported into the testing room and permitted to acclimate to the testing room for 20 minutes prior to 
testing. Upon the start of the pre-test, rats were placed into the middle compartment of the apparatus and doors were removed to allowed free access to either side of the chamber for 20 minutes. Initial preference for the black or white compartments was determined by which side they spent the most time in during the 20-minute exposure.

Conditioning sessions occurred from days 2 through 9, during which rats were confined for 20 minutes to either the dark chamber paired with 10 grams of regular chow, or the white chamber paired with 6 grams of sugar pellets. The placement of rats into either the light or dark side on the first conditioning day was counterbalanced and alternated each consecutive day for the next 7 conditioning days. After 8 conditioning days, rats were returned to their home cage. On the following day, a preference test was conducted. Rats had ad-libitum access to standard chow in their home cages throughout conditioning and prior to the preference test. No food was present in the chamber during the preference test, and animals had free access to both sides of the chamber for 20 minutes.

To investigate whether elevating endogenous ghrelin levels by testing rats under food restriction might exacerbate any effects of genotype, on days 11 through 17, rats were food restricted to 4 hours of ad-libitum access to standard chow in their home cages daily during the light phase (11:00h to $15: 00 \mathrm{~h})$. A second round of conditioning occurred on days 18 through 25 . Rats were conditioned in the morning hours (prior to their daily feeding at 11:00 h). After conditioning sessions, rats were returned to their home cages where they had access to food for the 4-hour window. On day 26, a second preference test was conducted, $18 \mathrm{~h}$ after the rats' last access to food. No food was available in the 
apparatus and rats had were free to move between the light and dark compartments for 20 minutes.

A third round of conditioning occured on days 27 through 34, however, the rats were conditioned in dim light as opposed to the standard lighting used in the previous conditioning and test sessions, in order to attenuate any potential anxiogenic effects of the testing conditions/white chamber. After a final conditioning session on day 34, rats were given ad-libitum access to regular chow in their home cages before the final preference test on Day 35. The final CPP test was therefore conducted in dim light and in presumably sated rats. As usual, no food was present during the CPP test and rats were free to move between both the light and dark compartments for $20 \mathrm{~min}$. Conditioning and test sessions were video recorded and later scored to determine place preference. All video scoring was conducted by an observer blind to group membership.

\section{C-fos immunohistochemistry.}

60 minutes following the final CPP test session, rats were given an overdose of Nembutal (intraperitoneally, i.p.) and transcardially perfused with $4 \%$ paraformaldehyde (PFA). Brains were then extracted and post-fixed in 4\% PFA for 24 hours, followed by cryo-protection in a $30 \%$ sucrose solution in $0.1 \mathrm{M}$ phosphate buffer (PB) solution and stored at $4^{\circ} \mathrm{C}$ until sectioning. To prepare brains for sectioning, brains were frozen with dry ice and sliced into $50-\mu \mathrm{m}$ coronal sections using a cryostat (LEICA CM1900, $-21^{\circ} \mathrm{C}$ ). Free floating sections were evenly distributed in a 6-series order and stored in $0.1 \mathrm{M} \mathrm{PB}$ prior to immunohistochemistry (IHC) procedures.

Coronal slices through the VTA $(-5.20 \rightarrow-6.30$ Bregma), NAcc core and shell $(2.70 \rightarrow 1.70$ Bregma $)$, and MePV $(-2.56 \rightarrow-3.30$ Bregma) were used for immunohistochemistry using a rabbit anti-C-fos antibody (Oncogene Science, Boston, 
MA, USA). Free-floating sections were washed in $0.1 \mathrm{M} \mathrm{PB}, \mathrm{pH} 7.4$, and incubated at room temperature (RT) for 30 min in a quenching solution made of $\mathrm{PB}$ and $1 \% \mathrm{v} / \mathrm{v} \mathrm{H}_{2} \mathrm{O}_{2}$. Sections were then rinsed in cold PB and incubated for one hour at RT in a pre-blocking solution ( $0.3 \%$ Triton $\mathrm{X}-100$ in PB and bovine serum albumen (BSA)). The sections were then incubated in the primary antibody solution, with an affinity-purified rabbit polyclonal antibody raised against C-fos [1:20,000] combined with $0.3 \%$ Triton X-PB and BSA. Sections were incubated with the primary antibody for $48 \mathrm{~h}$ at $4{ }^{\circ} \mathrm{C}$, washed with cold PB and incubated for one hour at RT with the secondary antibody, biotinylated anti-rabbit IgG raised in donkey ([1:250]; Jackson ImmunoResearch Laboratories, Inc., Westgrove, PA, USA). Sections were washed again in cold PB and incubated for one hour at RT with an avidin-biotin-peroxidase complex (Vectastain Elite ABC Kit; Vector Laboratories Inc. Burlington, ON, Canada). 3,3'-diaminobenzidine (DAB; 0.05\%) in PB was then added to each well. Sections were then incubated on an orbital shaker for $5 \mathrm{~min}$, and then $0.01 \% \mathrm{H}_{2} \mathrm{O}_{2}$ was added to the sections for an additional 5 minutes. After this final colorometric reaction, sections were washed with cold PB and mounted onto gelcoated slides. Slides were then dehydrated through a series of ascending alcohol concentrations, submerged in Clearene (Surgipath, Leica Microsystems Inc., Concord, ON, Canada), and cover-slipped with Permount (Fisher Scientific, Toronto, ON, Canada). Image analysis.

Brain tissue sections were imaged using an Olympus BX51 microscope and an MBF bioscience camera (Model: 2000C-00-GE-MBF) connected to a desktop computer with Windows XP operating system (Microsoft Corp., Redmond, WA, USA). Cells immunopositive for C-Fos were counted manually from the captured images using ImageJ64 (NIH freeware). The mean number of $\mathrm{C}$-fos positive cells per region was 
calculated for each rat by counting two coronal sections within the VTA, MePV, and the NAcc showing the highest density of labeled cells, and the average of the two sections was taken as the count for that region.

Statistical analyses.

Absolute time spent in the sugar-paired chamber was the dependent variable of interest for determining differences between genotypes on CPP test days. Differences between GHSR ${ }^{\mathrm{m} 1 / \mathrm{Mcwi}}$ and WT rats were analyzed using t-tests. Differences in C-fos counts between GHSR ${ }^{\mathrm{m} 1 / \mathrm{Mcwi}}$ and WT rats for each brain region were also assessed using Students t-tests. The alpha level for all comparisons was set at $p<0.05$ for all analyses, and all data reported are mean $+/-$ SEM.

Results.

GHSRm1/Mcwi rats spend significantly less time in environments previously paired with sucrose, when tested under sated and dim light conditions.

Following 8 conditioning sessions while rats received ad-libitum access to standard chow in their home cage, there were no differences between GHSR ${ }^{\mathrm{m} 1 / \mathrm{Mcwi}}$ and WT rats in absolute time spent in the sugar-paired chamber on Test Day 1 (TD1) $\left(t_{(20)}=\right.$ 0.755, $p>0.05$; see Figure 1). On Test Day 2 (TD2) when rats were tested while food restricted, GHSR ${ }^{\mathrm{m} 1 / \mathrm{Mcwi}}$ and WT rats again exhibited no difference in absolute time spent in the previously sugar-paired chamber $\left(t_{(20)}=0.927, p>0.05\right.$; see Figure 1), although both groups did spend more time in the sucrose-paired chamber on this test than during pre-conditioning. After a further 7 conditioning trials carried out in dim light under foodrestricted conditions, rats were given ad-libitum access to regular chow in their home cage, before a third and final preference test on the following day. This test was thought to better reflect hedonic feeding, suggesting that any learned association between the context and sucrose was still potent, even in the absence of hunger at the time of testing. 
During this test, GHSR ${ }^{\mathrm{m} 1 / \mathrm{Mcwi}}$ rats spent significantly less time in the white chamber than $\mathrm{WT} \operatorname{rats}\left(t_{(20)}=2.118, p<0.05\right)$.

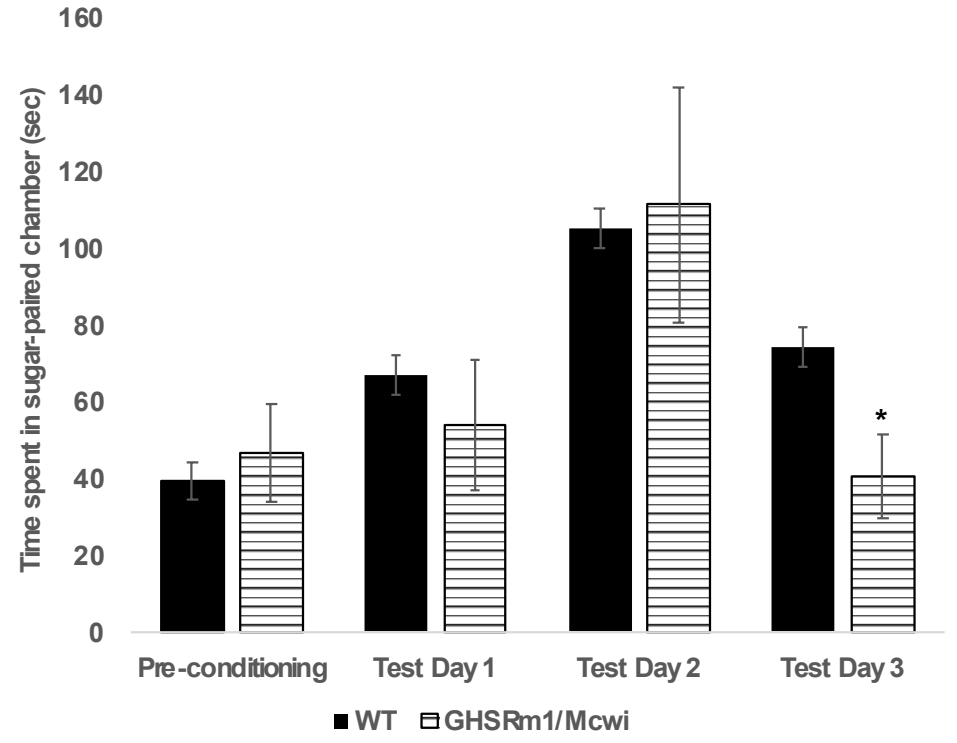

Figure 1. Absolute time spent in the sugar-paired chamber during pre-conditioning and test days. GHSR ${ }^{\mathrm{m} 1 / \mathrm{Mcwi}}$ and $\mathrm{WT}$ rats showed no differences in absolute time spent in the white chamber on Test Day $1\left(t_{(20)}=0.755, p>0.05\right)$ or Test Day $2\left(t_{(20)}=\right.$ $0.927, p>0.05)$. GHSR ${ }^{\mathrm{m} 1 / \text { Mcwi }}$ rats spent significantly less time in the white chamber than WT rats on Test Day $3(* p<0.05)$.

GHSR $^{\text {mI/Mcwi }}$ rats display attenuated cFos expression in the ventral tegmental area. $\mathrm{GHSR}^{\mathrm{m} 1 / \mathrm{Mcwi}}$ rats displayed significantly fewer cFos immunoreactive nuclei in the

VTA compared to WT rats following the third CPP test $\left(t_{(20)}=2.338, p<0.05\right)$, see

Figure 2. When calculated as a percentage, the number of cFos IR cells in the VTA of $\mathrm{GHSR}^{\mathrm{m} 1 / \mathrm{Mcwi}}$ rats was approximately $41 \%$ of that seen in WT rats. 
A

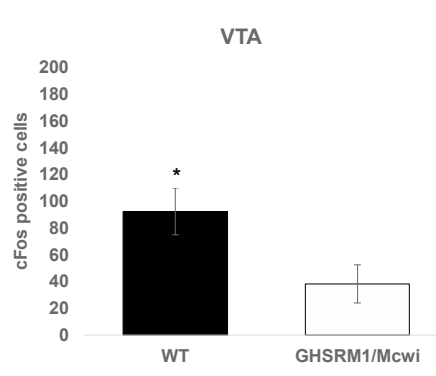

C

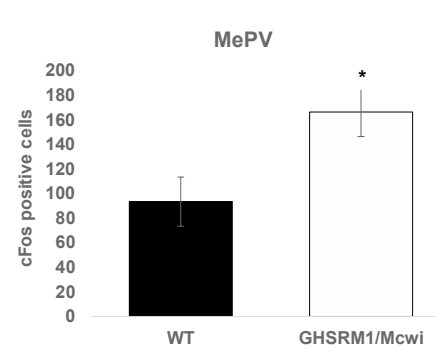

B

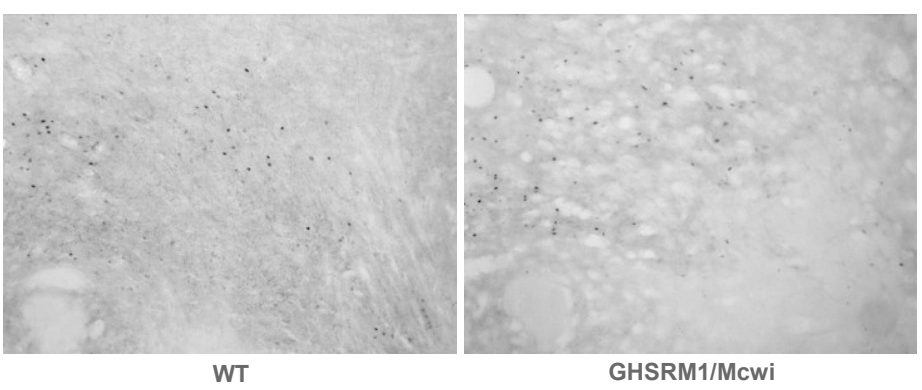

D

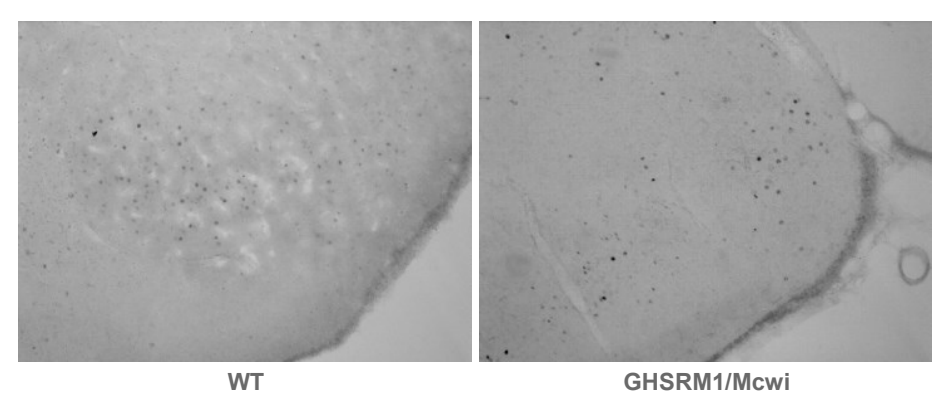

Figure 2. Neuronal activation following CPP. A) Bilateral quantification of cFOS positive nuclei in the VTA and B) Unilateral representative coronal photomicrographs of WT and GHSR ${ }^{\mathrm{m} 1 / \mathrm{Mcwi}} \mathrm{C}$-fos expression in the VTA (Bregma $-5.80 \mathrm{~mm} ; 10 \mathrm{X}$ ). GHSR $^{\mathrm{m} 1 / \text { Mcwi }}$ rats displayed significantly less C-fos immunoreactivity in the VTA $\left({ }^{*} p<0.05\right)$. C) Bilateral quantification of C-fos positive nuclei in the MePV and D) Unilateral representative coronal photomicrographs of WT and GHSR ${ }^{\mathrm{m} 1 / \mathrm{Mcwi}}$ rat C-fos expression in the MePV (Bregma $-2.80 \mathrm{~mm}$; 10X magnification). GHSR ${ }^{\mathrm{Mc} / \mathrm{Mwi}}$ rats display greater $\mathrm{C}$-fos immunoreactivity in the MePV compared to WT rats $(* p<0.05)$.

GHSR $^{\text {m1/Mcwi }}$ animals display augmented $C$-fos expression in the posteroventral medial amygdala $(\mathrm{MePV})$.

GHSR $^{\mathrm{m} 1 / \mathrm{Mcwi}}$ rats displayed significantly more C-fos immunoreactive nuclei in the MePV compared to WT rats following test day $3\left(t_{(20)}=6.393, p<0.05\right)$, see Figure $3 \mathrm{~A}$. When calculated as a percentage, the number of C-fos IR cells in the MePV in WT rats was approximately $56 \%$ of that seen in $\mathrm{GHSR}^{\mathrm{m} 1 / \mathrm{Mcwi}}$ rats.

GHSR $^{m 1 / M c w i}$ and WT rats exhibited similar levels of C-fos expression in the NAcc core and shell regions.

There were no significant differences in the number of $\mathrm{C}$-fos immunoreactive nuclei between GHSR ${ }^{\mathrm{m} 1 / \mathrm{Mcwi}}$ rats and WT rats in the NAcc, either in the core $\left(t_{(20)}=\right.$ $0.082, p>0.05)$ or shell regions $(t(20)=0.007, p>0.05)$ regions (data not shown). 


\section{Experiment 1.3.1: Demonstrating localization of AAV infusion using green- fluorescent protein and tyrosine hydroxylase immunohistochemistry.}

Materials and Methods.

Transgenic mice.

The transgenic mice used for GHSR rescue experiments were originally obtained from the University of Texas Southwestern Medical Centre (Zigman et al., 2005), and an in-house colony was maintained in our facility. Genotype was determined using standard polymerase chain reaction (PCR) performed on ear tissue punches obtained at the time of weaning (Transnetyx). Originally, GHSR-null mice were created by inserting a loxPflanked transcriptional blocking cassette (TBC) into a putative intron situated downstream of the transcriptional start site and upstream of the translational start site of the murine GHSR gene in C57BL6/J mice (see Appendix A). This approach to gene knockout, in which the GHSR locus was modified by the addition of a loxP-flanked TBC, rather than by the removal of a critical exon or exons, was generated to allow for Cre recombinase (CRE) driven reinstatement of GHSR in future experiments (Zigman et al., 2005). GHSR-null mice ( 2 copies of the recombinant GHSR allele) and their wild type (WT) littermates ( 2 copies of the wild-type GHSR allele) are viable, and were used for this study.

Adult male GHSR null mice $(n=20)$ and their WT littermates $(n=20)$ from our inhouse colony were utilized in this study. Mice were housed individually at the start of the experiment in a temperature and humidity-controlled vivarium with a 12:12 light/dark schedule (Lights on: 07:00 to 19:00). All mice had ad libitum access to water and nutritionally complete standard laboratory chow (Teklad Global 14\% Protein Rodent Maintenance Diet 2014, Harlan Laboratories, Madison, WI, USA; percent of kilocalories 
derived from fat: 13 , protein: 20 , carbohydrates: $67 ; 2.9 \mathrm{kcal} / \mathrm{g}$ ) for the duration of the study.

\section{Adeno-associated viral vectors.}

Delivering CRE via adeno-associated viral vectors (AAVs) to GHSR null mice

allows for reinstatement of the GHSR in adult mice with spatiotemporal precision, enabling the exploration of the role of specific regions of interest on particular behaviours. We tested GHSR null mice for sucrose preference using a two-bottle choice test, to investigate potential genotypic disparities in sucrose preference. Subsequently, we introduced CRE fused to a green-fluorescent protein (GFP) reporter gene via AAV into the VTA to determine the ability of the gene rescue to restore this behaviour to the level of WT mice. Two AAVs were used in this study: AAV9.hSyn.eGFP.WPRE.bGH (Control, reporter-only virus; 8.88e13 GC/ml, "titer"; Yield (GC): 8.88e12; Lot CS0354) and AAV9.hSyn.HI.eGFP-Cre.WPRE.SV40 (“Active” Cre-virus; 5.54e13 GC/ml, "titer”; Yield (GC): 5.54e12; Penn Vector Core, Gene Therapy Program, UPENN). We divided GHSR null and WT mice into 4 groups to visualize which dose most efficiently transduces our region of interest. Three dilutions of the active virus $(1: 10 ; 1: 100$, and 1:1000) were tested as was one dilution of the inactive control virus (1:62.5).

Surgical delivery of adeno-associated viral vectors.

Surgery was conducted using a gaseous anaesthetic (Isofluorane/Oxygen mixture; 2-5\% delivered in $1 \mathrm{l} / \mathrm{min} \mathrm{O}_{2}$ ). Mice were anaesthetized and placed into a stereotaxic apparatus to allow for accurate localization of the target region (VTA). An incision was made in the skin above the skull, the skin was retracted and bregma was located. Following demarcation of the target infusion site (co-ordinates: AP: -2.95; ML: +/- 0.7; DV: -4.55), a small hole was drilled for insertion of the injector and delivery of the virus 
(bilateral infusions: rate: $75 \mathrm{nl} / \mathrm{min} / \mathrm{side}$; volume: $0.5 \mu \mathrm{l} / \mathrm{side}$ ). Infusions were delivered using a 33-gauge injector connected via PE 20 tubing to a $10 \mu 1$ Hamilton airtight syringe attached to a GenieTouch ${ }^{\mathrm{TM}}$ dual-syringe pump (Kent Scientific Corporation). The skin was then sutured and the incision site was disinfected, followed by topical analgesic application. Mice also received an injection of analgesic (Metacam) just prior to the beginning of surgery and for 3 days following to ensure sufficient pain relief. Mice lived for 3 weeks to determine whether viral transduction persists to this time point.

\section{Perfusion and Histology.}

Mice were injected with a lethal dose of Dorminal (1mg/kg i.p.; CDMV, Quebec, Canada) and perfused transcardially with $0.9 \%$ saline, followed by $4 \%$ paraformaldehyde. Brains were then extracted and post-fixed in 4\% paraformaldehyde for $24 \mathrm{~h}$, followed by submersion in a $30 \%$ sucrose solution (w/v) prior to sectioning. 40 $\mu \mathrm{m}$ sections containing the VTA (Figs. 55-63, Paxinos and Franklin, 2001) were sliced at $-21^{\circ} \mathrm{C}$ on a Thermoscientific ${ }^{\mathrm{TM}}$ Cryostat. Sections were placed into wells containing 0.1 M phosphate buffer and every sixth section was processed for GFP-immunofluorescence (GFP-IF) and tyrosine hydroxylase immunofluoresence (TH-IF).

\section{Immunohistochemical verification of viral transduction.}

To localize the viral transduction, we performed immunohistochemistry on a series of brain slices from the AAV infused mice. Standard immunofluorescence procedures were used to determine the site of viral infection. Briefly, free-floating sections were washed in $0.1 \mathrm{M}$ phosphate buffer $(\mathrm{PB}, \mathrm{pH}$ 7.4) followed by incubation at RT for a minimum of 30 minutes in a blocking solution comprised of $0.3 \%$ Triton-X, bovine serum albumin (BSA), and normal goat serum (NGS) in PB. Blocking solution was then removed and the sections were incubated for $24 \mathrm{~h}$ at room temperature (RT) in a 
chicken anti-GFP primary antibody (Abcam; 1:10,000 concentration; Product \#13970) solution containing Triton-X and BSA in PB. Sections were washed using $0.1 \mathrm{M}$ PB and incubated with an AlexaFluor ${ }^{\circledR} 488$ goat anti-chicken IgG secondary antibody (Jackson Immunoresearch Laboratories Inc., Westgrove, PA, USA; 1:250) for 1h at RT while protected from light. For sections that were also probed for $\mathrm{TH}$, sections were incubated with both the anti-GFP primary (Abcam) as well as a primary mouse anti-TH antibody (Immunostar; 1:20,000 concentration), to visualize whether virally transduced neurons also express TH, as our hypothesis suggests that GHSR activity on TH neurons likely play a role in enhancing the incentive salience for and/or reward derived from palatable foods. Upon completion of fluorescent staining, slices were mounted onto glass slides and allowed to dry in the dark. Slides were then cover-slipped using Fluoromount ${ }^{\circledR}$ mounting media containing 4',6-diamidino-2-phenylindole (DAPI) as a nuclear counterstain (Abcam). The outer perimeter of the slides were sealed with clear nail polish, and stored at $-20^{\circ} \mathrm{C}$ until imaging.

Sections from mice in Study 1.3.1 were imaged at 10x, 20x, and 40x magnification using an Olympus BX61 microscope. Images of the VTA were captured using InVitro software (Media Cybernetics). 

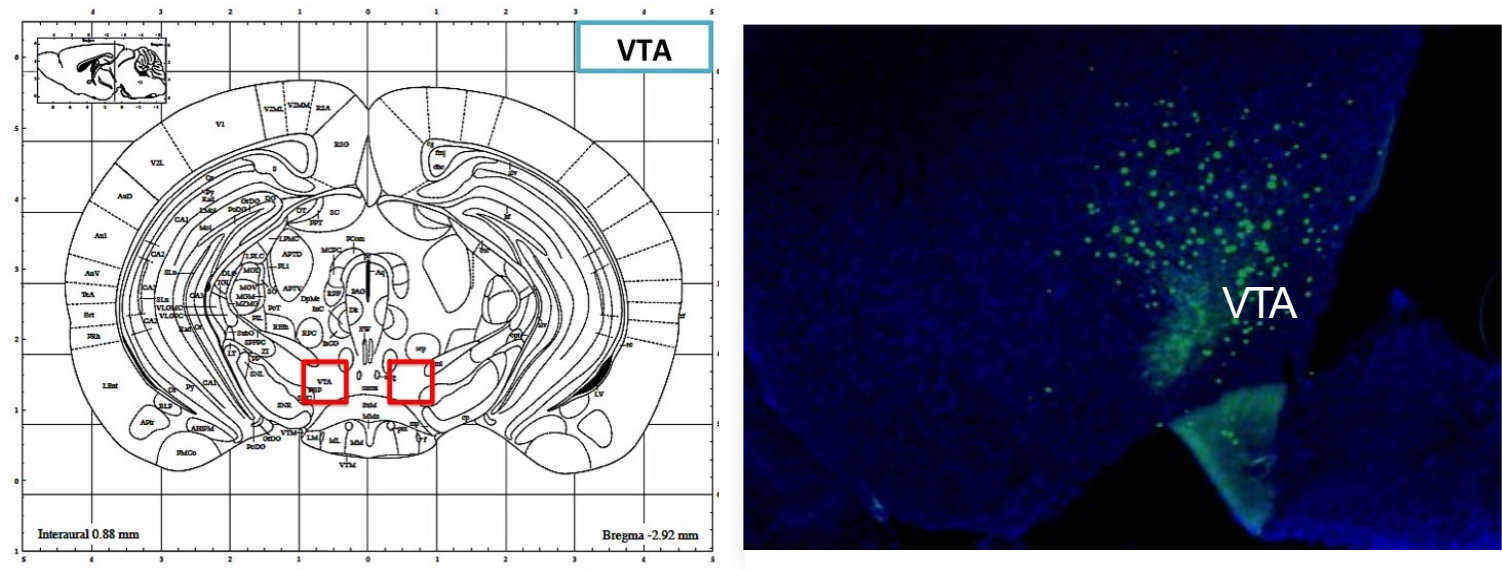

Figure 1. Immunohistochemical detection of GFP following AAV infusion, 3 weeks following infusion into the VTA. Image depicts a 1:10 dilution of the active virus. This served as the chosen concentration for the following studies, as it produced the greatest density of staining in the VTA, as visualized upon imaging. 

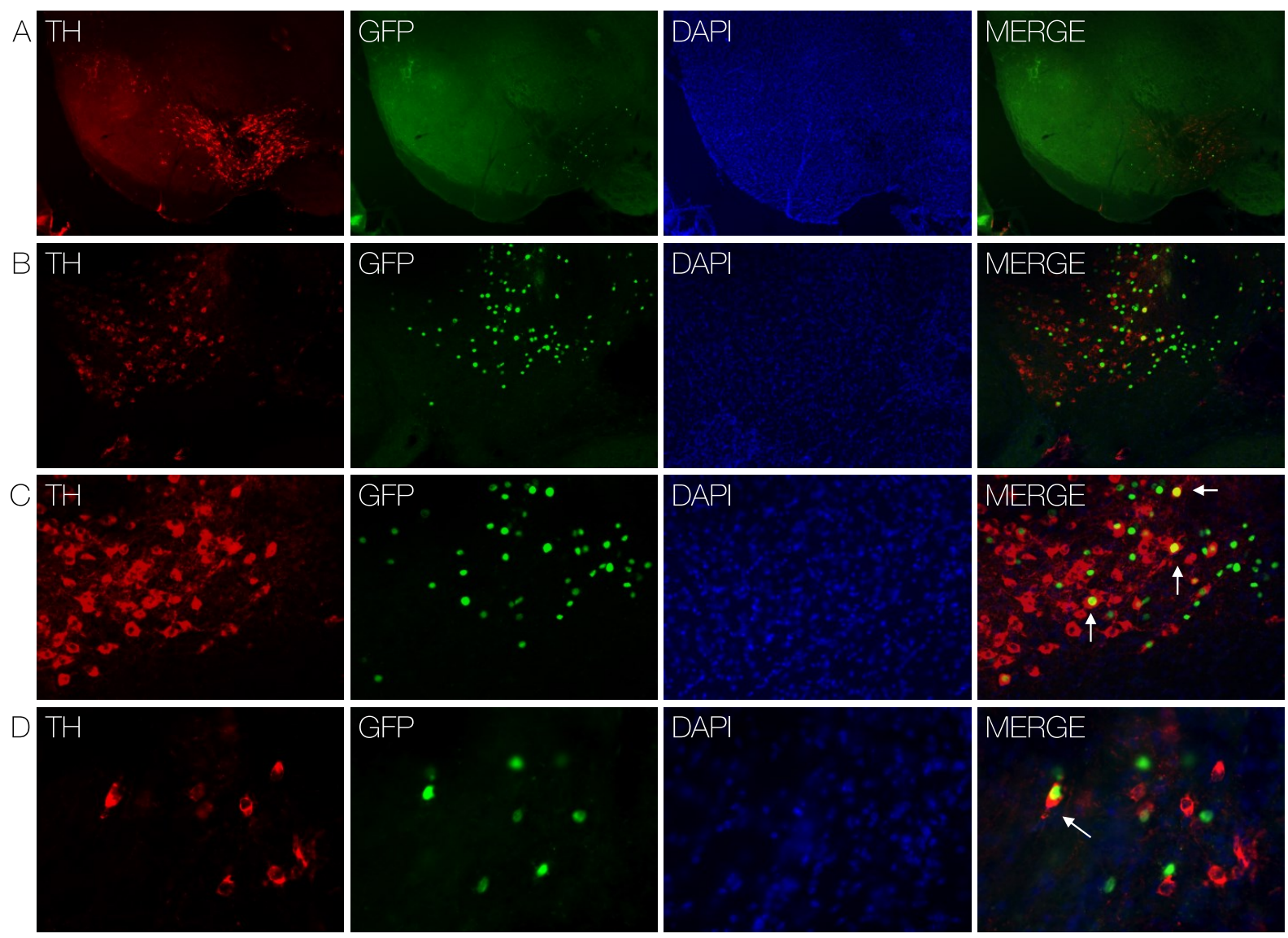

Figure 2. Photomicrograph depicting viral infusion into VTA, processed with DAPI, GFP and TH (1:10 dilution of AAV). A) Photomicrograph of the left VTA transduced with AAV at 4X; B) 10X; C) 20X; D) 40X. White arrows depict colocalization of GFP with TH.

Experiment 1.3.2: Rescue of the GHSR in the VTA enhances preference for sucrose solutions in a two-bottle choice test.

Materials and Methods.

Animals.

Adult male GHSR null $(n=14)$ and WT mice $(n=14)$ were used in this

experiment. Mice were housed individually at the start of the experiment in a temperature and humidity-controlled vivarium with a 12:12 light/dark schedule (Lights on: 07:00 to 19:00). All mice had ad libitum access to water and nutritionally complete standard laboratory chow (Teklad Global 14\% Protein Rodent Maintenance Diet 2014, Harlan 
Laboratories, Madison, WI, USA; percent of kilocalories derived from fat: 13, protein: 20, carbohydrates: $67 ; 2.9 \mathrm{kcal} / \mathrm{g}$ ) for the duration of the study.

Sucrose Preference Tests.

Following the baseline period, GHSR null and WT mice were given access to a $3.5 \%$ sucrose solution for $48 \mathrm{~h}$ in their home cage, in parallel to regular water access. Both solutions were offered in $100 \mathrm{ml}$ sipper tubes on top of the home cage. This concentration of sucrose was chosen as it has previously been shown to induce a preference in mice (Domingos et al., 2011). Sucrose intake was measured at $24 \mathrm{~h}$ intervals during the first 48 $\mathrm{h}$ of exposure. At the end of the initial $48 \mathrm{~h}$ exposure, sucrose was removed for 5 days. On the $6^{\text {th }}$ day, sucrose was re-introduced for $24 \mathrm{~h}$, and the solutions were weighed at $1 \mathrm{~h}, 2 \mathrm{~h}$, $4 \mathrm{~h}$, and $24 \mathrm{~h}$. Following a day with no access to sucrose, mice were again subjected to a $1 \mathrm{~h}$ sucrose preference test and intake was measured at 10,20,30 and 60 minutes.

Relative left-right positions of sucrose and water tubes during initial exposure and during tests was counterbalanced within each group and across testing days. Following sucrose testing, mice were subjected to stereotaxic surgery, during which either AAV-Cre (1:10) or AAV-eGFP (1:62) was infused into the VTA (see 1.3.1). Mice were allowed 3 weeks to recover in their home cage. At the end of the 3-week period, the mice were administered sucrose preference tests as in the pre-surgery phase of the experiment (a $24 \mathrm{~h}$ test, followed by a $1 \mathrm{~h}$ test). Percentage sucrose preference was calculated in all instances as: $[($ sucrose consumption $) /($ sucrose consumption + water consumption $)] * 100$. Baseline sucrose preference for each of the pre-surgery tests were subtracted from post-surgery tests to determine changes in preference. Mice were sacrificed by rapid decapitation the day following the last sucrose test. 
Surgical delivery of adeno-associated viral vectors.

Surgical procedures regarding delivery of the virus into the VTA were identical as

described for Experiment 1.3.1.

Verification of GHSR mRNA expression in the VTA.

$R N A$ extraction and $C D N A$ synthesis.

Upon decapitation, mouse brains were extracted and flash frozen in ice cold

ethanol and stored at $-80^{\circ}$ until RNA extraction. Brains were sectioned into $1 \mathrm{~mm}$ coronal sections at the level of the VTA using a stainless-steel mouse brain matrix and mouse brain atlas. Total RNA from bilateral hippocampal and VTA brain punches, obtained with a $15 \mathrm{~g}$ blunt needle, was isolated from homogenized brain punches using TRIzol (Life Technologies) and precipitated using linear acrylamide. The purity and concentration of extracted RNA was quantified by the ratio of absorbance at $260 \mathrm{~nm}$ over 280nm with a ThermoScientific Nanodrop 100 spectrophotometer (ThermoScientific, Rockford, Illinois). RNA quality was determined by gel electrophoresis. cDNA synthesis was conducted using the iScript cDNA synthesis kit (BioRad Laboratories Inc., Hercules, California). cDNA was stored in $-20^{\circ} \mathrm{C}$ until PCR was conducted.

Real-time quantitative polymerase chain reaction (RT-qPCR).

RT q-PCR was conducted on all cDNA samples to determine relative expression of target genes [ $\beta$-actin (reference gene) and GHSR (gene of interest)], using the $\Delta \Delta \mathrm{Ct}$ method. The WT GFP group served as the control group for analysis of relative gene expression, and the analysis for either regions was compared to this groups' corresponding expression in that region. $5 \mu 1$ of cDNA sample was added to each well of a 96-well PCR plate, followed by $2 \mu 1$ of working concentration of primer solution, $3 \mu 1$ of DEPC water, and $10 \mu 1$ of SsoAdvanced ${ }^{\mathrm{TM}}$ SYBR ${ }^{\circledR}$ Green Super Mix with Fluorescein (Bio-Rad Laboratories Inc, Hercules, California). Samples were run in duplicate 
alongside non-template controls (NTCs) and positive controls. The plates were run on a CFX Real-time PCR detection system (Bio-Rad) and data was analyzed using CFX Manager 3.0 software. The RT-q-PCR protocol consisted of 30 seconds at $95^{\circ} \mathrm{C}$, followed by 45 cycles of the following: 10 seconds at $95^{\circ} \mathrm{C}$ denaturing, 30 seconds at $55^{\circ} \mathrm{C}$ for annealing, and 20 seconds at $72^{\circ} \mathrm{C}$ for extension. The final steps include 1 minute at $95^{\circ} \mathrm{C}$ and 1 minute at $55^{\circ} \mathrm{C}$. Primers were initially tested for amplification efficiency using the standard curve method and yielded over 90\% efficiency. Primers were purchased from Eurofins Genomics (Huntsville, Alabama). See Table 2 for primer sequences used in this study.

Statistical Analyses.

24h sucrose preference data was analyzed using a two-way ANOVA (genotype $\mathrm{x}$ virus). All data from this experiment are expressed as mean \pm standard error of the mean (SEM). All statistical analyses were performed with SPSS 20.0.0 for Macintosh (IBM Corp., Armok, NY) and statistical significance was set at $\alpha=0.05$.

Results.

Verification of GHSR $m R N A$ in the VTA.

One- way ANOVA (genotype by virus) performed on relative gene expression values demonstrated that in the VTA there was a main effect of group $\left(F_{(3,19)}=4.174, p<\right.$ 0.05). The GFP GHSR null mice had virtually no GHSR in the VTA, as compared to all other groups $(p<0.023)$. The GHSR null CRE-GFP mice had comparable expression as seen in WT mice, both GFP only or WT CRE-GFP mice (post-hoc tests all $p>0.05$ ), indicating successful reinstatement of GHSR in this region. In the control region, the CA3 region of the hippocampus, relative expression also varied as a function of genotype $\left(F_{(3,15)}=11.868, p=0.001\right)$. WT mice were the only mice to express GHSR in this region (post-hoc comparisons between WT and null mice all $p<0.05$ ), which confirms that the 
GHSR null mice lack GHSR, and allows us to demonstrate a lack of viral transduction in the hippocampal region in these mice (see Figure 1).
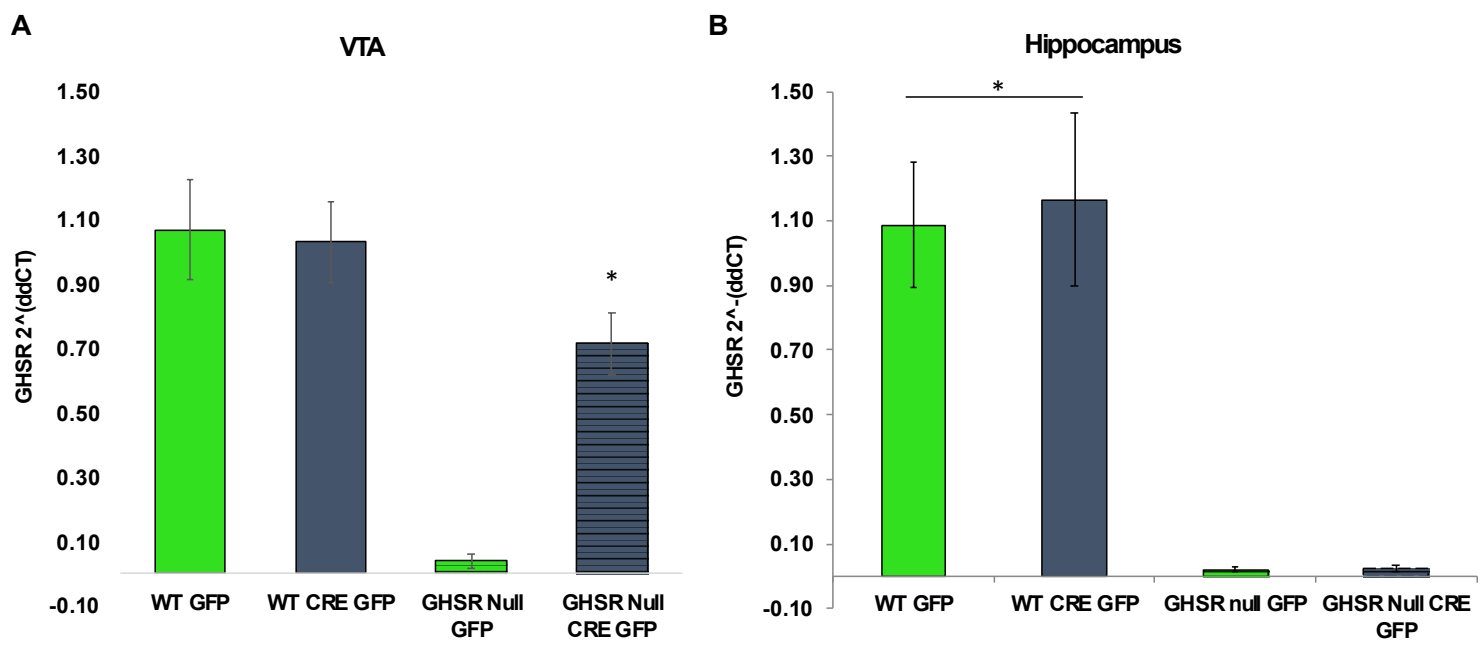

Figure 1. Relative GHSR expression. A) Relative expression in the VTA, where GHSR null mice delivered the GFP reporter virus were the only mice without GHSR expression $(*, p<0.05)$. B) Relative expression in the CA3 region of the hippocampus, where only WT show any GHSR expression, as expected (*, $p<0.05)$.

$24 h$ sucrose preference.

Prior to surgical delivery of the AAV, mice were tested for preference for a $3.5 \%$ sucrose solution over water across a $24 \mathrm{~h}$ period. Analysis of the preference scores of the GHSR null and WT demonstrated that GHSR null mice showed less of a preference for sucrose than WT mice $\left(F_{(1,23)}=4.231, p<0.05\right)$. Two-way ANOVA (genotype $\mathrm{x}$ virus) examining the change in sucrose preference from pre-surgery to post-surgery across all experimental groups found that there was a difference between groups $\left(F_{(3,23)}=3.577, p\right.$ $<0.05)$. Pairwise comparisons revealed that only the GHSR null mice that received the active CRE-GFP AAV, increased their preference for sucrose post-surgery (all comparisons between CRE-GFP GHSR null and other experimental groups, $p<0.05$ ). 
A

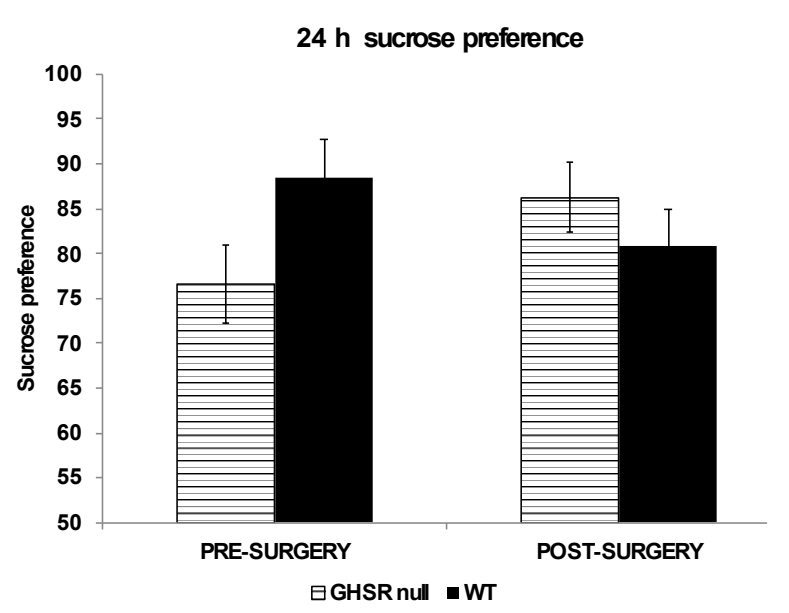

B

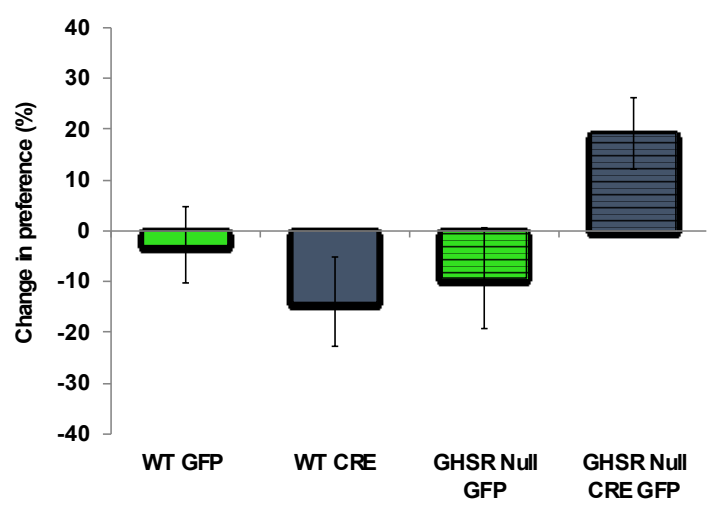

Figure 2. 24h sucrose preference. A) $24 \mathrm{~h}$ sucrose preference pre- and post-surgery. Prior to surgery, GHSR null mice preferred the $3.5 \%$ sucrose solution less than the WT mice. Post-surgery, there was no significant difference between GHSR null and WT mice. B) Change in preference from pre- to post-surgery. Only GHSR null CRE-GFP mice, given the AAV to reinstate GHSR transcription in the VTA, increased their preference for the sucrose solution 3 weeks following surgery. $(* p<0.05)$.

\section{Discussion.}

Utilizing two distinct genetic approaches, this series of studies demonstrates that a lack of functional GHSRs, whether via a point mutation which produces a truncated version of the GHSR in rats, or via transcriptional blockade of the receptor in mice, reduces a number of food reward behaviours involving sucrose reinforcers. In the rats, a lack of GHSR signaling reduced motivation for sucrose pellets without affecting appetite for regular, chocolate flavoured, or high-fat chocolate-flavoured pellets, and also reduced the ability of sucrose to facilitate a place preference in a CPP paradigm. In the mice lacking GHSR, preference for a 3.5\% sucrose solution was lower than the observed preference in WT mice, and reinstating GHSR in the VTA increased sucrose preference. Together these data are consistent with a role for GHSR signaling in sucrose preference and, moreover, suggest that GHSR signaling within the VTA is required to exhibit a strong preference for the sucrose solution. 
Consistent with our hypothesis, we found that the $\mathrm{GHSR}^{\mathrm{m} 1 / \mathrm{Mcwi}}$ were less motivated to obtain sucrose reinforcers in a PR operant task, reflecting reduced incentive salience of the sucrose and a subsequent reduction in motivation to obtain it. Somewhat surprisingly, we saw no effect on responses for the high-fat chocolate flavoured pellets, despite literature suggesting that similar types of reinforcers typically generate high levels of responding (Sharma et al., 2012), as well as that chronic ghrelin infusion into the VTA enhanced pressing for similar pellets (King et al., 2011). Moreover, the latter pellets have been shown to be effective in inducing persistent synaptic plasticity in the NAcc (Noye Tuplin et al., 2018). The use of a supraphysiological dose of ghrelin in our previous study (King et al., 2011) could underlie the enhanced responding beyond what typically occurs in a hungry rat. Alternatively, it is possible that some form of compensatory mechanism has developed in the GHSR ${ }^{\mathrm{m} 1 / \mathrm{Mcwi}}$ rats which enables them to respond fully to multiple palatable reinforcers. To complement the data generated under PR responding, during which the amount of effort expended by the animal is thought to reflect the potency of the reinforcer, we subsequently examined the preference for the same sucrose pellets in a CPP paradigm, during which rats display their preference for the reinforcer by spending more time in the environment with which it was previously (repeatedly) paired. Unlike PR tests, CPP allows for the examination of appetitive behaviour generated by food rewards in the absence of orosensory or post-ingestive feedback during the test itself.

Previous studies examining food reward behaviours using sucrose as a reinforcer have shown impaired initiation of sucrose consumption in response to peripheral injection of the ghrelin receptor antagonist D-Lys-growth hormone releasing peptide (D- 
Lys-GHRP-6) (Johnson et al., 2009), a decrease in consumption of a $2 \%$ sucrose solution in prairie voles (Stevenson et al., 2016), and decreased motivation to obtain sucrose in progressive ratio paradigms in mice (Skibicka et al., 2011a; Skibicka et al., 2012a; Terrill et al., 2018). Further, repeated intermittent access to sucrose has been shown to impair food-cue reward learning in rats, and repeated central administration of ghrelin was shown to mimic these effects, suggesting that changes in D2 dopamine receptors (D2DR) as a result of sucrose exposure, which are hypothesized to underlie impaired food-cue learning and enhance inappropriate eating habits, may be mediated by ghrelin (Sharpe et al., 2016).

In experiment 1.2, our finding that rats did not form a CPP under sated conditions are consistent with the findings of other investigators, in that non-food deprived animals will not form a CPP to a food reward (Figlewicz et al., 2001; Chuang et al., 2011). When rats were conditioned and tested in a fasted state, however, they spent more time in the sugar paired chamber, which is consistent with previous studies showing that FR bolsters the motivational status of the rodent by enhancing the incentive salience of the food reinforcer being offered, increasing the drive to obtain it (Sharma et al. 2012; van der Plasse et al., 2015), or spend time in a context associated with its delivery (Perello et al., 2010). Counter to our hypothesis, however, we did not find that there were differences in the amount of time spent in the sugar-paired chamber on this test day between the $\mathrm{GHSR}^{\mathrm{m} 1 / \mathrm{Mcwi}}$ and WT rats. This may reflect the fact alternate interoceptive signaling changes are induced by fasting, and these may augment the time spent in the sugar-paired chamber to facilitate procurement of the palatable food, even in the absence of ghrelin signaling. For example, decreased circulating leptin levels during fasting may contribute 
to a release of inhibition on the drive to obtain food reward, resulting in formation of a CPP to the sugar-paired side (Figlewicz et al., 2001), which could be a manifestation of the interaction of leptin with brain reward circuits (Fulton, Woodside, and Shizgal, 2000). In fact, in addition to the opposing effects ghrelin and leptin have in the arcuate nucleus of the hypothalamus (ARC), through direct modulation of subpopulations of neuropeptide-Y (NPY) and agouti-related protein (AgRP) neurons respectively, it appears that ghrelin and leptin also have opposing effects in terms of both the plasticity and activity of dopamine neurons in the VTA as well (Abizaid et al. 2006; Thomson and Borgland, 2013).

Prior studies have shown that when conditioned in a hungry state, but tested while satiated, rats will show place conditioning for food (Spyraki et al., 1982). The results from test day 3 which were carried out under these conditions confirmed these findings and showed that WT mice spent more time on the sugar-paired side than the rats without functional GHSR. The latter finding provides compelling evidence that associative learning is heavily influenced by the satiety state of the animal (i.e. learning to associate food cues with a particular context is enhanced while in a hungry state) and that preference for an environment in which contextual cues predict the presence of a palatable food does not necessarily require the animal to be hungry at the time of testing. This could be particularly relevant in the context of human dieting, if this is also true in humans. As dieters are frequently in a food restricted state, this may serve to enhance learned associations between food cues and availability of preferred foods, leading to the seeking out of those foods even while not hungry, similar to what we saw in our test day 3 results. The difference observed in time spent in the sugar-paired chamber between 
$\mathrm{GHSR}^{\mathrm{m} 1 / \mathrm{Mcwi}}$ and WT rats may be a consequence of a lack of ghrelin signaling through its receptor, combined with the decrease in circulating leptin, resulting in a reduced preference for the sugar-paired chamber. The site of action of leptin and ghrelin (in WT rats, or lack thereof in $\mathrm{GHSR}^{\mathrm{m} 1 / \mathrm{Mcwi}}$ ) in producing such differences is unknown, but substantial evidence points to a role for their action on brain reward circuits. In support of this hypothesis, we found genotypic differences in expression of the immediate early gene cFOS in the VTA, with WT rats showing greater activation following the final CPP test than GHSR ${ }^{\mathrm{m} 1 / \text { Mcwi }}$ rats. We also found differences in expression of $\mathrm{cFOS}$ in the MePV following CPP, which has been shown to have dense expression of GHSRs and may play a role in the expression of anxiety in rats. It is possible that the GHSR ${ }^{\mathrm{m} 1 / \mathrm{Mcwi}}$ rats are more anxious than the WT rats, which may prevent them from traversing into the brighter, albeit sugar-paired chamber. Previous data from our lab has also demonstrated greater anxiety in these rats during a CPP protocol for cookie dough (St.Onge, 2017).

In order to assess the role of GHSR signaling in the VTA on sucrose preference in a two-botte choice test, we took another approach, using mice that harbour a TBC upstream of the GHSR promoter region, obstructing transcription of GHSR mRNA. Removal of this TBA via adeno-associated viral vector delivery of CRE, is able to reinstate transcription of GHSR mRNA to varying degrees (see Study 1.3.2, Figure 1, and Study 2.3, Figure 1). In the current study, sucrose preference was lower in GHSR null mice relative to WT mice, prior to reinstatement of the GHSR, and these results provide further evidence that the presence of functional GHSRs in the VTA is important for the full expression of food-reward behaviours, particularly when sucrose is the reward of choice. Interestingly, differences in sucrose preference were seen in sated mice under 
conditions of ad libitum food intake (i.e. the mice were not hungry). This is somewhat in line with the CPP data from experiment 1.2 (although they were conditioned when hungry), in that, in the absence of hunger, we see differences between the GHSR null and WT animals in their preference for either an environment previously paired with sucrose, or a 3.5\% sucrose solution vs. water. The most exciting result of the final experiment was that reinstatement of GHSR expression in the VTA was sufficient to induce an increase in preference for the sucrose solution in GHSR null mice, such that 3 weeks after AAV rescue of the GHSR, there were no longer any differences in preference between the WT and GHSR null mice. This provides strong evidence that GHSR signaling in the VTA influences the consumption of a sucrose solution in mice. The exact mechanism by which GHSR signaling mediates this behaviour is yet to be discovered. Interactions with either dopaminergic or opioidergic circuity (or both) are very likely, as ghrelin and the GHSR are both shown to have direct influence on both systems at the level of the VTA.

\section{Conclusion.}

In the last decade in particular, sugar has been vilified in popular science and the media as being the primary cause of type II diabetes, obesity, and most recently, Alzheimer's disease. There is some evidence to support such claims, particularly as they are studied in rodent models, wherein repeated or excessive consumption of sugar is associated with altered processing of cues indicative of food reward (Sharpe et al., 2015). This could contribute to enhanced intake of sugar and other palatable foods, the subsequent metabolic and psychological consequences associated with loss of control over intake and ultimately, obesity. To gain a basic understanding of some of the factors that influence the rewarding properties of sucrose and what motivates an organism to obtain it, we used genetic manipulations in rats and mice to determine whether a lack of 
functional GHSRs reduced the reward value of sucrose. Our results would suggest that, indeed, GHSR signaling is important for the expression of reward derived from sucrose reinforcers, and that restoration of the receptor in a region important for reward derived from food, the VTA, is able to restore a robust preference for sucrose in mice that were previously less interested in the sucrose solution. This suggests that the VTA is a key neural substrate for sucrose reward, and that the GHSR in the VTA is important for sucrose preference. It will be interesting to determine in future studies if the motivation and place preference for sucrose are also mediated by GHSR specifically in the VTA. 
Table 1. Nutritional composition of pellets used for operant conditioning.

\begin{tabular}{|c|c|c|c|}
\hline Type of Pellet & Nutritional Content & $\%$ by Weight & $\mathrm{kcal} / \mathrm{g}$ \\
\hline \multirow[t]{4}{*}{ Grain-based purified } & Protein & 18.7 & 0.75 \\
\hline & Carbohydrates & 5.6 & 2.36 \\
\hline & Fat & 6.5 & 0.50 \\
\hline & \multicolumn{2}{|l|}{ Total } & 3.61 \\
\hline \multirow[t]{4}{*}{ Chocolate flavored } & Protein & 18.4 & 0.74 \\
\hline & Carbohydrates & 59.1 & 2.36 \\
\hline & Fat & 5.5 & 0.50 \\
\hline & \multicolumn{2}{|l|}{ Total } & 3.60 \\
\hline \multirow[t]{4}{*}{ Sugar } & Protein & 0 & 0.00 \\
\hline & Carbohydrates & 89.5 & 3.79 \\
\hline & Fat & 0 & 0.05 \\
\hline & \multicolumn{2}{|l|}{ Total } & 3.84 \\
\hline \multirow[t]{4}{*}{ High-fat chocolate flavored } & Protein & 10.2 & 0.41 \\
\hline & Carbohydrates & 42.8 & 1.71 \\
\hline & Fat & 35.1 & 3.16 \\
\hline & \multicolumn{2}{|l|}{ Total } & 5.28 \\
\hline
\end{tabular}


Table 2. Primer sequences for Study 1.3 and 2.3.

\begin{tabular}{|l|l|c|}
\hline \multicolumn{1}{|c|}{ Gene } & Forward & Reverse \\
\hline $\begin{array}{l}\text { B-actin } \\
\text { mouse }\end{array}$ & GAACCCTAAGGCCAACCGTG & GGTACGACCAGAGGCATACAG \\
\hline $\begin{array}{l}\text { GHSR-1a } \\
\text { mouse }\end{array}$ & CTCAGGGACCAGAACCACAAAC & ACAAAGGACACCAGGTTGCAG \\
\hline
\end{tabular}


Appendix A. Transgenic construct of mice from University of Texas Southwestern.
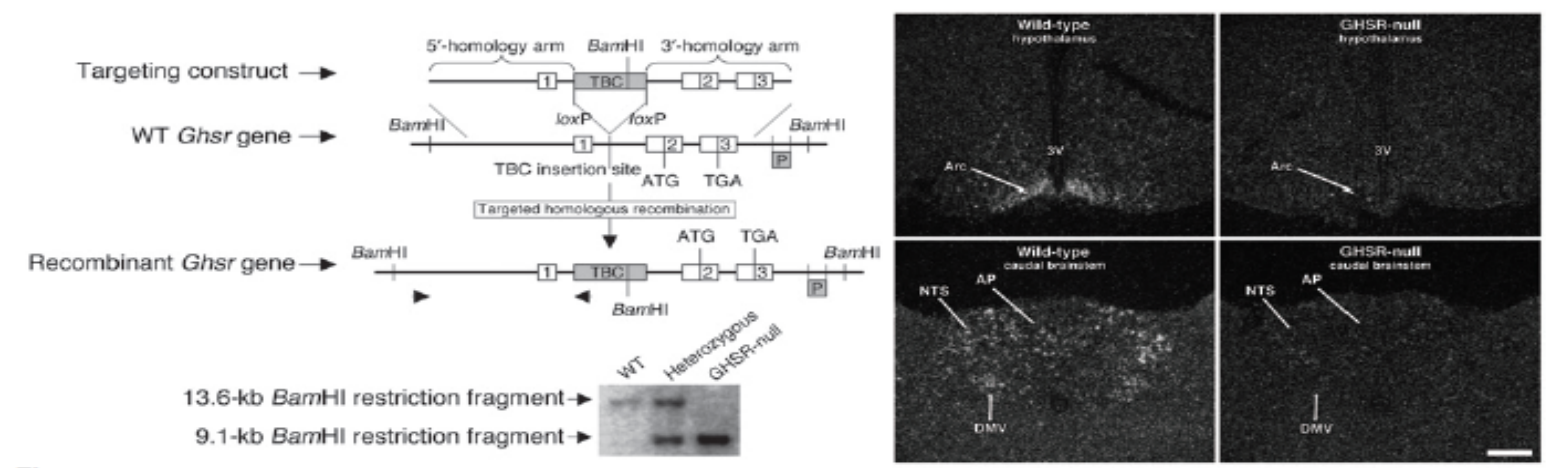

Figure 1

Gene targeting of the Ghsr locus. Upper left panel: Schematic diagram of the derivation of GHSR-null mice by homologous recombination. Lower left panel: Southem blot analysis of genomic DNA extracted from representative progeny of mating animals heterozygous for the recombinant Ghsr allele. Right panels: Representative dark-field photomicrographs of in situ hybridization histochemistry experiments performed on mouse brains using a mouse GHSR-specific riboprobe. Ghsr mRNA expression is evidenced by the white-appearing silver granules. 1-3, Ghsr exons 1-3; 3V, third ventricle; AP, area postrema; Arc, arcuate nucleus; DMV, dorsal motor nucleus of the vagus; NTS, nucleus of the solitary tract; P, Southern probe. Scale bar: $200 \mu \mathrm{m}$ (applies to all 4 panels).

From: Zigman, J.M., Nakano, Y., Coppari, R., Balthasar, N., Marcus, J.N., Lee, C.E., Jones, J.E., Deysher, A.E., Waxman, A.R., White, R.D., Williams, T.D., Lachey, J.L., Seeley, R.J., Lowell, B.B., and Elmquist, J.K. (2005). Mice lacking ghrelin receptors resist the development of diet-induced obesity. JCI, 115(12), 3564-3572. 
Appendix B. Conditioned place preference (CPP) apparatus and food pairing.

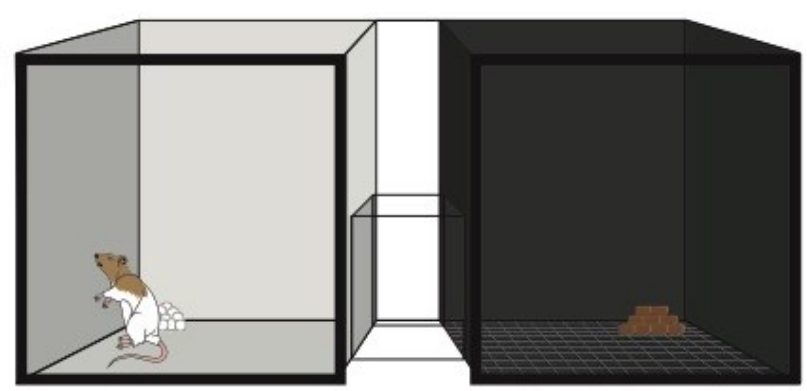




\section{Chapter 3: Investigation of a role for ghrelin signaling in binge-like feeding in mice under limited access to high-fat diet (Studies 2.1, 2.2).}

Citation: King, S.J., Rodrigues, T., Watts, A., Murray, E., Wilson, A. and Abizaid, A. (2016). Investigation of a role for ghrelin signaling in binge-like feeding in mice under limited access to high-fat diet. Neuroscience, 319, 233-245.

Abstract. Binge eating is defined by the consumption of an excessive amount of food in a short time, reflecting a form of hedonic eating that is not necessarily motivated by caloric need. Foods consumed during a binge are also often high in fat and/or sugar. Ghrelin, signaling centrally via the growth-hormone secretagogue (GHSR), stimulates growth hormone release and appetite. GHSR signaling also enhances the rewarding value of palatable foods and increases motivation for such foods. As ghrelin interacts directly with dopaminergic reward circuitry, shown to be involved in binge eating, the current studies explored the role of GHSR signaling in a limited access model of binge eating in mice. In this model, mice received either intermittent (INT) or daily (DAILY) access to a nutritionally complete high-fat diet (HFD) for 2-h late in the light cycle, alongside 24-h ad libitum chow. In CD 1 mice, 2-h exposure to HFD generated substantial binge-like intake of HFD, as well as a binge-compensate pattern of 24-h daily intake. INT and DAILY groups did not differ in 2-h HFD consumption, while INT mice maintained stable intake of chow despite access to HFD. GHSR knock-out (KO) and wild-type (WT) mice both binged during HFD access and exhibited the same binge-compensate pattern. INT GHSR KO mice did not binge as much as WT, while DAILY KO and WT were comparable. Overall, GHSR KO mice consumed fewer calories from HFD, regardless of access condition. GHSR KO mice also had reduced activation of the nucleus accumbens 
shell, but not core, following HFD consumption. These data support the ability of INT HFD in mice to induce a binge-compensate paper of intake that emulates select components of binge eating in humans. There also appears to be a role for GHSR signaling in driving HFD consumption under these conditions, potentially via mediation of reward-related circuitry.

\section{Introduction.}

Binge eating is defined by the consumption of an excessive amount of food in a short time frame and is often associated with a sense of loss of control over the ability to cease eating once nutritional needs have been met (APA, 2013). Binge eating, therefore, reflects a form of hedonic eating that is not motivated by the necessity for calories or specific macronutrients, and is likely to be regulated in part by mesolimbic reward circuitry (Davis et al., 2009; for a review, see Bello and Hajnal, 2010). In support of this view, it is most often highly-palatable and energy- dense foods that are consumed during a binge episode (Kales, 1990; Elmore and DiCastro, 1991), and consumption of such foods are known to influence the short- and long-term plasticity of both homeostatic and brain reward circuitry (Bello et al., 2009; Johnson and Kenny, 2010). The precise mechanisms underlying the eating behavior seen in individuals that binge eat remain poorly understood. Identifying the factors that contribute to binge eating in both normal weight and obese binge eating disorder (BED) models, components of which may be dissociable, will aid in the understanding of the disorder and ideally improve treatment options for those that binge eat.

A variety of animal models of binge-type eating have been developed in recent decades (Corwin et al., 1998; Boggiano et al., 2007; Berner et al., 2008; Bello et al., 2009; Lardeux et al., 2013; Bake et al., 2014a). The limited access model, first 
demonstrated in rats by Corwin et al. (1998), is used to elicit binge-like consumption and alter patterns of fat intake that emulate select components of eating behaviors seen in humans with BED. This model is based on findings which show that restricting access to a substance can reliably enhance intake when that substance again becomes available (Wayner and Fraley, 1972; Pinel and Huang, 1976; Corrigall and Coen, 1989), and is thought to partially underlie the repeated failures of dieters who attempt to self-restrict intake of energy dense foods. In this model, rats placed under time restricted and sporadic access to fat demonstrate a binge-compensate pattern of feeding, such that they consume a greater number of calories on access days, and a reduced number of calories on nonaccess days relative to the control group who do not receive fat (Corwin et al., 1998). While this model does not accelerate the development of obesity in all studies (Czyzyk, Sahr and Stanick, 2010; Corwin et al., 1998; Dimitrou et al., 2000; Corwin, 2004) it can alter patterns of intake and dietary composition, which may be reflected in changes in endocrine function and central nervous system activation.

Recent studies examining the impact of intermittent (INT) but regularly scheduled, and therefore predictable, access to high-fat diet (HFD) found that rats with INT access exhibit a number of metabolic consequences, such as impaired glucose tolerance, increased insulin levels and increased fat mass, relative to controls (Bake et al., 2014b). Interestingly, the lack of alterations in both orexigenic and anorexigenic peptide gene expression in the arcuate nucleus of the hypothalamus (ARC) prior to scheduled HFD or sucrose access in mice and rats, suggests that alternate mechanisms may be involved in the regulation of such binge-eating behavior (Bake et al., 2013). The excessive intake under such access conditions may be driven by reward systems that 
supplant the hypothalamic homeostatic control of feeding. Rats allowed INT scheduled access to a sweet-fat mixture not only consumed more food, but also exhibited higher terminal ghrelin levels as compared to rats with continuous access (Bello et al., 2009), suggesting that ghrelin signaling may play a role in increasing consumption of the palatable food when access is restricted. Elevated ghrelin levels have also been found in rats that developed binge-like eating when exposed to chow followed by a sweet-fat mixture subsequent to $2 \mathrm{~h}$ of daily food deprivation (Cottone et al., 2008).

Ghrelin is a 28 -amino acid peptide that is produced primarily by endocrine mucosal cells in the stomach (Kojima et al., 1999; Date et al., 2000) and its predominant functions include the stimulation of growth hormone release and the regulation of food intake and energy balance, both in the short- and long-term (Cummings, 2006). Ghrelin plays a role in meal initiation by peaking just prior to a meal in schedule fed humans and rodents (Cummings et al., 2001; Drazen et al., 2006). Elevated levels of ghrelin also result in a greater accumulation of body fat stores and a concomitant reduction in physical activity, leading to increased weight gain and reduced basal metabolic rate (Tschop et al., 2000). Ghrelin binds to the growth-hormone secretagogue receptor of the 1A subtype (GHSR; Howard et al., 1996), present at appreciable levels in widespread regions of the rat and mouse brain as detected by in situ hybridization (Guan et al., 1997; Zigman et al., 2006). Outside of ghrelin's actions on homeostatic nuclei in the ARC (for review see Horvath et al., 2001; Abizaid and Horvath, 2008), ghrelin exerts a substantial influence on reward circuitry (for review see: Abizaid and Horvath, 2008; Abizaid, 2009; Dickson et al., 2011; Skibicka and Dickson, 2011a, Perello and Dickson, 2015). Both systemic and direct ventral tegmental area (VTA) ghrelin administration enhances the 
release of dopamine (DA) in the nucleus accumbens (NAcc) (Abizaid et al., 2006;

Jerlhag et al., 2006, 2007) and increases excitatory inputs onto VTA DA producing cells that co-localize with GHSR (Abizaid et al., 2006). Ghrelin administration also increases the rewarding value of highly palatable food (Egecioglu et al., 2010; Perello et al., 2010) as well as the motivation to work for such foods (Skibicka et al., 2011b, 2012; King et al., 2011). Given the ability of ghrelin to interact directly with dopaminergic reward circuitry known to be involved in binge eating, it could potentially be involved in the development of binge eating behaviors generated by INT access to highly palatable foods in mice.

To date, the role of ghrelin in binge eating in humans has been equivocal. An early study implicated the presence of the Leu72Met variant of the ghrelin gene in the development of BED in a small cohort of human subjects (Monteleone et al., 2007). Additional studies have reported that plasma ghrelin is reduced in obese- BED individuals (Geliebter et al., 2005; Monteleone et al., 2005) as well as in those with a higher frequency and severity of binge-purge behaviors (Troisi et al., 2005). Interestingly, the post-prandial suppression of ghrelin is also attenuated in obese-BED populations (Geliebter et al., 2005), which may reduce the satiating effect of a meal and may contribute to over-eating in the short term. It is currently unknown whether ghrelin signaling in normal-weight populations of humans or rodents is important for the development of binge eating on high-fat foods.

We initially set out to establish whether the limited access model utilized in rats (Corwin et al., 1998; Davis et al., 2007) would be reproducible in mice using a nutritionally complete HFD. In Experiment 2.1, we hypothesized that subjecting adult male mice to a limited access model of HFD, allowing access for $2 \mathrm{~h}$ per day 3 times a 
week, would elicit a binge/compensate pattern of caloric intake, relative to both mice fed an HFD for $2 \mathrm{~h}$ daily and chow-fed controls. Given ghrelin's central role in driving food intake and enhancing food reward, in our second experiment we hypothesized that mice lacking intact ghrelin receptors (GHSR-KO) would exhibit an attenuated binge-like pattern of eating typically demonstrated under this access schedule in wild-type (WT) rodents. We also examined differences between the mice in terms of neuronal activation in reward-related brain regions using c-Fos immunohistochemistry.

\section{Experimental Procedures.}

Animals.

Experiment 2.1.

Twenty adult male CD-1 mice (Charles River, St. Constant, QC) weighing 35-45 grams were housed individually in a temperature and humidity controlled vivarium with a 12:12 light/dark schedule (Lights on: 07:00 to 19:00). All mice had ad libitum access to water and nutritionally complete standard laboratory chow (Teklad Global 14\% Protein Rodent Maintenance Diet 2014, Harlan Laboratories, Madison, WI, USA; percent of kilocalories derived from fat: 13 , protein: 20 , carbohydrates: $67 ; 2.9 \mathrm{kcal} / \mathrm{g}$ ) for the duration of the study. After one week of acclimatization to the vivarium environment, bodyweight and food intake were measured for 7 days to serve as a baseline. At the end of the baseline period, mice were given access to a nutritionally complete HFD overnight (Open Source Diets, D12492; Research Diets, Inc., New Brunswick, NJ; percent of kilocalories from fat: 60 , protein: 20, carbohydrates: $20 ; 5.24 \mathrm{kcal} / \mathrm{g})$. Mice were exposed to HFD overnight to prevent neophobia toward the diet, as well as to eliminate any mice that did not consume any HFD during the overnight exposure period. Two groups were 
matched by body weight and overnight HFD consumption and assigned to either (1) DAILY $(n=9)$; or (2) INT $(n=10)$ access to HFD for the remainder of the experiment.

\section{Experiment 2.2.}

To determine whether a lack of GHSR signaling would either hinder the development or reduce the magnitude of binge-like consumption of a HFD under an INT access schedule, we used mice sustaining an in-frame deletion of the GHSR gene which was replaced with a LacZ reporter gene $(\mathrm{n}=18$; GHSR KO), and their WT littermates $(\mathrm{n}=$ 18; GHSR +/+), originally crossed onto a C57BL/J6 DBA background, and subsequently backcrossed onto a C57BL/J6 background strain. Mice were originally obtained from a colony at Yale University as a kind gift from Dr. Tamas Horvath and derived from founder strains created at Regeneron Pharmaceuticals (Tarrytown, NY, USA). Baseline measurements and overnight HFD exposure were conducted as outlined in Experiment 1. However, two-thirds of the mice matched on 3-day chow consumption and overnight HFD intake were subsequently divided into 4 experimental groups: (1) KO DAILY and (2) WT DAILY, given access to the HFD for $2 \mathrm{~h}$ beginning at 15:30 each afternoon; (3) KO INT and (4) WT INT, given access to HFD for $2 \mathrm{~h}$ beginning at the same time as in the DAILY group, but only on Monday, Wednesday and Friday (M,W,F). The remaining mice formed two control groups, receiving HFD only during the initial overnight exposure period, but strictly ad libitum chow over the course of the experiment (1) Control KO $(n=6)$ and (2) Control WT $(n=4)$. All procedures were approved by the Canadian Council on Animal Care (CCAC) and the Carleton University Animal Care committee. 


\section{Limited access model.}

Mice were allowed ad libitum access to standard chow throughout the course of the experiment. Food intake and body weight were measured daily. For four weeks (Experiment 2.1) or six weeks (Experiment 2.2), mice were given either daily access to the HFD (DAILY) composed of 60\% kilocalories (kcal) from fat for two hours beginning at 15:30, or were given access to the diet only 3 times per week, (INT; MWF) at the same time of day. HFD was placed in small glass flat-bottomed bowls in the home cage of the mice on the days that they were scheduled to receive the HFD. The amount of HFD consumed during the 2-h period was recorded. At the time of HFD removal, the bottom of the cage was examined to ensure that no spillage was left unrecorded, potentially inflating KCAL intake during this period. We used a 4-week paradigm in Experiment 1 to determine if this length of exposure would be sufficient to induce binge-like feeding in mice. In designing Experiment 2, we hypothesized that GHSR KO mice may binge less than WT within a 4-week period, but that given the opportunity, may eventually match intake seen in WT, and therefore Experiment 2 was designed to be 6-weeks in length.

Experiment 2.2.

Immunohistochemistry.

One hour following their last exposure to HFD, mice were injected with a lethal dose of Dorminal (1 mg/kg i.p.; CDMV, Quebec, Canada) and perfused transcardially with $0.9 \%$ saline, followed by $4 \%$ paraformaldehyde. Brains were then extracted and post- fixed in $4 \%$ paraformaldehyde for $24 \mathrm{~h}$, followed by submersion in a $30 \%$ sucrose solution (w/v) prior to sectioning for cryoprotection. Forty micrometer sections containing the NAcc core and shell regions (Figs 18-22, Paxinos and Franklin, 2001) 
were sliced at $21 \mathrm{C}$ on a Thermoscientific ${ }^{\mathrm{TM}}$ Cryostat. Sections were placed into wells containing $0.1 \mathrm{M}$ phosphate buffer and every sixth section was subsequently processed for C-fos IR using a standard immunohistochemical detection protocol as has been outlined previously (Abizaid et al., 2005). Briefly, free-floating sections were washed in 0.1M phosphate buffer ( $\mathrm{PB}, \mathrm{pH} 7.4$ ), followed by incubation in a quenching solution (PB and $1 \% \mathrm{H}_{2} \mathrm{O}_{2}$ ) at room temperature (RT) for 30 minutes. Sections were then rinsed in $\mathrm{PB}$ and incubated at RT for $1 \mathrm{~h}$ in a blocking solution comprised of $0.3 \%$ Triton-X and bovine serum albumin (BSA) in PB. Sections were then incubated for $48 \mathrm{~h}$ at $4^{\circ} \mathrm{C}$ in a FOS-antirabbit primary antibody (Oncogene Science, Boston, MA, USA; 1:20,000) solution containing Triton-X and BSA. Sections were then rinsed in PB and incubated in a biotinylated IgG secondary antibody (donkey anti-rabbit; Jackson Immunoresearch Laboratories Inc., Westgrove, PA, USA; 1:250) for 1h, followed by an avidin-horseradish peroxidase complex (ABC Elite Kit, Vector laboratories, Burlington, ON, Canada; 1:500) and reacted with $0.05 \%$ diaminobenzidine and $0.03 \% \mathrm{H}_{2} \mathrm{O}_{2}$ (DAB, Sigma Aldrich, St. Louis, MI, USA) for 10 minutes, resulting in a dark brown staining of C-fos-positive nuclei. Sections were then mounted on gel- coated slides, dehydrated in a series of graded alcohol concentrations and submersed in Clearene (Surgipath). Slides were then coverslipped using Permount (Fisher Scientific, Ottawa, ON, Canada). A control group of mice not receiving HFD throughout the experiment served as a comparison group.

Two to three coronal sections obtained from each mouse were examined to quantify C-fos IR. All photos were taken under a 10x objective lens using standard bright-field microscopy on an Olympus BX51 microscope (Olympus Canada, Markham, ON, Canada) attached to an Olympus (DVC 2000C) camera, using Stereo Investigator 8 
software (MBF Bioscience, Williston, Vermont, USA). The quantification of C-fos IR was conducted using the Image J64 software (NIH freeware). C-fos positive cells were counted bilaterally in the NAcc. The counts reported are the mean ( \pm standard error of the mean (SEM)) percent of control mice for each group.

\section{Statistical Analyses.}

Experiment 2.1. To examine the patterns of intake across time, food intake data were analyzed using a one-way repeated measures ANOVA (day x access group) followed by Bonferroni corrected post-hoc tests, where omnibus differences were found. Independent sample t-tests were conducted on data examining overall means.

Experiment 2.2. Data were analyzed using two-way repeated measures ANOVA using day of study as the within-subjects factor, and genotype and access schedule as the between group factors. Follow up multiple comparisons using the Bonferroni correction were performed. Two-way ANOVAs (genotype x access group) on overall average data were conducted where outlined, and Bonferroni corrections were used to determine where differences between groups were present. Data from both experiments are expressed as mean \pm standard error of the mean (SEM). All statistical analyses were performed with SPSS 20.0.0 for Macintosh (IBM Corp., Armok, NY) and statistical significance was set at $\alpha=0.05$.

Results.

Experiment 2.1.

High fat diet (Figure $1 A$ and $B$ ).

The total number of calories consumed from HFD during the 2 hour access period did not differ between DAILY and INT groups on any day of the experiment (day $\mathrm{x}$ 
access group: $\left.F_{(12,204)}=.826, p>0.05\right)$. However, a significant main effect of day $\left(F_{(1,17)}\right.$ $=12.356, p<0.05)$ demonstrated that all mice increased the average amount of HFD that they consumed across the study period, starting at $5.5 \pm .5 \mathrm{kcal}$ on the first access day and increasing to $14.3 \pm 1.1 \mathrm{kcal}$ by the last access day. The overall average number of kcal consumed during the access period was equal to $10.9 \pm 1.1$ for the DAILY mice and 12.1 \pm 0.7 for the INT mice $\left(t_{(17)}=0.436, p>0.05\right)$, which in both cases came exclusively from HFD as the mice ate negligible amounts of chow during this period. While there were no group differences in HFD diet intake during the access period, CD-1 mice given either DAILY or INT HFD access schedules both exhibited binge-like eating behavior during the 2-hour exposure to HFD late in the light cycle. Mice in the INT group consumed 85 $\% \pm 6 \%$ of their average daily baseline intake from HFD whereas the DAILY mice consumed $71 \% \pm 7 \%$ within the same time period, however, the difference between groups was not statistically significant $\left(t_{(17)}=1.375, p>0.05\right.$, see Figure 1C).

Standard chow (Figure 1C).

In the 22 hour period following access to the HFD, we found that the INT group consumed a significantly greater amount of calories from standard chow, relative to the DAILY group (day $\mathrm{x}$ access group, $\left.F_{(12,204)}=9.994, \mathrm{p}<0.05\right)$. Post-hoc comparisons revealed that this difference emerged on the second access day and continued for the remainder of the access days ( $p<0.05$ for access days 2 through 13).

Total daily caloric intake (Figure 1D).

Total 24h intake was significantly different between groups on both access days $\left(t_{(17)}=10.140, p<0.05\right)$, upon which the INT group consumed a greater number of calories than DAILY mice (28.0 \pm .6 vs. $20.1 \pm .5)$, and on non-access days $\left(t_{(17)}=9.871\right.$, 
$p<0.05)$, upon which INT mice consumed fewer calories than DAILY mice $(10.1 \pm .5$

vs. $17.4 \pm .5)$.

Change from baseline daily intake (Figure $1 E$ and $F$ ).

To determine if total daily intakes differed from average baseline intake, we calculated a change from baseline score for every day of the study. A two-way repeated measures ANOVA (day $\mathrm{x}$ group) demonstrated that mice consumed differing amounts of kcal (relative to their baseline) across access days, depending on their access condition (day $\mathrm{x}$ group interaction: $F_{(12,204)}=4.235, p<0.05$ ). On average, mice with INT access to HFD ate $11.2 \pm 0.9 \mathrm{kcal}$ whereas the group with DAILY access ate merely $2.6 \pm 0.9 \mathrm{kcal}$ above their average baseline intake on access days $\left(F_{(1,17)}=41.887, p<0.05\right)$. With the exception of the first HFD access day, the INT group had higher intakes than the DAILY group on all access days $(p<0.05)$. Across non-access days, the INT group reduced their caloric intake to a greater extent than the DAILY group relative to their baseline (day $\mathrm{x}$ group interaction: $\left.F_{(15,255)}=4.100, p<0.05\right)$, and differences between groups were significant on non-access days $1,3,4,6,7,8,9,11,12,13,14,15$, and $16(p<0.05)$. Therefore, restricting access of the HFD for two hours to only three days per week (M,W,F) induced a overeat-compensate pattern of consumption in the INT mice. Unexpectedly, this was not due solely to excessive consumption of HFD during the $2 \mathrm{~h}$ access period on the part on the INT mice, as both groups consumed binge-like amounts of HFD in 2 hours. Rather, differences between groups appeared to emerge as a result of the INT mice showing greater chow consumption on HFD access days (see Figure 1C). The overall mean change from baseline data for both access and non-access days can be seen in Figure 1D. Both DAILY and INT groups increased caloric intake on days when they had access to HFD, relative to their baseline, but INT mice increased intake to a significantly 
greater extent than did DAILY mice $\left(t_{(17)}=6.472, p<0.05\right)$. On non-access days, DAILY mice maintained a level of intake similar to baseline $(1.1 \pm .7 \mathrm{kcal})$, but the INT group reduced their intake $\left(-5.1 \pm .7 \mathrm{kcal} ; t_{(17)}=6.034, p<0.05\right)$ likely due to increased caloric consumption on the previous days.

Cumulative intake (Figure $1 G$ ).

We found that mice in the INT group exhibited greater cumulative 24-hour caloric intake (comprised of both diets) across HFD access days, relative to the DAILY group (day $\mathrm{x}$ access group: $F_{(12,204)}=89.53, p<0.05$ ). Post-hoc tests revealed that significant differences emerged on the second access day and persisted throughout the course of the experiment ( $p<0.05$ on all days). By the end of the experiment, INT mice had eaten 336.6 \pm 7.3 kilocalories on access days, whereas DAILY mice had consumed $244.8 \pm 6.5$ kcal on the same days.

\section{Diet Composition (Figure 1H).}

Over the course of the experiment, the INT group consumed a greater percentage of their daily calories from standard chow $(69.4 \pm 4.1)$ compared to the DAILY group $\left(39.2 \pm 4.2 ; F_{(1,18)}=27.5, \mathrm{p}<0.05\right)$. The opposite pattern was found in the DAILY group, who consumed a greater percentage of their calories from HFD $(60.8 \pm 4.2)$ than the INT group $(30.6 \pm 4.1)$. That the group with a greater frequency of access to HFD consumed a greater percentage of their overall calories from HFD is line with previously reported results (Corwin et al., 1998), even though the animals in the INT group also demonstrated binge-like consumption during HFD exposure. Despite consistently bingeing during the short exposure window 3 days a week, the INT animals did not consume as many overall calories from HFD as those that received the HFD every day for the same amount of time. 
Body Weight (Figure 1I).

Body weights did not differ between DAILY and INT mice fed high-fat diet upon completion of the experiment $\left(\mathrm{t}_{(17)}=1.895, \mathrm{p}>0.05\right)$.
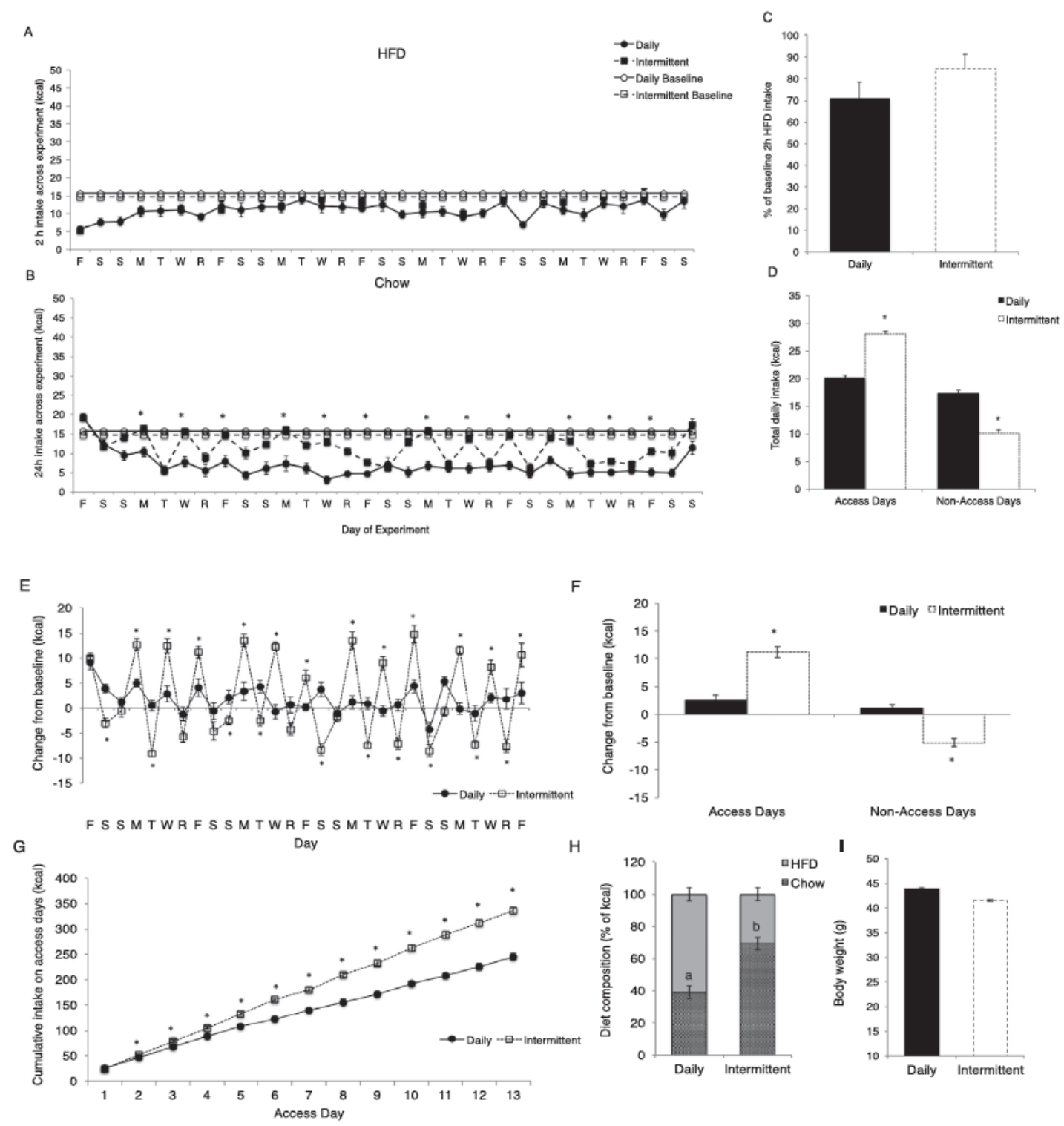

Figure 1 A-I. Feeding patterns, diet selection and body weight in CD-1 mice. Caloric intake from HFD ( $2 \mathrm{~h}$ ) across the experiment (A). Intake of HFD on access days (M,W,F) did not differ between DAILY and INT mice, however, INT mice show greater chow intake within the same 24 hour period. Percent of baseline intake consumed during $2 \mathrm{~h}$ of HFD access (B). All mice consumed $\geq 71 \%$ of their average daily intake within 2 hours. Caloric intake from chow across the experiment showing enhanced intake in INT mice on access days $\left(\mathrm{C} ;{ }^{*} \mathrm{p}<0.05\right)$. Total daily intake $(24 \mathrm{~h} \mathrm{kcal})$ was higher in INT mice than in DAILY mice on access days and lower than DAILY mice on non-access days (D, 
$\left.{ }^{*} \mathrm{p}<0.05\right)$. Change from baseline kcal across the experiment $(\mathrm{E}, * \mathrm{p}<0.05)$, and average change from baseline intake on access and non-access days $\left(\mathrm{F},{ }^{*} \mathrm{p}<0.05\right)$. INT mice consumed a significantly greater amount of calories on days that they received access to HFD. They also reduced their intake relative to baseline on days following access to HFD, demonstrating a binge-compensate pattern of intake. DAILY mice did not significantly over- or under-consume relative to baseline on either access or non-access days.

Cumulative $24 \mathrm{~h}$ intake on HFD access days (G). Mice in the INT group consumed a greater cumulative number of kilocalories on these days, relative to the DAILY mice. Statistically significant differences were found beginning on Day 2 of access and persisted over the course of the experiment. $\left({ }^{*} p<0.05\right)$. Percentage of kilocalories over the course of the experiment derived from either HFD or standard chow $(\mathrm{H})$. Mice in the DAILY group consumed a greater percentage of their kilocalories from HFD than from chow, whereas mice in the INT group consumed a greater percentage from standard chow $(a, b, p<0.05)$. Body weight at the end of the experiment (I).

\section{Experiment 2. 2.}

High Fat Diet. (Figure $2 A$ and $B, G$ ).

GHSR KO and WT mice demonstrated differential patterns of intake when placed

under the different schedules of HFD access. Two-way repeated measures ANOVA

revealed that there was no effect of day over the course of the experiment, suggesting that HFD intake was stable across the 6-week period. However, significant main effects of both access $\left(F_{(1,23)}=11.574, p<0.05\right)$ and genotype $\left(F_{(1,23)}=13.076, p<0.05\right)$ were present. KO mice ate less of the HFD than did WT mice $(6.1 \pm .4 \mathrm{kcal}$ vs. $7.9 \pm .4 \mathrm{kcal}$, respectively) and DAILY MICE ate more HFD than INT mice $(7.9 \pm .4$ kcal vs. $6.1 \pm .4$ kcal, respectively; see Figure 2A). Two-way ANOVA (access x genotype) for overall average HFD kcal consumption on access days revealed similar results, with main effects of both genotype $\left(F_{(1,27)}=16.211, p<0.05\right)$ and access $\left(F_{(1,27)}=8.6753, p<0.05\right) . \mathrm{KO}$ mice consumed less of the HFD during access relative to WT mice, and DAILY mice consumed a greater amount of HFD than INT mice. However, a significant interaction between access schedule and genotype was also found $\left(F_{(1,27)}=5.461, p<0.05\right)$, suggesting that the effect of genotype on HFD intake is dependent on access schedule. 
While both KO and WT mice receiving DAILY HFD ate a similar amount of HFD during the access period ( $p>0.05)$, KO mice receiving HFD on an INT schedule failed to increase their intake of HFD to the extent seen in WT mice $(p<0.05)$, see Figure 2B.

As in Experiment 2.1, placing mice under an INT or DAILY schedule of HFD access induced binge-like consumption during the HFD access period. Two-way ANOVA examining the mean percentage of control group kcal consumed from HFD during the 2 hour HFD access revealed a main effect of genotype $\left(F_{(1,23)}=7.236, p<\right.$ 0.05), with WT mice consuming a greater percentage of daily control intake from HFD $(50.1 \% \pm 3.4 \%)$ as compared to $\mathrm{KO}$ mice $(35.4 \% \pm 3.5 \%$, see Figure $2 \mathrm{G})$. Main effect of access was also significant $\left(F_{(1,23)}=11.499, p>0.05\right)$ with DAILY mice $(53.9 \% \pm 2.5 \%)$ consuming more than INT mice $(41.9 \% \pm 2.5 \%)$. A marginal interaction $\left(F_{(1,23)}=4.129, p\right.$ $=0.054)$ suggested a trend, with $\mathrm{KO}$ mice that received HFD intermittently not bingeing to the same extent as the rest of the groups, and this likely to contributes to the main effect of access, resulting in lower intake in mice receiving INT access to fat.

Standard Chow (Figure 2C and D).

A two-way repeated measures ANOVA for kcal derived from chow across access days revealed a day $\mathrm{x}$ access interaction $\left(F_{(34,510)}=2.235, p<0.06\right)$, such that both INT and DAILY groups reduced their chow intake over the course of the experiment. A main effect of access was also found $\left(F_{(2,30)}=15.307, p<0.05\right)$, with INT and DAILY mice both consuming less chow than control mice (see Figure $2 \mathrm{C}$; M,W and F). Overall average chow intake on access days also differed between groups. A main effect of access $\left(F_{(2,36)}=17.989, p<0.05\right)$ was revealed by two-way ANOVA (genotype $\mathrm{x}$ access schedule). Both DAILY $(p=0.000)$ and INT $(p=0.001)$ mice consumed less chow than the control group, as control mice did not receive HFD and therefore calories were 
derived exclusively from chow. While DAILY mice consumed modestly less chow than the INT mice on access days $(9.4 \pm .6 \mathrm{kcal}$ vs. $11.4 \pm .6 \mathrm{kcal})$, this difference was not statistically significant (see Figure 2D).

Two-way repeated measures ANOVA on non-access days revealed a day $\mathrm{x}$ access interaction $\left(F_{(2,660)}=2.214, p<0.05\right)$, showing that DAILY mice reduced their chow intake on non-access days across the experiment, although these were not technically non-access days for this particular group as they were still receiving fat on these days. There was also a main effect of access $\left(F_{(2,30)}=17.76, p<0.05\right)$. On most non-access days, both control and INT groups were consuming more chow than the DAILY groups (see Figure 2C; T,R,S and S).

Change from baseline caloric intake (Figure $2 E$ and $F$ ).

To examine whether the DAILY and INT access conditions induced changes in overall daily food consumption relative to the control chow-only group, we calculated the change from baseline kilocalories that all access groups consumed over a $24 \mathrm{~h}$ period (kcal consumed on access days-average baseline $\mathrm{kcal}$ ). The means were calculated separately for days on which the mice had access to a HFD and for days on which they had access exclusively to chow (i.e. non-access days; only occurred within the INT and control groups). Within the DAILY group, consumption on alternating access and nonaccess days should not differ, theoretically, since these days are comprised of identical timing and food-related cues. On access days, there was a significant main effect of access condition $\left(F_{(2,35)}=3.956, p<0.05\right)$. Mice receiving INT access consumed a greater number of calories above their average baseline intake $(2.9+/-0.5)$ compared to the control groups $(0.7+/-0.6)$. DAILY mice consumed an intermediate number of calories 
above baseline $(1.3+/-0.6)$, but did not differ from either the control $(p>0.05)$ or INT groups $(p>0.05)$. There was no main effect of genotype $\left(F_{(1,35)}=9.66, p>0.05\right)$, nor a significant interaction $\left(F_{(2,35)}=0.324, p>0.05\right)$.

On non-access days, there was a main effect of access condition $\left(F_{(2,35)}=6.314, p\right.$ $<0.05)$, wherein the INT groups reduced kcal intake to a greater extent than both control (INT: $-2.1 \pm 0.5$ vs. Controls: $-0.1 \pm 0.6, p<0.05)$ and DAILY groups $(0.2 \pm 0.5, p<$ $0.05)$. There was no significant main effect of genotype $\left(F_{(2,35)}=3.627, p>0.05\right)$, nor a significant interaction between genotype and access condition for $24 \mathrm{~h}$ intake $\left(F_{(2,35)}=\right.$ $1.096, p>0.05)$.

24 daily intake on access and non-access days (Figure 2H).

Two way ANOVA examining average total $24 \mathrm{~h}$ intake on access days revealed a main effect of access $\left(F_{(2,35)}=6.065, p<0.05\right)$. Both DAILY and INT mice consumed more on access days than the control group. There was also a marginal interaction $\left(F_{(2,35)}=3.156, p=0.057\right)$ showing that $24 \mathrm{~h}$ intake for KO INT mice tended to be lower, although this was not statistically significant. On non-access days, there was again a main effect of access $\left(F_{(2,35)}=10.069, p<0.05\right)$. Daily groups consumed a greater number of kcal $(16.3 \pm .6)$ on these days than both controls $(14.1 \pm .6)$ and INT mice $(12.8 \pm .5)$.

\section{Overall diet composition (Figure 2I).}

The percentage of kilocalories consumed from HFD across the experiment differed between access conditions $\left(F_{(1,26)}=106.463, p<0.05\right)$ and between genotypes $\left(F_{(1,26)}=4.654, p<0.05\right)$. DAILY mice consumed a greater percentage of their total calories from HFD than did the INT mice (Daily: $46.7 \% \pm 2.2 \%$ and INT: $15.7 \% \pm 2.1 \%$, $p<0.05)$ independent of genotype. In addition, the KO mice consumed a smaller percentage of calories from HFD, regardless of access condition (KO: $28.1 \%+/-2.2 \% \mathrm{vs}$. 
WT: $34.5 \%+/-2.1 \%, p<0.05)$. No significant interaction was detected $\left(F_{(1,26)}=.158, p>\right.$ $0.05)$. With regards to chow intake, the main effects for access condition $\left(F_{(1,26)}=\right.$ $106.463, p<0.05)$ and genotype $\left(F_{(1,26)}=4.654, p<0.05\right)$ were once again significant, but with the opposite trend, as can be seen in Figure 2I. Mice in the DAILY groups consumed less chow than those in the INT groups (Daily: $53.3 \% \pm 3.0 \%$ vs. INT: $84.3 \%$ $\pm 2.1 \%, \mathrm{p}<0.05$ ), and $\mathrm{KO}$ mice consumed a greater percentage of chow, independent of access condition (KO: $72.2 \% \pm 2.2 \%$ vs. WT: $65.5 \% \pm 2.1 \% \mathrm{p}<0.05$ ). GHSR KO mice when placed under either a limited or limited + intermittent access to a HFD maintain a lower intake of HFD than do their WT littermates over the course of the experiment.

\section{Body Weight (Figure 2J).}

As determined by two-way ANOVA (genotype $\mathrm{x}$ access) on body weight at completion of the experiment, there was a significant main effect of genotype $\left(F_{(1,35)}=\right.$ $5.048, p<0.05)$, such that KO mice weighed less than WT mice $(30.7 \pm 1.3 \mathrm{~g}$ and $34.8 \pm$ $1.3 \mathrm{~g}$, respectively). There was no main effect of access $\left(F_{(2,35)}=.313, p>0.05\right)$ and no interaction between genotype and access $\left(F_{(2,35)}=.102, p>0.05\right)$. 


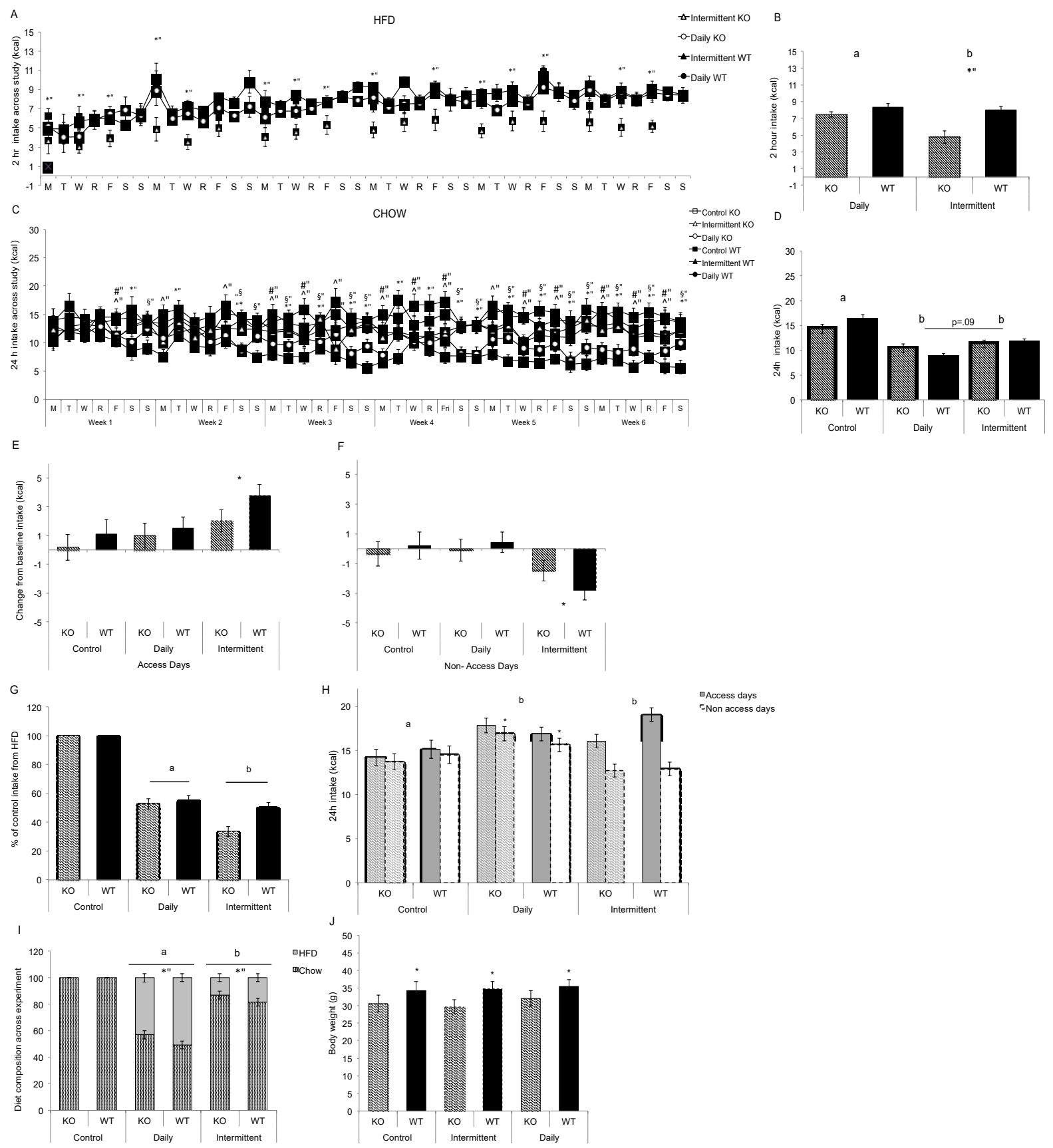

Figure 2 A-J. Feeding patterns, diet selection and body weight in C57-GHSR KO and WT mice. Caloric intake from HFD (2h) across the experiment (A) and overall average HFD intake on access days (B; a,b: $\mathrm{p}<0.05$; denotes access group differences; *, $\mathrm{p}<0.05$, denotes differences between $\mathrm{KO}$ and WT mice within the INT group). Mice receiving DAILY access to HFD consumed modestly more HFD than those in the INT access group. KO mice in the INT group did not eat as much HFD as INT WT mice. Patterns of caloric intake from chow across the experiment $(\mathrm{C}$; On access days: * denotes controls $>$ daily mice, $\S$ denotes that INT $>$ DAILY mice. On non-access days: \# denotes that INT mice $>$ DAILY mice, ${ }^{\wedge}$ denotes that DAILY $>$ controls; all symbols reflect 
$\mathrm{p}<0.05)$. Overall average chow intake on access days (D). Control mice ate the greatest amount of chow, followed by the INT and DAILY mice $(\mathrm{a}, \mathrm{b}, \mathrm{p}<0.05)$. Change from baseline intake on access days (E) and non-access days (F). INT access to HFD resulted in an elevated caloric intake on access days, and a reduction on non-access days, relative to baseline intake (main effect of access, ${ }^{*} \mathrm{p}<0.05$ ). Percent of average daily control group caloric intake consumed during 2 hour HFD diet exposure (G). DAILY groups consumed more than INT groups (main effect of access, ${ }^{*} \mathrm{p}<0.05$ ) and KO mice consumed less than WT mice (main effect of genotype, ${ }^{*} \mathrm{p}<0.05$ ), however there was a trend towards KO INT mice consuming less than WT mice (marginal interaction: $p=0.054) .24 \mathrm{~h}$ intake on access and non-access days $(\mathrm{H})$, showing that both INT and DAILY HFD access induced increases in total daily intake on access days $(a, b, p<0.05)$ and on non-access days DAILY mice ate the most, as they received HFD on these days. Diet composition across experiment (I). DAILY mice ate a greater percentage of their cumulative experimental calories from HFD relative to the INT mice (main effect of access condition, a,b: $\mathrm{p}<0.05)$. GHSR KO mice consumed a lower percentage of their kcal from HFD regardless of access condition (main effect of genotype, $\mathrm{p}<0.05$ ). Body weight at end of the experiment (I). A main effect of genotype revealed that GHSR KO mice were lighter than WT mice $(* p<0.05)$, but there was no main effect of access and no access by genotype interaction.

\section{C-fos IR in nucleus accumbens (Figure 3).}

One hour subsequent to their last receipt of HFD, mice were sacrificed and C-fos

IR was examined. The data in Figure 3 are expressed as the mean percentage of c-Fos

positive cells found in control mice. Two-way ANOVA revealed that there were no main effects of access $\left(F_{(1,24)}=0.060, p>0.05\right)$ or genotype $\left(F_{(1,24)}=1.31, p>0.05\right)$, and no interaction $\left(F_{(1,24)}=0.231, p>0.05\right)$ in expression in the NAcc core region, with all mice showing similar levels of C-fos expression following their final receipt of HFD.

Alternatively, we found that expression differed between WT and KO in the shell region of the NAcc (main effect of genotype: $F_{(1,33)}=8.003, p<0.05$ ), with KO mice showing a comparable amount of expression to controls, but with WT mice showing nearly a 3-fold increase in $\mathrm{C}$-fos expression relative to control mice. Table 1 depicts c-Fos counts expressed as percent of controls in both the NAcc core and shell, as well as the lateral hypothalamic area (LHA) and the ventral tegmental area (VTA). No main effect of access $\left(F_{(2,23)}=.479, p>0.05\right)$, genotype $(F(1,23)=.002, p>0.05)$, nor an interaction $\left(F_{(2,23)=} .002, p\right.$ 
$>0.05$ ) were found for expression in the LHA nor in the VTA (access: $F_{(2,23)=1.493, p>}$ 0.05; genotype: $\left.F_{(2,23)}=2.646, p>0.05\right)$; interaction: $\left.F_{(2,23)}=1.493, p>0.05\right)$, see Table 1 .

A

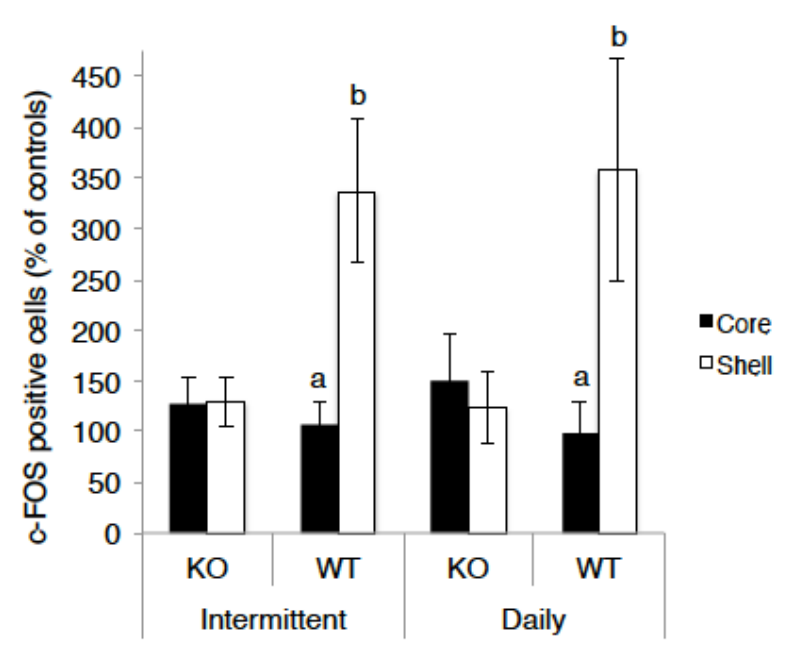

B

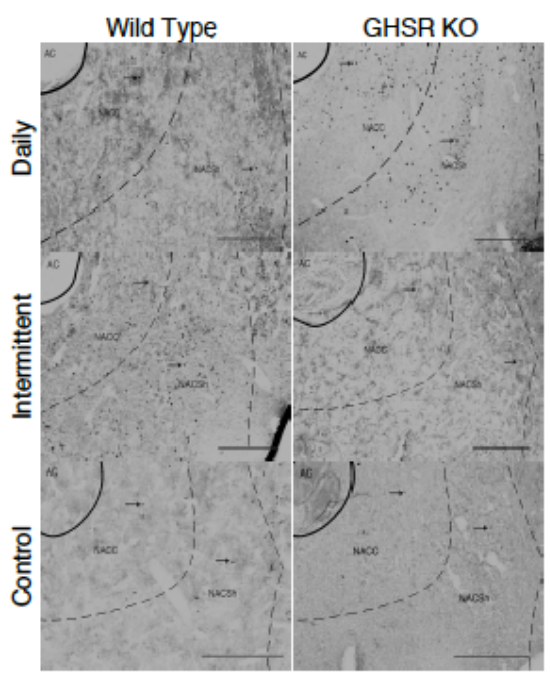

Figure 3. C-fos immunohistochemistry. A) Number of C-fos positive nuclei expressed as a percentage of control mice in the NAcc shell and core regions. WT mice that received the HFD on either a DAILY or INT basis showed elevated c-FOS IR in the shell region, relative to the $\mathrm{KO}$ mice on both access schedules $(\mathrm{a}, \mathrm{b}, \mathrm{p}<0.05)$. No significant differences in C-fos IR were found in the core region between groups. B) Representative photomicrographs of the shell region to visualize group differences (10X objective magnification; Bregma: $+1.54 \rightarrow+1.10$; Scale bar $=250 \mu \mathrm{m}$ ).

\section{Discussion.}

In the current experiments we examined the feeding patterns of mice subjected to a nutritionally complete HFD under limited access conditions. The aim of Experiment 2.1 was to attempt to replicate a rat model of binge eating in mice, originally developed by placing rats on a schedule of limited and sporadic access to fat (Corwin et al., 1998; Davis et al., 2007). Rats placed on this time-restricted (2 hours/day) and sporadic (3x/week) access to either vegetable shortening (Corwin et al., 1998), or a nutritionally complete high fat diet (Davis et al., 2007), display a binge/compensate pattern of feeding, typically evident by the second week of scheduled access. As this particular model has 
not yet been validated in mice, we sought to determine if mice subjected to a similar schedule of HFD access would exhibit a binge-like pattern of eating similar to that seen in rats. Furthermore, we were interested in determining whether a lack of functional ghrelin signaling in GHSR KO mice would hinder the development of binge eating in this model and examined post-HFD C-fos activity in the NAcc, a key target of VTA DA neurons that play a substantial role in modulating both appetitive and consummatory responses to foods with high palatability (Castro and Berridge, 2014).

In Experiment 2.1, we found that male CD-1 mice exposed to an INT access schedule demonstrated a binge/compensate pattern similar to that seen in prior studies conducted in rats (Corwin et al., 1998, Davis et al., 2007). However, mice with INT access did not eat an amount of HFD that was consistently in excess of those mice on the DAILY access schedule, although they did binge, consuming $85 \%$ of their average baseline caloric intake during the 2-hour HFD access period, meeting criteria for a binge episode (one example: consuming $>25 \%$ of average daily intake in a $1 \mathrm{~h}$ period; Halpern et al., 2013). Mice with DAILY access to the HFD consumed $71 \%$ of their average baseline calories during the HFD access period, though differences between access groups were not statistically significant. Therefore, mice exposed to HFD for $2 \mathrm{~h}$, either on a DAILY or INT access schedule, binged on HFD when given access to it. These results do not meet the operationalization of binge eating outlined by Corwin et al. (2011), as the intake of the palatable food source in the INT access group was not consistently in excess of the DAILY group, nor did it escalate substantially over time. However, the number of kcals consumed was similar to the size of binges in mice given 2h exposure to an HFD in the middle of the dark cycle, although these mice were 
exposed daily (i.e. predictably; Bake et al., 2013). The elevated total 24-hour caloric intake seen in INT mice on access days in our study can be attributed to the fact that the INT access mice continued to eat a similar amount of chow as they did during baseline, while bingeing on the HFD during the access period. The binge-compensation pattern of feeding was not only evident when comparing across access conditions but was also significantly different from the average baseline intake for each of the groups (see Figure $1 \mathrm{E}$ and F). Further, as seen in previous studies (e.g. Corwin et al., 1998), the number of kcals consumed from HFD over the course of the experiment was highest in mice that received HFD every day.

While not fully in line with previous rat studies demonstrating binge-like intake of exclusively high-fat diet, our findings from experiment 2.1 suggest that mice exposed to the HFD under the INT schedule do not compensate by decreasing their intake of chow during the dark period. This phenomenon has been reported in alternative animal models of overeating, in history of dieting and stress models (Hagan et al., 2003), as well as in studies examining cue-potentiated feeding on chow (Boggiano et al., 2009). In addition, intake of a less palatable food increased when rats were placed back into the context in which they previously received a palatable food (Boggiano et al., 2009). Allowing the animal to see and smell a palatable food but not allowing them to eat it, (as in the INT groups) can also induce an above average increase in corticosterone (CORT), a response that appears to be exaggerated in mice that tend to binge eat (Cifani et al., 2009). The removal of HFD prior to the natural feeding cycle may also serve as a stressor, thereby inducing increased consumption of the food that remains available. 
In Experiment 2.2, we used the limited access model on mice lacking GHSR in order to determine the role of ghrelin receptors in mediating binges by comparing them to their WT littermates. Mice in the DAILY access groups (both WT and KO) consumed modestly more HFD intake during the $2 \mathrm{~h}$ access compared to INT groups, demonstrating that both GHSR KO and WT mice increase their consumption of a HFD under this feeding paradigm. GHSR KO mice, however, consumed less of the HFD than their WT littermates when exposed to the INT HFD schedule. These results point to a role of GHSR in mediating the overconsumption of calories seen under the INT HFD schedule.

Reduced caloric intake may involve a number of mechanisms that include an increase in the ability of satiety hormones to stop HFD feeding bouts in GHSR KO mice (Zigman et al., 2005). Alternatively, the lack of GHSR signaling may prevent ghrelin from interacting directly with dopaminergic neurons via GHSR in the midbrain and decrease overall motivation to eat the HFD on an INT HFD feeding schedule. The latter hypothesis is supported by our data examining cFOS IR in the NAc. GHSR KO mice post-HFD showed lower levels of cFos activation in the NAc, particularly in the shell region following consumption of HFD. Interestingly, although WT mice DAILY and INT groups did not differ in HFD intake, they did show elevated levels of cFos IR in this same region relative to control and GHSR KO mice. Therefore, our results support the notion that the NAcc is recruited following acute consumption of HFD in naïve mice (Valdivia et al., 2014) and is an important component of the circuitry underlying foodseeking behaviors (Kelley, 2004; Di Chiara and Bassareo, 2007). Additionally, the importance of the glutamatergic system within the NAcc shell region, but not the core, in maintaining binge eating was also recently demonstrated in rats (Smith et al., 2014), 
suggesting a potentially greater role for the shell in regulating excessive or binge-like consumption of palatable foods.

Direct action of ghrelin on GHSR in the VTA can excite DA neurons, as shown by rapid induction of increased excitatory and reduced inhibitory inputs onto tyrosinehydroxylase $(\mathrm{TH})$ expressing neurons upon intra-VTA infusion. Intra-VTA ghrelin also increased TH neurons firing rates, as well as increased DA turnover in the NAc (Abizaid et al., 2006). Interestingly, we did not find exaggerated c-Fos expression in the VTA or in the lateral hypothalamic area (LHA), nor differences between groups. Both of these regions have been shown to be activated in response to HFD and important for expression of food reward behaviors (e.g. Perello et al., 2010; Valdivia et al., 2014; Valdivia et al., 2015). It is possible that if we had looked at anticipatory rather than post-ingestive C-fos profiles, we may have seen differences between groups, as mice that lack GHSR show an attenuated C-fos profile in the VTA, as well as a reduced proportion of orexin neuron activation in the LHA (Lamont et al., 2012). It is possible that ghrelin's direct action at the level of the VTA could become less prominent as the length of exposure to the palatable food increases, and that other ligand-independent interactions with alternate GPCRs in the NAc, such as the D1R, could facilitate the intake of HFD (for review see: Wellman and Abizaid, 2015). Lack of GHSR in this case would alter DA receptor activity in the NAc, without altering activity in the VTA or the LHA.

Activation of the NAcc upon chronic exposure to a palatable food may reflect how palatable the mice find the food, but may no longer require recruitment of VTA-DA after chronic repeated exposure. It has also been shown that direct action of ghrelin on the NAc is sufficient to induce a feeding response (Naleid et al., 2005) as well as to enhance 
locomotor responses to cocaine in rats (Jang et al., 2013), and therefore a lack of GHSR in NAc could suppress overeating of HFD that would typically be induced by ghrelin stimulation in this region. The higher levels of c-Fos seen in the shell region under both DAILY and INT access conditions only in WT mice, indicate that this region is activated to a greater extent following consumption of a HFD when animals have functional GHSRs. Although the precise relationship between c-Fos expression and transcription and/or depolarization is unknown (Hoffman, Smith and Verbalis, 1993), it is still a powerful method of detecting activation in response to a given stimuli, albeit with poor time-course precision. Determining the phenotype of the neurons exhibiting c-Fos IR in this study would add to our knowledge about which subpopulation of neurons in the NAcc are being activated in the context of this model.

With regards to change from baseline $24 \mathrm{~h}$ caloric intake, mice with intermittent access to HFD increased intake on access days, and reduced intake on non-access days, consistent with the binge-compensate pattern of intake seen in our first experiment, as well as in previous reports employing this model in rats. Interestingly, there was no effect of genotype on this measure, suggesting that $\mathrm{KO}$ mice are able to develop the same patterns of intake when placed under these access conditions, but do not binge on HFD to the same extent during HFD access periods.

Diet composition in experiment 2.2 was similar to composition data found in experiment 2.1, with mice receiving DAILY access to HFD deriving a greater percentage of their kilocalories from HFD than did the INT groups. Additionally, we found that KO mice, whether receiving DAILY or INT access did not consume as great a percentage of kcals from HFD as WT mice. Therefore, over a 6-week period, GHSR KO mice show 
reduced consumption of HFD when access to it is restricted, whether they receive access daily or only 3 days per week. Rodents lacking ghrelin signaling or receiving a GHSR antagonist have a reduced ability to form a conditioned place preference to HFD (Perello et al., 2010; Chuang et al., 2011), a reduction in cue-potentiated feeding (Walker, Ibia and Zigman, 2012) and reduced consumption of a high-fat "dessert" following a satiating meal (St.Onge and Abizaid, 2012). Therefore, it is clear that intact GHSR plays a role in increasing the consumption of a HFD, although we cannot comment here on a potential decrease in palatability of the HFD to mice lacking GHSR as we did not directly measure "liking" responses (Shin et al., 2010; Overduin et al., 2012). The regions within which GHSR activation may be important for such behaviors are currently being explored.

The lack of two-hour consumption differences between access groups may suggest that the diet chosen, as it was a nutritionally complete HFD, may have led the groups to both consume such large amounts that differences between access groups could not be detected. The possibility exists that the HFD was too palatable and therefore drove excessive consumption in both access conditions. However, as previous experiments have demonstrated that a nutritionally complete HFD can induce a binge compensate pattern of intake in intermittent access mice comparable to vegetable shortening (Davis et a., 2007), we chose to use this diet in our current experiments. Further, there is often overlap in fat intakes between INT and DAILY access groups, and in some cases, up to half of the animals from each group can be matched on short-term (1h) fat consumption (Wojnicki, Babbs and Corwin, 2010). This can occur throughout the course of the experiment and highlights the possibility of high variability within access groups and could help to explain some of our discrepant findings in mice. Additionally, not all humans who are 
exposed to highly palatable fatty foods under an intermittent schedule, whether selfimposed or not, exhibit binge-like eating. Exploring the underlying mechanisms in those animals and humans prone to binge eating and those not prone, as well as the role of ghrelin in such models, may serve to highlight some of the underlying mechanistic differences between these groups.

In a recent study conducted by Valdivia et al. (2015), it was shown that $2 \mathrm{~h}$ HFD consumption over a 4-day period was sufficient to cause an escalation of intake and that a lack of GHSR eliminated this escalation across days. The HFD access group in this study was akin to our DAILY mice, in that they received HFD every day at the same time for 4 days in a row. We did not find an escalation of intake in our experiment, and it may be that GHSR KO mice given longer-term predictable exposure to the HFD retain the ability to match the HFD intake of the WT mice after the first few days of intake. The unpredictability of the HFD access in the KO INT mice appears to contribute to the reduced binge-like consumption, potentially reflecting an inability of the GHSR KO mice to take advantage of limited availability of the palatable diet, as the WT mice do. It has been shown that mice administered a ghrelin antagonist during the acquisition phase of conditioned place preference for HFD failed to develop a preference, but that once acquired, the preference was not hindered by blockade of ghrelin on test day (Perello et al., 2010). Therefore, it is also possible that a lack of ghrelin signaling via GHSR during palatable food access that is more uncertain, GHSR KO mice lack the ability to orchestrate a full binge-like response. The anatomical and functional correlates of such behavior have yet to be explored in the context of this model. 
Overall, we have shown that the two strains exhibited both similarities and differences in behavior when subjected to INT and DAILY access to palatable food. All mice over-ate on access days and under-ate on non-access days, as a result of adding 2 hour access to palatable food to their ad lib chow dietary regimen, showing that exposure to palatable foods in the absence of energy requirements enhances daily caloric intake. However, caloric consumption above and beyond baseline daily intake in CD-1 mice appeared to be a combination of consistent chow intake despite limited access to HFD, whereas in the C57BL6/J mice, intake of chow was not elevated in HFD access conditions, and in fact was lowered in mice that received DAILY HFD. Strain differences can affect the feeding behavior and metabolic parameters of mice given a HFD continuously (Sims et al., 2013; Nishikawa et al., 2007), however, there are only a few recent studies that examine binge-like feeding in mice and they used C57BL6/J mice (Czyzyk et al., 2010; Cao et al., 2014) and some examined sucrose (Yasoshima and Shimura, 2015) or chow intake (Razzoli et al., 2015). This makes it difficult to compare our CD-1 findings with those of other studies. However, as the few murine binge-like feedings paradigms utilize C57BL6/J mice, and as many genetically engineered mouse models are backcrossed onto this strain, we have continued to use these mice in our studies.

Upon examination of the limited access model in GHSR KO and WT mice, there appears to be a role for GHSR signaling in the amount of HFD consumed under these conditions, as GHSR KO mice fail to consume as much HFD across the study when access is restricted, and these results are enhanced when the access is scheduled in a less predictable manner (i.e. MWF). The mechanisms by which the lack of intact GHSR 
lessens the magnitude of the "binge" under intermittent access schedules in KO mice are unknown. It has been hypothesized that the interaction of ghrelin with mesolimbic reward circuitry serves to amplify the pleasure derived from a number of reinforcers (Lockie et al., 2015), and the lack of ghrelin-dependent GHSR signaling to reduce responding for stimuli such as cocaine (Abizaid et al., 2011) or nicotine (Jerlhag and Engel, 2011) supports this hypothesis. We are currently investigating the role GHSR signaling in various brain regions that may play a role in the development of binge-like behavior.

\section{Conclusion}

Taken together, data from both experiments support the ability of intermittently scheduled access to palatable food in mice to induce a binge-compensate pattern of intake that emulate select components of binge eating in humans. Additionally, there appears to be a role for GHSR signaling in the amount of HFD consumed under these conditions. The reciprocal relationship between intermittent feeding schedules and the neuroendocrine regulation of feeding behavior, as well as its underlying mechanisms remain poorly studied (for review see Murphy and Mercer, 2014), and the precise role of the ghrelin system in this process remains to be determined. Additional insight into these processes may aid in the understanding of binge eating and how this pattern of disordered eating develops and persists.

\section{Acknowledgments}

This work was supported by an NSERC discovery grant (AA) and an NSERC Alexander Graham Bell PGS (SJK). 
Table 1. Percent of control c-Fos IR in select brain regions.

\begin{tabular}{|c|c|c|c|c|}
\hline \multirow[t]{2}{*}{ Region } & \multicolumn{2}{|c|}{ Daily } & \multicolumn{2}{|c|}{ Intermittent } \\
\hline & $\mathrm{KO}$ & WT & $\mathrm{KO}$ & WT \\
\hline NAc Shell & $123.2 \pm 34.9^{*}$ & $357.8 \pm 109.5$ & $129.5 \pm 25.3^{*}$ & $336.5 \pm 70.6$ \\
\hline NAc Core & $150.7 \pm 45.5$ & $99.1 \pm 29.6$ & $127.7 \pm 27.15$ & $106.6 \pm 22.6$ \\
\hline $\mathrm{LH}$ & $106.9 \pm 26.24$ & $81.36 \pm 29.4$ & $105.923 \pm 29.4$ & $134.99 \pm 26.24$ \\
\hline VTA & $107 \pm 41.8$ & $210.3 \pm 37.4$ & $52.26 \pm 48.2$ & $130.8 \pm 41.8$ \\
\hline
\end{tabular}

* indicates significant difference $(\mathrm{p}<0.05)$. 


\section{Chapter 4: Exploring the contribution of the GHSR in the ventral tegmental area (VTA) on binge-like consumption of a high-fat diet (Studies 2.3 and 2.4).}

\section{Introduction}

Excess caloric intake driven by ubiquitous food cues and easy access to energy dense foods has contributed to an increase in eating disorders and metabolic issues related to chronic overconsumption. Interest in the variables that drive overconsumption of energy dense foods, has piqued in recent years, due to the experiences of individuals that find themselves unable to curb their intake of energy dense foods, as well as from the concern of clinicians attempting to mitigate the health consequences of such eating habits. When overconsumption of such foods occurs over extended periods, psychological distress, obesity and associated impairments of metabolic function can result. In humans, each of these symptoms can manifest in the context of binge eating disorder (BED), wherein people consume an excessive amount of food in a short time frame (i.e. and episode) and often perceive a loss of control over their intake, continuing to eat despite a lack of energetic demand (APA, 2013).

Based on findings demonstrating enhanced intake of substances following restricted access to them (Wayner and Fraley, 1972; Pinel and Huang, 1976; Corrigal and Cohen, 1989), Corwin and colleagues (1998) developed a model of limited access to lard that is able to reliably induce binge-like consumption in rats. The feeding patterns generated using this model exhibit many similarities to individuals who suffer from BED. Male and female rats with intermittent access binge on the fat during access and escalate their consumption of the fat over time (Dimitrou, Rice, and Corwin, 2000; Corwin, 2004; Wojnicki, Babbs and Corwin, 2010). Interestingly, these studies do not demonstrate a substantial shift in body weight in rats that binge, which allows for investigation of 
feeding patterns in the absence of potentially confounding weight gain. Another strength of this model is the ability of the intermittent access schedule to induce voluntary bingeing without requiring a prior history of food restriction or stress, allowing for examination of vulnerabilities to the initial development of disordered eating.

Abnormalities in reward circuits within the central nervous system (CNS) are thought to partially underlie binge eating, however, most studies have been carried out in diet-induced obese (DIO) rodents or obese humans, despite the lifetime prevalence of obesity in those that in those with BED being only $36 \%$ (as indicated by BMI; Kessler et al., 2013). Still, many parallels can be drawn between eating patterns that occur in the context of BED and addiction-like phenotypes (Volkow et al., 2008; Michaelides et al., 2012; Johnson and Kenny, 2010). This raises the possibility that mesolimbic dopamine (DA) release, from DA cell bodies in the ventral tegmental area (VTA) into the ventral striatum (Wise and Rompre, 1989; Wise, 2006), is involved in the overconsumption of foods that are high in fat and/or sugar, in part, due to enhanced responsiveness to food related cues (Roitman et al., 2004; Day et al., 2007). These are also the types of food that are often consumed during binge episodes (Kales, 1990). Upon first consumption of a palatable food, the firing of DA neurons in the VTA increases, with a downstream elevation of DA release in the NAc (Norgren et al., 2006). Short term exposure (24h) to a sweetened high-fat food also rapidly enhances glutamatergic excitatory inputs onto VTA DA cells, enhancing motivation to enter an aversive compartment to seek palatable food and increasing consumption of such food (Liu et al., 2015), illustrating the neuroplastic changes that occur in VTA DA neurons as a result of palatable food exposure. 
As mesolimbic circuitry is also sensitive to circulating feeding peptides (for review, see Liu and Borgland, 2015), it is possible that one or more of these peptides influence the consumption of palatable foods through their action on VTA DA neurons. The orexigenic peptide ghrelin is produced primarily in the stomach (Kojima et al., 1999; Date et al., 2000), but can act centrally to increase food consumption (Abizaid et al., 2006), reduce energy expenditure and promote fat storage (Tschop et al., 2000). In terms of hedonic feeding (i.e. feeding driven by the palatability of the food being offered rather than by a physiological energy deficit), ghrelin and its receptor, the growth hormone secretagogue receptor (GHSR), appear to play an important modulatory role. Microinfusions of ghrelin into to the VTA facilitate rapid synaptic plasticity to enhance VTA DA output to the NAc (Abizaid et al, 2006), enhance the motivation to obtain palatable foods (King et al., 2011; Skibicka et al., 2011; Skibicka et al., 2012), and increase the preference for and consumption of palatable foods and liquids high in fat and/or sucrose (Shimbara et al., 2004; Disse et al., 2010; Egecioglu et al., 2010; Perello et al., 2010). Conversely, rodents lacking GHSR show deficits in food reward behaviours (Chuang et al., 2011; Perello et al., 2011; King et al., 2016; MacKay et al., 2016). GHSRs are found in the VTA of rats and mice (Guan et al., 1997; Zigman et al., 2006) and approximately $60 \%$ colocalize with cells expressing tyrosine hydroxylase (TH; Abizaid et al., 2006). Therefore, interactions between the GHSR and DA may influence the consumption of palatable food and play a role in the development of binge-eating.

Evidence for the involvement of ghrelin signaling via the GHSR in the context of high-fat diet (HFD) consumption and binge eating has been demonstrated in our previous work. In our recent study, mice that lack GHSRs demonstrate attenuated binge intake of 
HFD when it is presented on an intermittent access schedule (INT; MWF) for $2 \mathrm{~h}$. GHSR KO mice also show reduced cFos immunoreactivity (IR) in the NAc shell (NAcSh) following their last binge. Valdivia et al. (2015) demonstrated that acute exposure (2h) to a pellet of HFD in naïve mice activated the mesolimbic circuit, and that LH orexin signaling was required for this response (Valdivia et al., 2015). Their follow-up study examined patterns of food intake and neural activation as indicated by c-Fos, over a 4day period in mice with genetic deletion of the GHSR. They found that GHSR deficient mice did not escalate their HFD intake to the same extent as the WT mice and had less cFos activation of the VTA following HFD consumption (Valdivia et al., 2015). Wei et al. (2015) demonstrated that ghrelin administration directly into the VTA was able to enhance consumption of HFD when rats were either hungry or satiated, and that this effect could be blocked by administration of the GHSR antagonist D-Lys-3-GHRP-6. Bingeing on fat during adolescence also upregulated GHSR mRNA in the VTA and enhanced the reinforcing effects of cocaine (Blanco-Gandia et al., 2016). Together, these results suggest that GHSR signaling (whether ligand -dependent or independent has yet to be determined) could underlie the robust binge-like intake of HFD when presented in a time restricted fashion. Interestingly, the effects of a lack of GHSR signaling on the binge-like intake of HFD in our previous study appears most pronounced under an intermittent (INT) schedule of limited access to HFD. The implications of such findings in terms of a role for the GHSR on binge intake of high-fat have not yet been elucidated, however, we hypothesized that the VTA might be an important neural substrate for the role of ghrelin and the GHSR in high-fat bingeing. Therefore, in this set of experiments, we sought to determine whether GHSR signaling specifically in the VTA is important for 
the full expression of this behaviour. Recent studies from our lab have also demonstrated the importance of intact ghrelin signaling in palatable food intake in rodents. Rats with a truncated ghrelin receptor (FHH-GHSR ${ }^{\mathrm{m} / \mathrm{Mcw}}$ ) show reduced intake of a palatable and energy-dense "dessert" when satiated, relative to WT rats (MacKay et al., 2016). GHSR signaling is also important in developing a conditioned place preference (CPP) for HFD in food restricted mice (Perello et al., 2010). These data provide a strong rationale for our hypothesis that GHSR signaling in this region is important for binge-like consumption of palatable food, even in the absence of an instrumental requirement to obtain the food.

In addition to discerning whether GHSR in the VTA is important for the generation of binge-like intake of HFD, we were also interested in determining whether antagonism of the GHSR in this region might provide therapeutic potential for binge-like eating once it has already been established. While data utilizing rodent models of binge eating may not be able to recapitulate the full range of physiological and psychological factors present in humans with BED (see brief review: Perello et al., 2014), findings from these studies are still important for understanding the etiology and maintenance of binge eating behavior. Many of the findings pertaining to the ghrelin system, particularly in relation to enhancing overall food reward, including appetite, feeding, and enhanced palatability of food extend to human samples (Wren et al., 2001; Schmid et al., 2005; Druce et al., 2005; Malik et al., 2008). While it would be difficult to administer a GHSR antagonist to the VTA directly in humans to reduce binge eating, it is still useful to know whether GHSR activity in this region is responsible for initiating and sustaining bingelike consumption of diets high in fat. This would be particularly interesting to determine 
under conditions where access is limited and/or intermittent, considering the prevalence of voluntary dieting in populations of individuals who binge eat and/or are overweight. In the first study, we use an adeno-associated viral (AAV) vector delivering crerecombinase (CRE) to reinstate GHSR mRNA transcription specifically in neurons of the VTA in adult GHSR null mice (Zigman et al., 2005, see Appendix A). Administering the virus to any region of the brain that expresses the GHSR results in excision of a transcriptional blocking cassette (TBC) that is floxed by two loxP sites, upstream of the promoter region of the GHSR. If GHSR signaling in the VTA is required for the full development of binge-like intake of HFD, we hypothesize that GHSR null mice will show attenuated binge-like intake with exposure to the limited access model, and that reinstatement of GHSR in the VTA will increase bingeing to approximate WT mice.

In the second study, we aim to determine whether blocking GHSR signaling in the VTA in mice is effective at reducing bingeing once the behaviour has already been established. We hypothesized that administration of a GHSR antagonist chronically into the VTA would attenuate binge-like feeding on a high-fat diet under the limited access model. These data will contribute to the emerging body of literature examining the importance of mesolimbic circuity in the context of bingeing on palatable food and determine whether GHSR activity in the VTA is an important neural substrate contributing to this phenotype. 


\section{Experiment 2.3. Adeno-associated viral rescue of the GHSR and its influence on high-fat bingeing under the limited access model.}

\section{Materials and Methods.}

Animals.

The transgenic mice used for this experiment were originally obtained from the University of Texas Southwestern Medical Centre (Zigman et al., 2005), and an in-house colony was maintained in our facility. Genotype was determined using PCR performed on ear tissue punches obtained at the time of weaning. See transgenic section in Chapter 2, Study 1.3.1 for more detail on the nature of the mutation in these mice.

Adult male and female transgenic GHSR null and WT mice were bred in house for use in this study. Mice were housed individually at the start of the experiment in a temperature and humidity-controlled vivarium with a 12:12 light/dark schedule (Lights on: 07:00 to 19:00). All mice had ad libitum access to water and nutritionally complete standard laboratory chow (Teklad Global 14\% Protein Rodent Maintenance Diet 2014, Harlan Laboratories, Madison, WI, USA; percent of kilocalories derived from fat: 13, protein: 20 , carbohydrates: $67 ; 2.9 \mathrm{kcal} / \mathrm{g}$ ) for the duration of the study. Body weight and food intake were measured daily, with the first 7 days serving as a baseline. At the end of the baseline period, mice were given access to a nutritionally complete HFD overnight (Open Source Diets, D12492; Research Diets, Inc., New Brunswick, NJ; percent of kilocalories from fat: 60 , protein: 20 , carbohydrates: $20 ; 5.24 \mathrm{kcal} / \mathrm{g})$, to prevent neophobia prior to the start of the limited access model. Mice were initially divided into 6 groups: GHSR null CONTROL $(n=16)$; GHSR null DAILY $(n=16)$; GHSR null INT $(n=16)$; WT CONTROL $(n=16)$; WT DAILY $(n=16)$; WT INT $(n=16)$. After 6 weeks on the limited access model, groups were split, with half of the mice in each group receiving the control GFP AAV and the other half receiving the Cre-GFP AAV into the VTA. 
Following surgery, mice were kept on the limited access model for an additional 3 weeks and sacrificed upon completion of the final binge.

\section{Limited access model.}

Mice were given ad libitum access to standard chow over the course of the experiment. Food intake and body weight were measured daily. For six weeks, mice were given either: daily access to the HFD (DAILY) for 2 hours beginning at 15:30; given access to the diet only 3 times per week (INT; MWF) at the same time; or never given access to the HFD (Control). HFD was placed in small glass flat-bottomed bowls in the home cage of the mice on the days they were scheduled to receive HFD. The amount of HFD consumed during the 2-h period was recorded. At the time of HFD removal, the bottom of the cage was examined to ensure that no spillage was left unrecorded

Following six weeks on the limited access model, mice received surgical delivery of the assigned AAV and remained on the limited access model for an additional 3 weeks to determine the effects of reinstatement of GHSR in the VTA on high-fat binge intake. Following the last binge, mice were decapitated and their brains were flash frozen and stored at $-80^{\circ} \mathrm{C}$ until further processing.

\section{Surgical delivery of adeno-associated viral vectors.}

Delivering cre-recombinase via adeno-associated viral vectors (AAVs) to the GHSR null mice allows for rescue of the GHSR in adult mice with spatiotemporal precision. Surgery was conducted using a gaseous anaesthetic (Isofluorane/Oxygen mixture; $2-5 \%$ delivered in $1 \mathrm{l} / \mathrm{min} \mathrm{O}_{2}$ ). Mice were anaesthetized and placed into a stereotaxic apparatus to allow for accurate localization of the target region (VTA). An incision was made in the skin above the skull, the skin was retracted and bregma was located. Following demarcation of the target infusion site (VTA co-ordinates: AP: -2.95; 
ML: +/-0.7; DV: -4.55), a small hole was drilled for insertion of the injector and delivery of the virus (bilateral infusions: rate: $75 \mathrm{nl} / \mathrm{min} / \mathrm{side}$; volume: $0.5 \mu 1 / \mathrm{side}$ ). Infusions were delivered using a 33-gauge injector connected via PE 20 tubing to a $10 \mu \mathrm{l}$ Hamilton airtight syringe attached to a GenieTouch ${ }^{\mathrm{TM}}$ dual-syringe pump (Kent Scientific Corporation). Half of the mice in each group were infused with either the GFP-only AAV (Control: AAV9.hSyn.eGFP.WPRE.bGH) or the CRE-GFP-AAV (Cre:

AAV9.hSyn.HI.eGFP-Cre.WPRE.SV40; Penn Vector Core, Gene Therapy Program, UPENN). The skin was then sutured, the incision site disinfected, and topical analgesic $\left(\right.$ Emla $\left.^{\mathrm{TM}}\right)$ and antibiotic (Polysporin $\left.{ }^{\mathrm{TM}}\right)$ were applied. Mice also received an injection of analgesic (Metacam) prior to start of surgery and for 3 days post-operatively, to ensure adequate pain relief. Mice were maintained on their corresponding HFD access schedule for the duration of the study, including the day that they received surgery.

Verification of GHSR mRNA expression in the VTA.

$R N A$ extraction and $c D N A$ synthesis.

Three weeks following stereotaxic infusion of the AAV into the VTA, mice were decapitated, brains were extracted and flash frozen in ice-cold 100\% ethanol (EtoH) and stored at $-80^{\circ} \mathrm{C}$ for later processing. Total RNA from bilateral edinger westphal nuclei (EW) and VTA brain punches were isolated from homogenized brain punches using TRIzol (Life Technologies) and precipitated using linear acrylamide. The purity and concentration of extracted RNA was quantified by the ratio of absorbance at $260 \mathrm{~nm}$ over 280nm with a ThermoScientific Nanodrop 100 spectrophotometer (ThermoScientific, Rockford, Illinois). RNA quality was determined by gel electrophoresis. cDNA synthesis was conducted using the iScript cDNA synthesis kit (BioRad Laboratories Inc., Hercules, California). 
Real-time quantitative polymerase chain reaction (RT-qPCR).

RT q-PCR was conducted on all cDNA samples to determine relative expression of target genes [ $\beta$-actin (reference gene) and GHSR (gene of interest)], using the $\Delta \Delta \mathrm{Ct}$ method. $5 \mu 1$ of cDNA sample was added to each well of a 96-well PCR plate, followed by $2 \mu 1$ of working concentration of primer solution, $3 \mu 1$ of DEPC water, and $10 \mu 1$ of SsoAdvanced ${ }^{\mathrm{TM}}$ SYBR ${ }^{\circledR}$ Green Super Mix with Fluorescein (Bio-Rad Laboratories Inc, Hercules, California). Samples were run in duplicate alongside non-template controls (NTCs) and positive controls. The plates were run on a CFX Real-time PCR detection system (Bio-Rad) and data will be analyzed using CFX Manager 3.0 software. The protocol consisted of 30 seconds at $95^{\circ} \mathrm{C}$, followed by 45 cycles of the following: 10 seconds at $95^{\circ} \mathrm{C}$ denaturing, 30 seconds at $55^{\circ} \mathrm{C}$ for annealing, and 20 seconds at $72^{\circ} \mathrm{C}$ for extension. The final steps include 1 minute at $95^{\circ} \mathrm{C}$ and 1 minute at $55^{\circ} \mathrm{C}$. Primers were initially tested for amplification efficiency using the standard curve method and yielded over 90\% efficiency. Primers were purchased from Eurofins Genomics (Huntsville, Alabama), and were identical to the primers used in Chapter 2.

\section{Statistical analyses.}

Data were analyzed using mixed ANOVAs using time as the within-subjects factor (3 time points: 'Week 1,' the first week of access condition; 'Week 6', the last week of access prior to surgery, and 'Week 9', the last two weeks of viral infection), and genotype (WT/GHSR null), sex (M/F), access schedule (control, DAILY or INT) and virus $(\mathrm{GFP} / \mathrm{CRE})(2 \times 2 \times 3 \times 2)$ as the between group factors. Follow up multiple comparisons using Fisher's LSD were performed. Three-way or two-way ANOVAs (genotype $\mathrm{x}$ access $\mathrm{x}$ virus) on overall average data were conducted when necessary, and Fisher's LSD post-hoc tests used to determine specific group differences. Data from this 
experiment are expressed as mean \pm standard error of the mean (SEM). All statistical analyses were performed with SPSS 20.0.0 for Macintosh (IBM Corp., Armok, NY) and statistical significance was set at $\alpha=0.05$.

Results.

Confirmation of GHSR rescue (qRT-PCR) (Figure 1).

GHSR was restored within the VTA to approximately $30 \%$ of the levels of WT

mice (see Figure 1A) whereas minimal GHSR restoration was observed in the control region (Figure 1B) and the level observed in the GFP mice was only $0.07 \%$ of controls. In spite of our ability to rescue this proportion of GHSRs, the overall analysis showed no indication that the rescue influenced any of our dependent variables, and thus genotype became the variable of greatest interest.

A

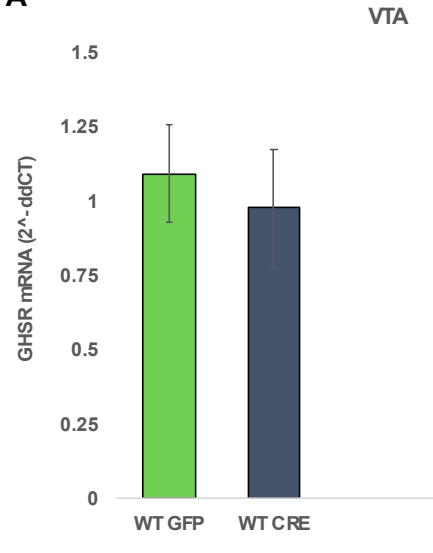

B
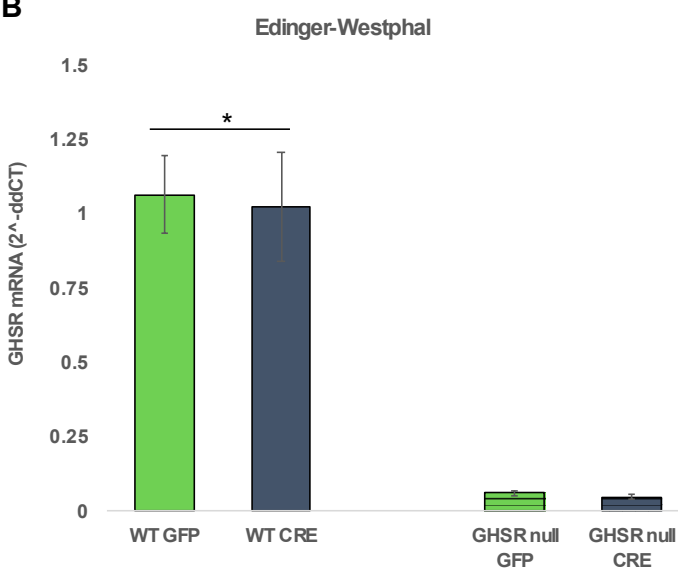

Figure 1. q-RT-PCR verification of GHSR rescue. GHSR were partially rescued in GHSR null mice following infusion of the AAV delivering CRE $\left({ }^{*} p<0.05\right)$.

Effect of genotype and access condition on binge behavior (Figure 2).

A recent paper suggested that a 2 -fold increase in caloric consumption, as compared to control mice during the same period, is sufficient to be considered a binge episode (Perello et al., 2014). Others have proposed an episode require $>25 \%$ of daily caloric intake in a 1-hour period (Halpern et al.,2013). Mice in our current studies 
consumed between $30-81 \%$ of their average daily intake from the HFD, showing that they all exhibited binge behaviour. As can be seen in Figure 2, this level was reached by mice in both the DAILY and INT access conditions in Week 1. Using percent of baseline daily caloric intake as a measure of binge size, mice in the DAILY group had larger binges than mice in the INT group (main effect of access: $F_{(1,49)}=4.630, p=0.036$ ) and female mice smaller binges than males, but only if they lacked the GHSR (genotype by sex interaction: $F_{(1,49)}, 6.071, p=0.012$; see Figure 2). All mice increased binge size over the course of the experiment (main effect of time: $F_{(2,98)}, 82.344, p=0.000$ ), suggesting that this was a diet that they preferred. There were no additional significant interactions.

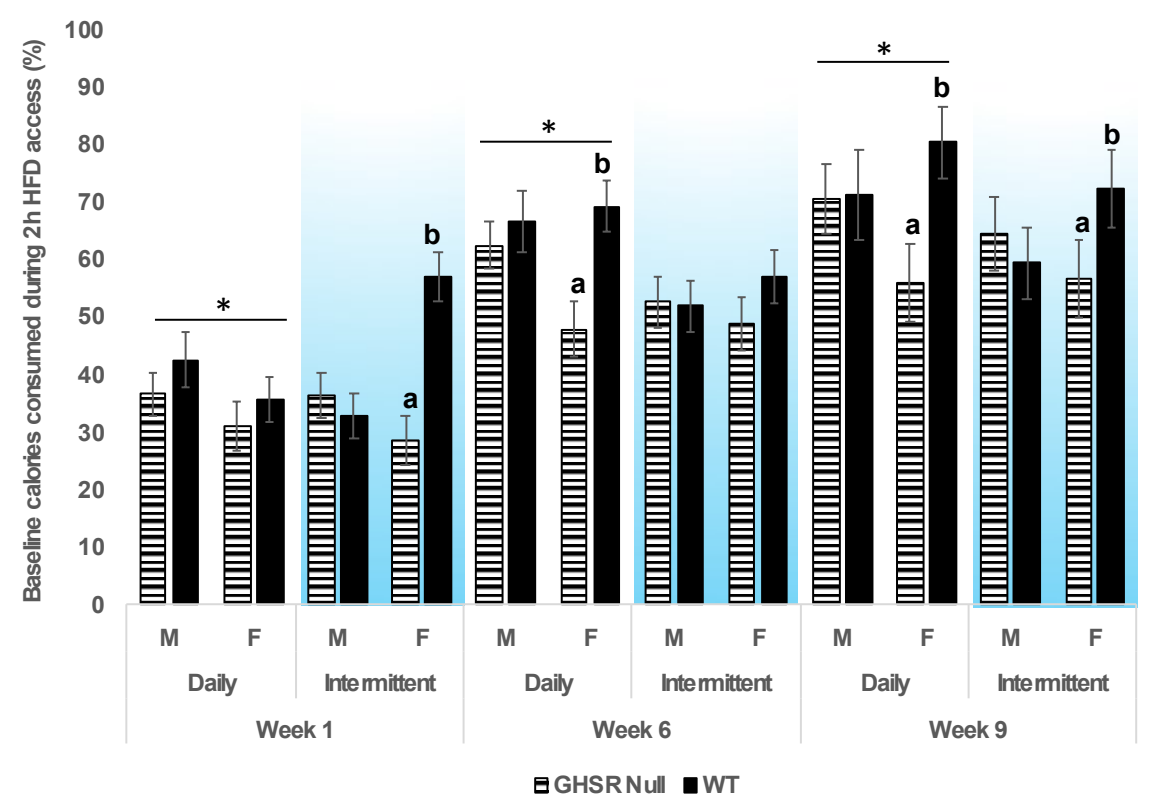

Figure 2. Binge Behaviour. Over time, mice increased binge size and mice in the DAILY group had larger binges than INT mice (main effect of access, *, $p<0.05$ ) whereas those of female mice were smaller, but only if they were GHSR null mice (genotype * sex interaction, a,b, $p<0.05$; blue background indicates INT access).

HFD intake during the $2 \mathrm{~h}$ access period (Figure 3 ).

In contrast to evaluating caloric intake from HFD as a function of baseline caloric intake, neither access condition nor genotype significantly affected the absolute number 
of calories eaten from HFD. Trends were observed, however, such that WT mice consumed more than GHSR null mice $\left(F_{(1,57)}, 3.147, p=0.070\right)$, and mice in the DAILY group consumed more calories during HFD access than those in the INT group $\left(F_{(1,57}\right.$, $3.714, p=0.059)$. The marginal genotype trend is consistent with the results of our previous experiment (King et al., 2016; Chapter 3), using a different GHSR KO mouse strain.

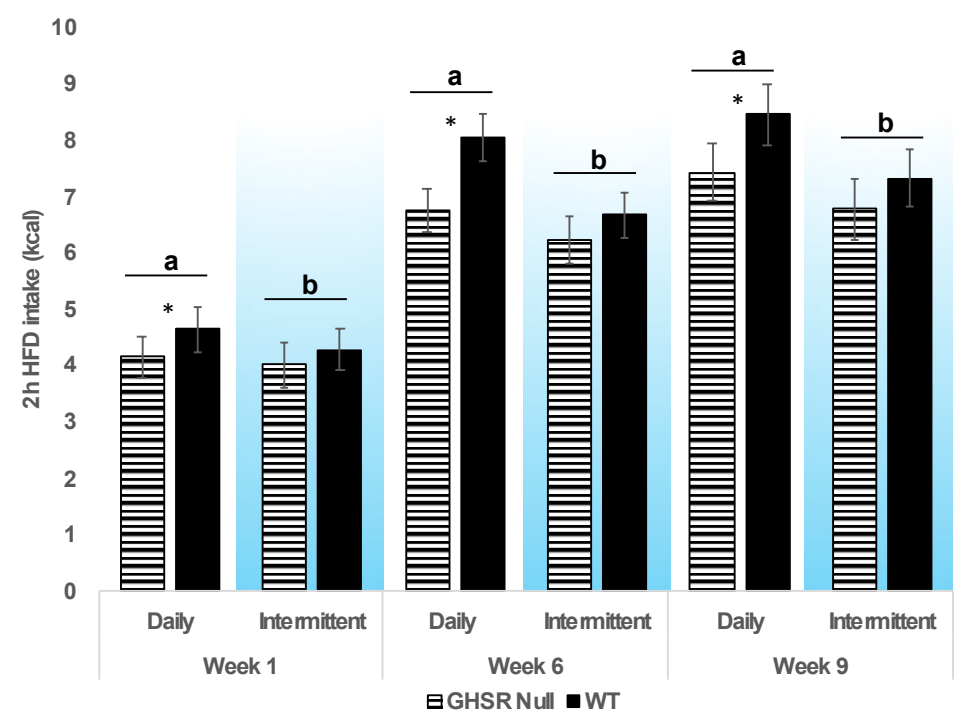

Figure 3. Average high-fat diet intake. Mice receiving access to a HFD DAILY, showed a trend towards consuming more fat than mice in INT groups (marginal effect of access; $a, b, p=0.059)$. WT mice also showed a trend towards consuming more HFD than GHSR null mice (marginal effect of genotype; $*, p=0.070$ ).

Effect of genotype and access condition on Chow intake (Figure 4A,B,C).

Chow intake on access days.

Overall, there was no effect of genotype on chow intake on access days but as expected, access condition did impact chow intake $\left(F_{(2,77)}, 56.172, p=0.000\right)$, and this effect was modified by a time $\mathrm{x}$ access interaction $\left(F_{(4,152)}: 17.786, p=0.000\right)$, reflecting the differing patterns of change across the experiment shown by mice in different access conditions. Control mice increased their chow intake from week 1 to week $6(p=0.000)$, 
with no further changes at week $9(p>0.05)$, whereas mice in the DAILY group reduced their chow intake from week 1 to week $6(p=0.000)$, following access to HFD, with no further changes from week 6 to week $9(p>0.05)$. The INT groups did not alter their intake of chow on access days over time, but maintained the levels seen during week 1. These patterns of change in intake over time resulted in mice in the control group eating the most calories from chow, followed by the INT group, and finally the DAILY group, with the latter having the lowest chow intake of all three access groups (all pairwise comparisons $p<0.05)$.

Chow intake on non-access days (Figure 4D).

In contrast to the lack of an effect of genotype on chow intake on access days, on non-access days, GHSR null INT mice consumed less chow than WT INT mice (main effect of genotype: $\left.F_{(1,30)}: 6.844, p=0.014\right)$. This effect was also modified by time (time by genotype interaction: $\left.F_{(2,60)}: 3.175, p=0.044\right)$. GHSR null mice on the INT schedule reduced their intake of chow after the first week of limited access and ate less chow than WT mice at both week $6(p=0.017)$ and week $9(p=0.014)$. 


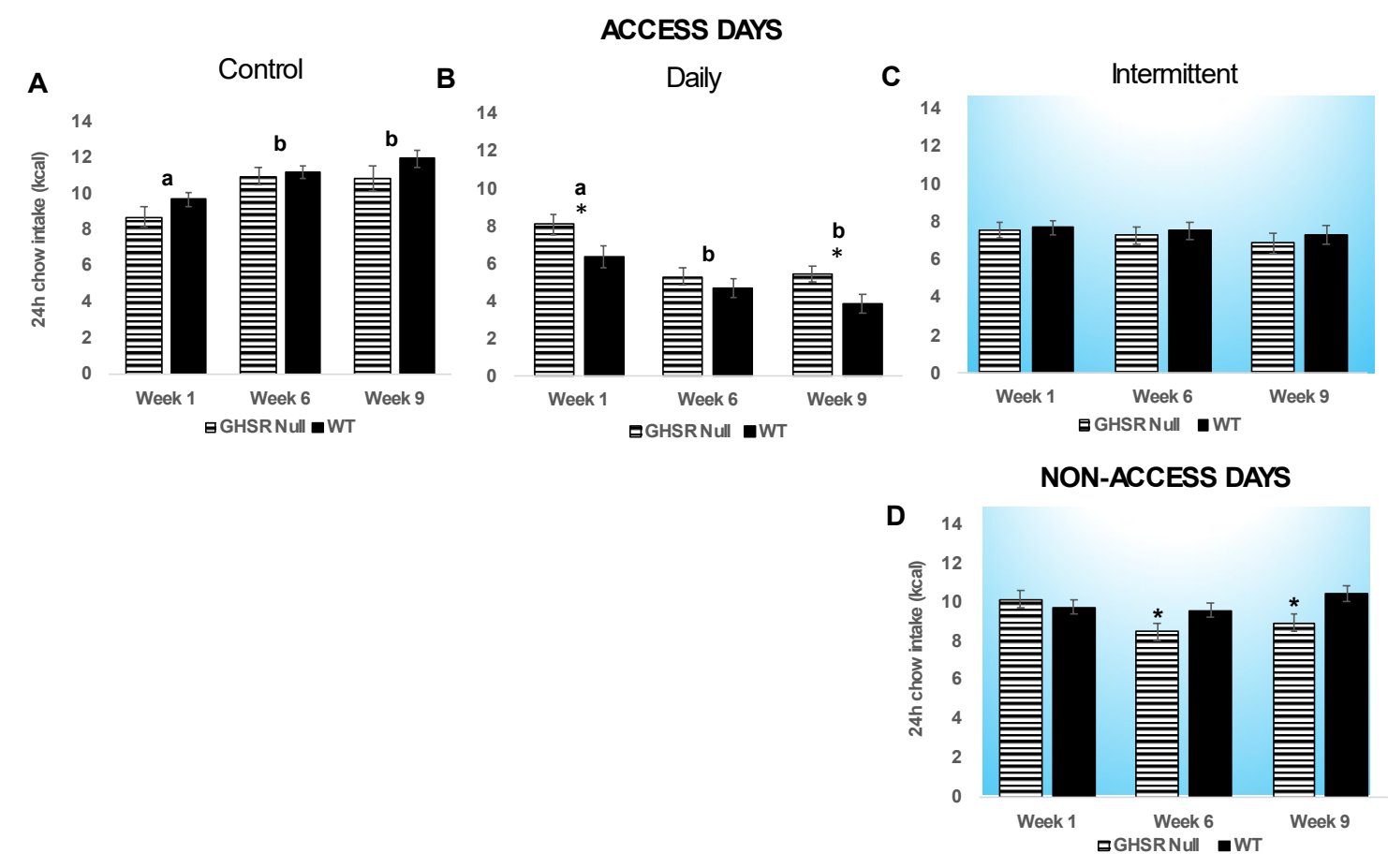

Figure 4. Average 24h chow intake. A) Control mice moderately increased their chow intake from Week 1 to Week 6. No further changes were observed from Week 6 to Week 9 (a,b, $p<0.05)$. B) DAILY mice reduced their intake of chow from Week 1 to Week 6 , with no further changes from Week 6 to Week 9 (a,b, $p<0.05)$. C) INT mice did not alter their intake of chow, despite having access to an HFD on MWF. D) INT GHSR null mice decreased their intake of chow on non-access days $(* p<0.05)$.

24 hour intake on access and non-access days (Figure 5).

Caloric Intake on Access days.

Consistent with our earlier studies, INT mice had a higher $24 \mathrm{~h}$ caloric intake on access days than mice in the other two groups, but whereas INT mice consumed more on access days than the control group at all time points, they only ate more than those in the Daily group from Week 6 onwards (time*access interaction: $F_{(4,132)}=2.592, p=0.039$, pairwise comparisons $p<0.05$ ). By the end of the experiment (week 9), WT male mice were consuming more total calories than male GHSR null mice (time*genotype*sex interaction: $\left(F_{(2,132)}, 3.487, p=0.033\right)$ and male WT mice were eating more calories than female WT mice. 
Caloric Intake on non-access days.

On non-access days GHSR null mice in the INT group consumed fewer calories than the WT INT mice during Weeks 6 and 9 (time* genotype*access interaction: $F_{(4,132) \text { : }}$ 3.158, $p=0.016$, pairwise comparisons $p=0.017$ and $p=0.007$, respectively). Consistent with the decrease in chow intake in the INT group seen on non-access days, and as shown in Figure 5, DAILY group intake as a whole, was consistently higher than the INT groups at all time points (main effect of access: $\left(F_{(2,66): 48.3,25, p}=0.000\right)$.

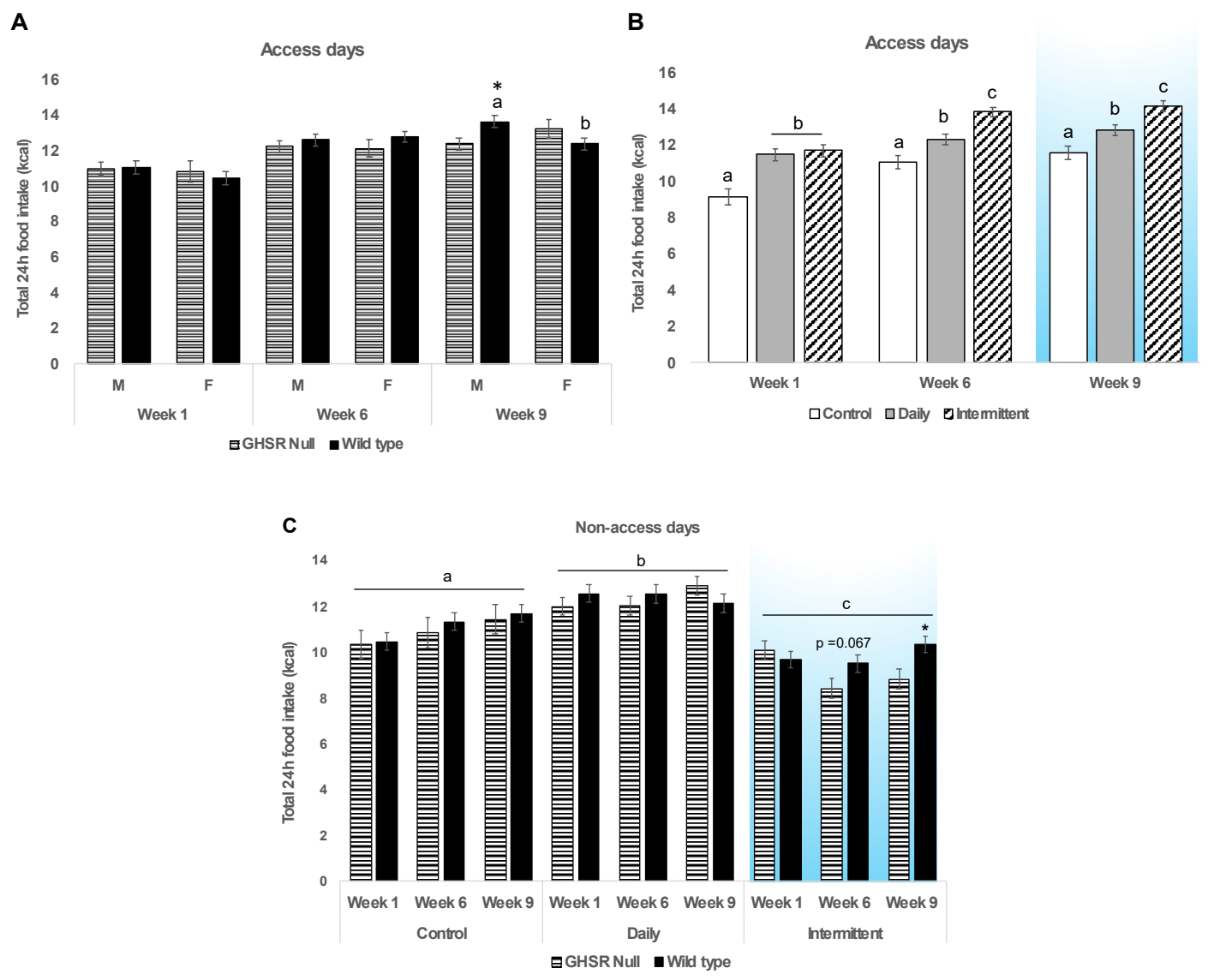

Figure 5. 24h total caloric intake. Top row depicts total calories consumed on days that INT mice had access to HFD. A) At the end of the study, male WT mice consumed more total calories than male GHSR null mice $(*, p<0.05)$, and male WT mice consumed a greater amount than female WT mice (a,b, $p<0.05)$. B) DAILY and INT mice consume more calories during the first week of binge access (a,b, $p<0.05)$, and subsequently, INT mice eat more than both control and DAILY mice for the remainder of the study (a,b,c, denotes heterogenous groups, $p<0.05$ ). INT mice also consume 
more calories overall, than any other group in the study on access days. C) Intermittent mice ate the least on non-access days (T,Th,Sat, and Sun). During Week 6, INT GHSR null mice showed a trend towards consuming fewer total calories than INT WT mice, and by the end of the study, this difference was significant $(*, p<0.05)$.

Cumulative intake for each phase of the study (Figure 6).

During the baseline period, all mice consumed similar amounts of standard chow $(p>0.05$ for all factors; Figure 6A). By the end of the 6 week HFD access period, DAILY mice consumed the greatest amount of calories (main effect of access: $F_{(2,88)}$ : $9.768, p=0.000)$, and differed significantly from both INT $(p=0.000)$ and control mice $(p=0.000)$. INT and control mice consumed a similar amount during this period $(p>$ 0.05). Finally, during the last two weeks of the study, following stereotaxic surgery to reinstate GHSR in CRE AAV mice, a significant genotype by access interaction was

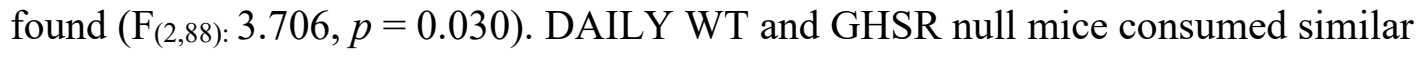
amounts of food, however, if receiving INT access to HFD GHSR null mice consumed fewer calories than WT mice $(p=0.013)$. No significant effects of reinstatement were found.

A

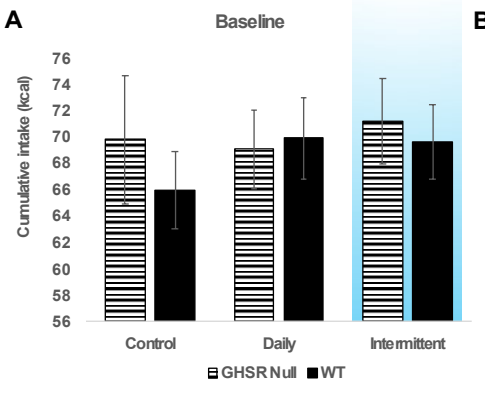

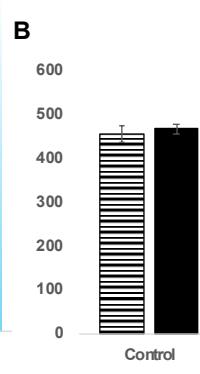

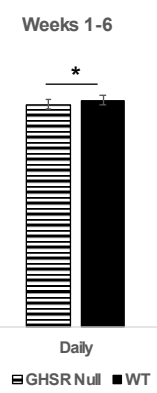

C

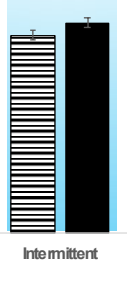

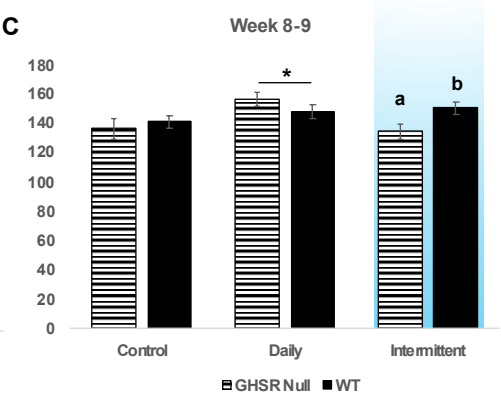

Figure 6. Cumulative intake during each of the three main phases of the experiment. Daily mice consumed the greatest amount of food over the course of the 6-week binge period, as well as during the final phase of the study $(*, p<0.05)$. In the last two weeks, there was a genotype by access interaction, such that GHSR null mice ate less than WT mice, but only in the INT group (a,b; $p<0.05)$. 
Body weight (Figure 7).

At the end of the baseline period, GHSR null mice weighed less than WT mice $\left(F_{(1,89)}=3.973, p=0.050\right)$, and female mice weighed less than male mice $\left(F_{(1,89)}=\right.$ $42.187, p=0.000$ ). For the remainder of the experiment, body weight was analyzed in terms of weight change (calculated as change relative to the baseline for week 1, relative to week 1 for week 6 , and relative to week 6 for week 9). After the first week of HFD exposure, GHSR null mice had gained less weight than WT mice $\left(F_{(1,88)}=5.460, p=\right.$ $0.022)$ but there was no significant effect of either sex, or access condition $\left(F_{(1,88)}=\right.$ $1.942, p=0.150 ; F_{(1,88)}=0.147, p=0.703$ respectively). Mice in the DAILY group gained more weight than those in the other two groups during the next 5 weeks of access (main effect of access condition $F_{(2,88)}=10.792, p=0.000$ ), whereas control and INT mice did not differ $(p=0.146)$. In the final 3 weeks following surgery female mice gained less weight than male mice $\left(F_{(1,88)}=4.505, p=0.038\right)$, however, there were no effects of either genotype $\left.F_{(1,88)}=0.091, p=0.763\right)$, access $\left(F_{(1,88)}=0.618, p=0.542\right)$, or virus $\left(F_{(1,88)}=0.039, p=0.844\right)$.

A

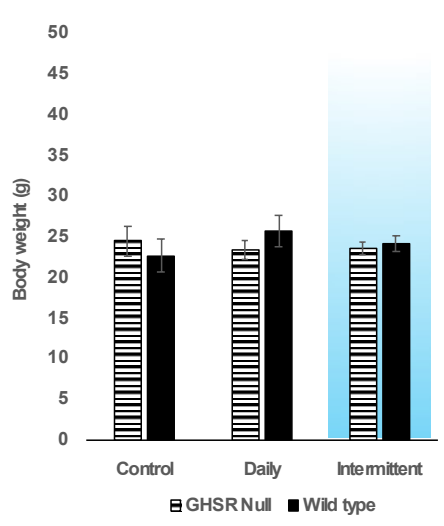

B

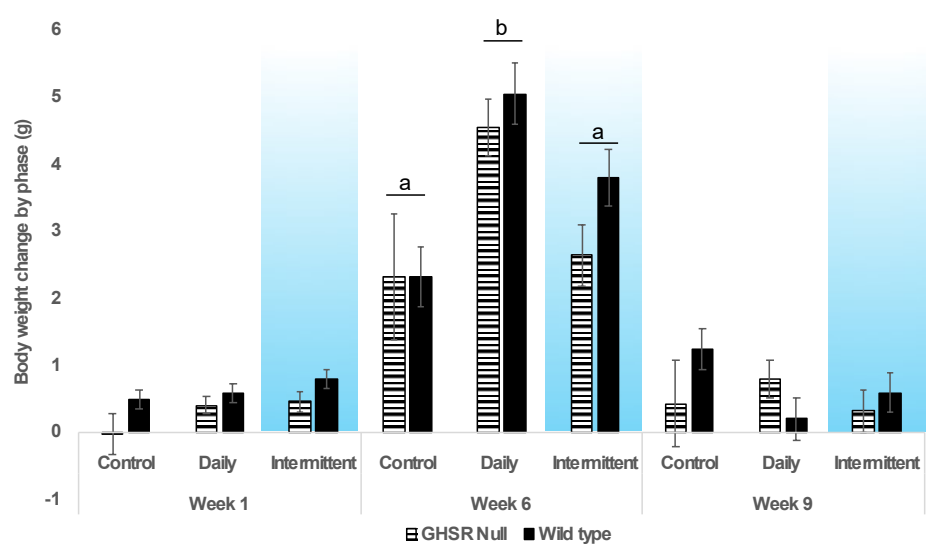

Figure 7. Body weight. A) At baseline, GHSR null mice weighed less relative to WT mice and females also weighed less than males. B) By the end of the first week of HFD 
access, WT mice had gained more weight than GHSR null mice $(\mathrm{p}<0.05)$. By the end of week 6 of the binge paradigm, daily mice had gained the most weight, as compared to both control and INT groups $(a, b, p<0.05)$. No group differences were found at week 9 , all mice had gained a similar amount of weight during the last 3 weeks of the study.

\section{Experiment 2.4. Antagonism of the GHSR in the VTA to attenuate HFD following the establishment of binge-like intake.}

Materials and Methods.

Animals.

Adult male C57BL6/J mice $~ 3$ months of age, purchased from Charles River

(Charles River, St. Constant, QC) and weighing 22-25g were used in this study $(n=36)$.

Housing, baseline diet, and habituation to HFD were as described in the previous

experiment. Mice were assigned to 3 access groups: CONTROL $(n=12)$; DAILY $(n=12)$;

and INT $(n=12)$, and limited access conditions imposed as described above.

After 3 weeks of limited access model exposure, mice underwent surgical

implantation of a unilateral cannula directed at the VTA accompanied by subcutaneous

implantation of an osmotic mini-pump delivering either saline or the GHSR antagonist

(JMV 2959; 5ug/day) at a controlled rate (see 'Drug and osmotic mini-pump', below).

Mice remained in their respective access conditions for an additional 3 weeks to

determine the effects of blockade of GHSR in this region on established high-fat

bingeing. Following the last binge, mice were perfused transcardially and brains were

fixed and stored for further processing.

Surgical procedures.

General surgical procedures were as described above, but in this experiment, a

unilateral cannula $(0.15 \mathrm{~mm}$, stainless steel; $5 \mathrm{~mm}$ in length $)$ connected to an osmotic

mini-pump was implanted. Once the coordinates (AP: -2.95; ML: 0.7; DV: -4.55) were

located, a 22-gauge needle was used to drill a hole through to the surface of the brain. 
The cannula was then lowered and secured in place using acrylic and dental cement. Once the cement was dried and the cannula securely in place, the surrounding skin was sutured gently to prevent the development of infection. A small incision was made in the skin above the intrascapular space and an osmotic mini pump (at a flow rate of $0.24 \mathrm{ul}$ per hour) delivering either 5ug/day of JMV-2959 (antagonist) or a vehicle solution ( $0.9 \%$ saline) was inserted subcutaneously. The second incision was sutured and the application of local antibiotics (Polysporin) and topical analgesic (Xylocaine jelly, 2\%), aided in the prevention of infection and provided pain relief, respectively.

The mice were moved post-operatively into a clean cage and placed onto a heating pad $\left(37^{\circ} \mathrm{C}\right)$ to assist in maintaining an appropriate body temperature. The cage contained Hydrogel ${ }^{\mathrm{TM}}$ for hydration purposes (Animal Specialties and Provisions, Quakertown, PA) and a piece of paper towel laid over cob bedding (to prevent inhalation of bedding). The mice were monitored regularly and once awake, were moved back into their housing room. To aid with recovery, mice were monitored closely and given a $1 \mathrm{mg} / \mathrm{kg}$ dose of Meloxicam subcutaneously daily for 2 to 3 days to provide pain relief. Topical analgesic was used to treat any minor scratches ( $2 \%$ xylocaine jelly). Termination of post-operative monitoring was determined by Carleton University ACVS staff and study personnel. Mice continued to receive HFD during the acute post-operative period (on the same schedule as prior to surgery) to avoid potential weight loss or avoidance of chow due to previous HFD exposure, which could potentially slow recovery.

Drug and osmotic mini-pumps.

The GHSR antagonist, JMV-2959 [(R)-N-(2-(1H-indol-3-yl)-1-(4-(4methoxybenzyl)-5-phenethyl-4H-1,2,4-triazol-3-yl) ethyl)-2-aminoacetamide 
hydrochloride] was purchased from EMD Millipore (Product \#345888; Calbiochem). The dose of JMV 2959 used in this study was chosen following an examination of the literature (Prieto-Garcia et al., 2015), as well as doses previously used in our lab that demonstrate behavioural efficacy on a number of variables. JMV-2959 was dissolved in sterile $0.9 \%$ saline and injected into osmotic mini-pumps for delivery into the VTA (Alzet, Model 1004 (mouse); 28-day delivery; Durect Corporation, Cupertino, CA). Sterile saline was used as a vehicle solution and the mini-pumps were filled in an identical manner.

Perfusion and Immunohistochemical analysis.

Upon conclusion of behavioural testing, mice were injected with a lethal dose of Dorminal (1mg/kg i.p.; CDMV, Quebec, Canada) and perfused transcardially with $0.9 \%$ saline, followed by $4 \%$ paraformaldehyde. Brains were then extracted and post-fixed in $4 \%$ paraformaldehyde for $24 \mathrm{~h}$, followed by submersion in a $30 \%$ sucrose solution $(\mathrm{w} / \mathrm{v})$ prior to sectioning. $40 \mu \mathrm{m}$ sections containing the VTA (Figs. 55-63, Paxinos and Franklin, 2001) were sliced at $-21{ }^{\circ} \mathrm{C}$ on a Thermoscientific Cryostat. Sections were then analyzed under a light microscope to determine the location of cannula placements. All mice with evidence of misplaced cannula placements were excluded from statistical analysis (see Figure 8 for a representative photomicrograph of a representative on target cannula placement).

\section{Statistical analyses.}

Data were analyzed using mixed two-way repeated measures ANOVA using three time points as the within-subjects factor: the pre-surgery HFD access period (last 2 days of the binge period), the early post-surgery period (first 3 days of the post-surgery period) and the end of the post-surgery period (last 2 days). Drug and access schedule $(2 \times 3)$ 
served as the between group factors. Follow up multiple comparisons using Fisher's LSD were performed when necessary. Data from this experiment are expressed as mean \pm standard error of the mean (SEM). All statistical analyses were performed with SPSS 20.0 for Macintosh (IBM Corp., Armok, NY) and statistical significance was set at $\alpha$ $=0.05$.

Results.

Cannula placement verification (Figure 1).

Cannula placements were verified using standard light microscopy, and any mice that had cannulae located outside of the VTA were removed from the data set and subsequent statistical analysis $(n=11)$. Due to some difficulty targeting the VTA with an indwelling cannula without generating excessive bleeding, co-ordinates had to be adjusted slightly, resulting in an increased percentage of missed cannulae.

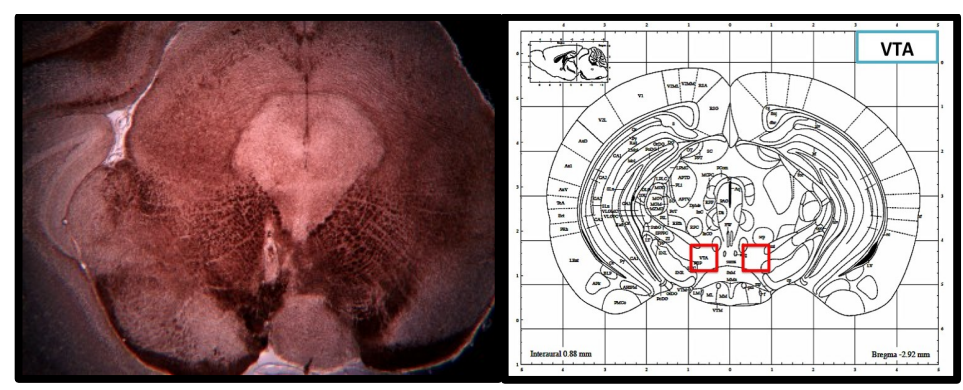

Figure 1. Cannula placement verification. Representative photomicrograph illustrating location of a cannula through which either saline or the GHSR antagonist JMV-2959 were infused, via osmotic mini-pump. Unilateral cannulae were aimed at the VTA (co-ordinates: AP: -2.95; ML: 0.7; DV: -4.55) On the right is a figure from Paxinos and Watson (2001) demonstrating the target location.

Effect of genotype and access condition on binge behavior (Figure 2A).

Mice in both DAILY and INT access groups showed bingeing behaviour by eating $25 \%$ or more of their daily baseline daily caloric intake during the $2 \mathrm{hr}$ access to HFD during the pre-surgery binge period. Similarly, mice in both groups increased binge size after surgery (within subjects effect of time: $F_{(2,38)}=4.597, p=0.016$; pre vs. early 
post: $p=0.005$; pre vs. late post: $p=0.18$. No changes were observed from early postsurgery, to late post-surgery $(p>0.05)$. Further, neither access $\left(F_{(1,19)}=.136, p=0.491\right)$ nor drug $\left(F_{(1,19)}=.545, p=0.545\right)$ influenced this measure of HFD intake.

Average HFD intake during the $2 \mathrm{~h}$ access period (Fig $2 B$ ).

The increase in binge size resulted in a greater caloric intake of fat from the presurgery period to the early post-surgical period for both DAILY and INT mice, regardless of drug condition (within subjects effect of time, $F_{(2,38)}=6.091, p=0.005$; pre vs early post: $p=0.003$; pre-vs late post: $p=0.011$; early post vs. late post: $p>0.05$ ). HFD diet intake was not affected significantly by drug or access condition or their interactions.

A

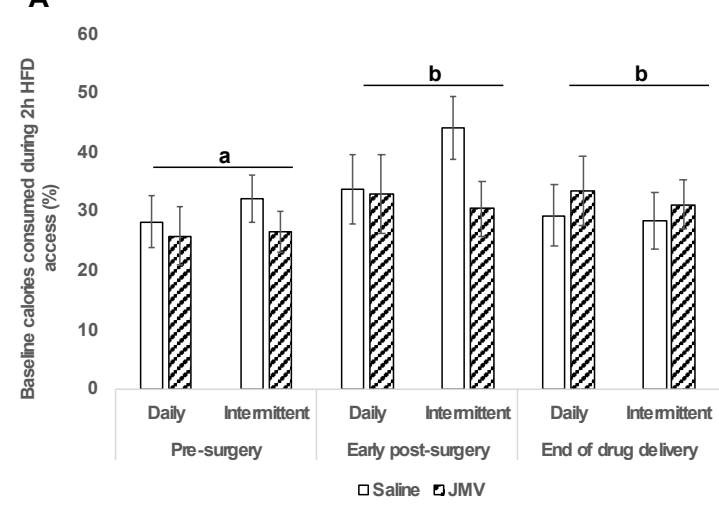

B

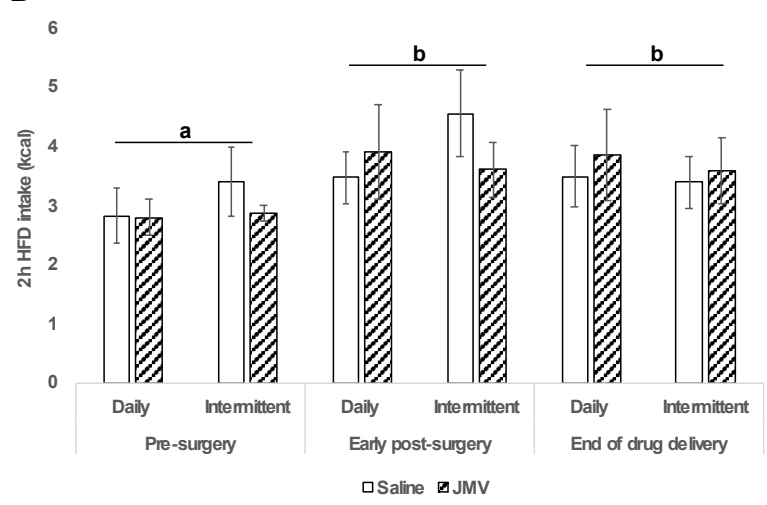

Figure 2. High fat diet intake. A) Percent of baseline kilocalories consumed from HFD during $2 h$ access. All mice increased their intake of HFD from pre- to early post-surgery, with no further increases by the end of the study ( $a, b$, denote differences between access groups; $\mathrm{p}<0.05$ ). No differences were observed between DAILY and INT mice. B) Kilocalories consumed from HFD during $2 \mathrm{~h}$ access. All mice increased HFD intake from pre- to early post-surgery, with no further increases by the end of the drug delivery period.

Effect of access and drug condition on chow intake (Figure 3).

Chow intake on access days.

Baseline intake of chow was similar for all mice prior to initiation of the limited access model (data not shown). Mice altered their chow intake over time depending on 
frequency of access to HFD (time by access interaction: $F_{(6,81)}=4.579, p=0.000$ ). By the end of the pre-surgery period, mice in the DAILY group had reduced their intake of chow, consuming significantly fewer calories from chow than both control and INT mice, whereas intake between INT mice and control mice did not differ (see Figure 2A). This pattern continued for the remainder of the study, with DAILY mice consuming less chow, as compared to the INT and control mice (all pairwise comparisons $p<0.05$, see Figure 2C). Following surgery, all mice increased their intake of chow slightly (within subjects effect of time: $\left.F_{(1,27)}=14.116, p=0.001\right)$, however DAILY mice consumed the fewest calories from chow (panel C), relative to both control (panel B) and INT mice (panel D) (main effect of access: $F_{(1,27)}=19.129, p=0.000$ ). There were no effects on JMV-2959 administration on this measure.

\section{Chow intake on non-access days.}

As in the previous experiment, non-access days pertain only to mice in the INT

conditions, and as such, Figure 2E depicts the analysis for this experimental group only (as a function of time [pre-surgery] and drug [post-surgery]). INT mice exhibited a gradual and modest increase in chow intake over the course of the study on days that they did not receive HFD (time: $F_{(2,24)}=4.725, p=0.019$ ); pre-surgery intake did not differ from the early post-surgical period, but intake was higher by the end of the study ( $p=$ 0.029). There were no statistically significant effects of drug on chow intake at any of the chosen time points, with mice in the JMV and saline groups showing comparable intake. 


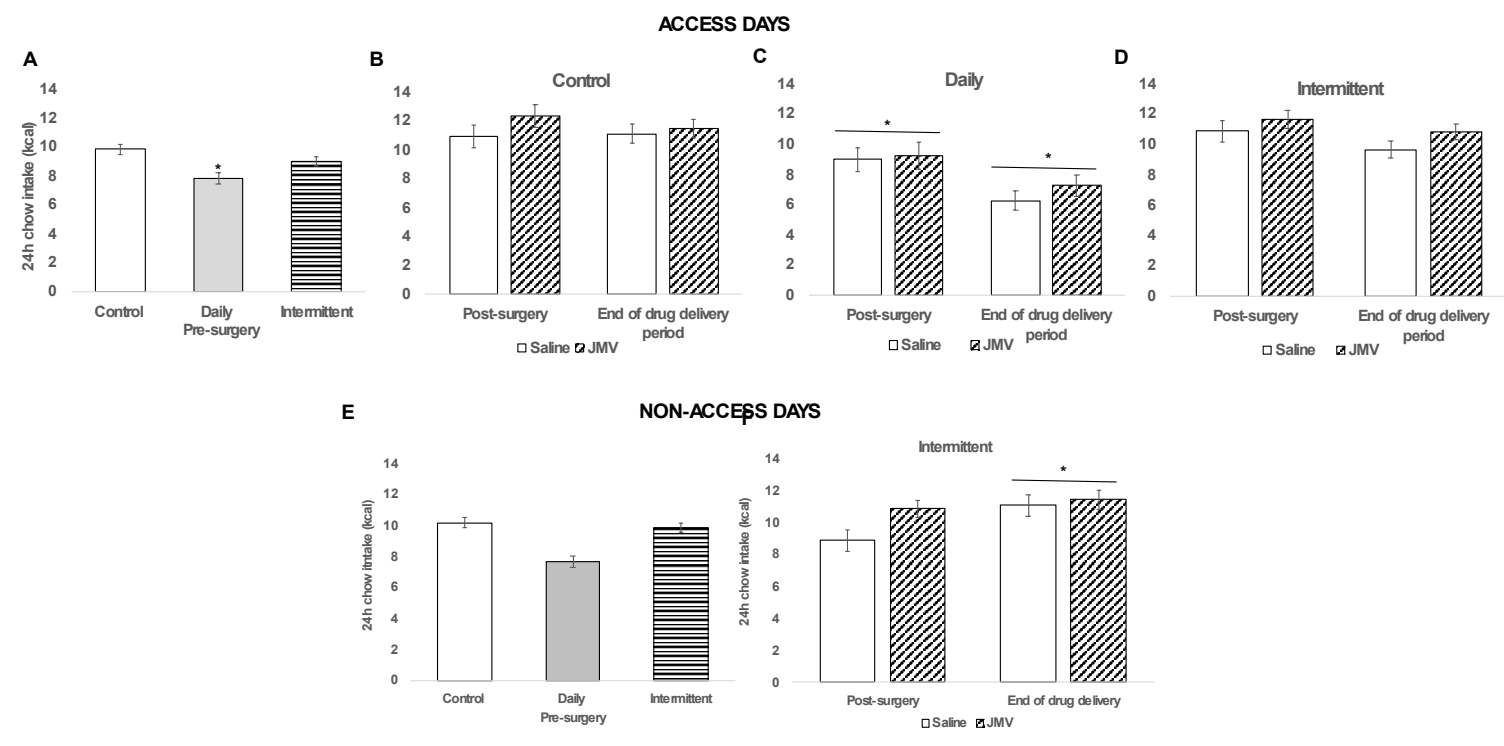

Figure 3. 24h chow intake. Access days: A) On access days, DAILY mice consumed the least amount of chow prior to surgery. B) Following surgery, DAILY mice continued to eat the least amount of chow on access days, as compared to C) control and D) INT mice. There was no effect of JMV administration on chow intake. Non-access days: E) On nonaccess days, mice showed a similar pattern of intake as on access days. F) INT mice consumed a bit more chow by the end of the study (as compared to pre-surgery). There was no effect of JMV administration.

$24 \mathrm{~h}$ daily intake on access days (Figure 4A).

Scheduled access to HFD had an overall effect on total caloric intake $\left(F_{(1,30)}=\right.$

$46.334, p=0.000)$, however, this effect was modified by time $\left(F_{(3.90)}=3.847, p=0.002\right)$.

Control mice exhibited stable intake over time, whereas DAILY mice increased their intake post-surgery. Mice in the INT group increased their caloric intake over time and ate significantly more than both control and DAILY mice at all time points (all pairwise comparisons $p<0.05$ ). As found in our previous studies, INT access to the HFD induced a binge-like profile of intake, which resulted in greater intake on days when HFD was available. 
$24 \mathrm{~h}$ daily intake on non-access days (Figure 4B).

During the early post-surgery period, mice in the INT group reduced their total caloric intake if they received saline, but continued to eat a similar amount of calories if they received JMV-2959 (time by drug interaction: $F_{(1,12)}=5.58, p=0.036$ ). There was no effect of drug independent of time $\left(F_{1,12)}=3.775, p=0.076\right)$.

ACCESS DAYS

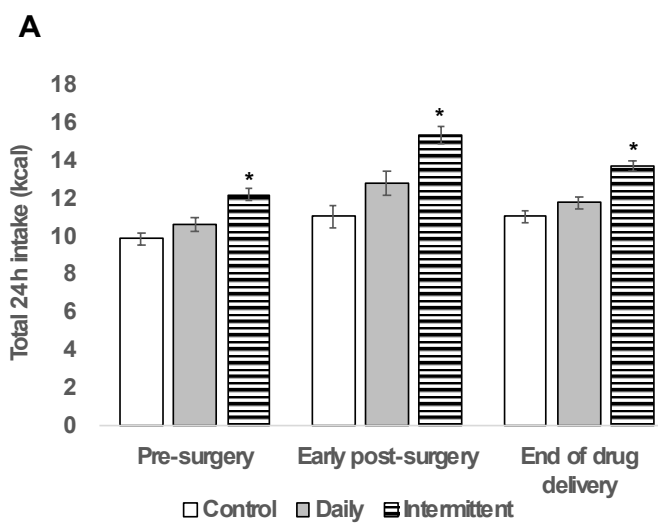

NON-ACCESS DAYS

B

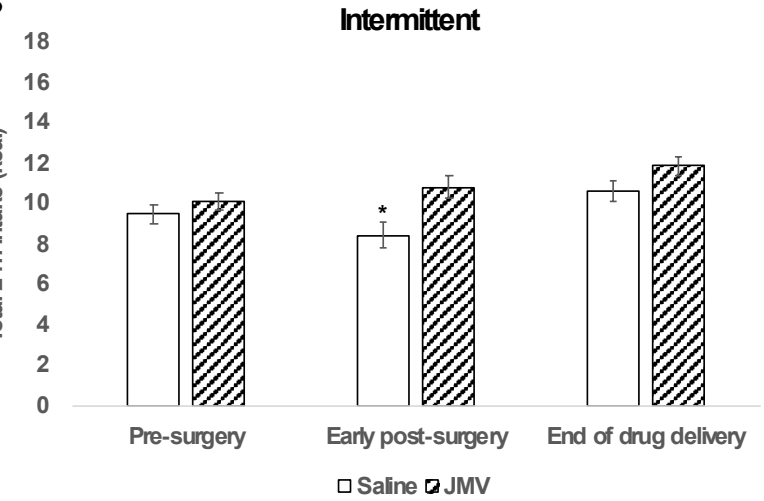

Figure 4. Total 24h total caloric intake. A) On access days, INT mice consumed a greater number of calories over the $24 \mathrm{~h}$ period on access days than either control or DAILY mice $(*, p<0.05)$. B) On non-access days, INT mice consumed fewer calories during the early post-surgery period if they received saline $\left({ }^{*}, p<0.05\right)$. No other changes were observed.

Cumulative intake and dietary composition over the pre-and post-surgery periods (Figure 5).

High fat diet.

Cumulative intake of HFD over the course of the study was greater during the post-surgery period, than during the pre-surgery period $\left(F_{(1,19)}=23.120, p=0.000\right)$. No effects of access $\left(F_{(1.19)}=0.120, p=0.733\right)$ nor $\operatorname{JMV}\left(F_{(1.19)}=0.121, p=0.732\right)$ were observed. In other words, it did not matter how often mice received access to the HFD or whether they received JMV-2959, they consumed a similar amount of HFD during each of these two phases of study. 
Chow.

All mice increased their intake of chow from pre- to post-surgery $\left(F_{(1,27)}=\right.$ $509.050, p<0.05)$. However, mice in the INT group increased the amount of chow they ate post-surgery to a greater extent than DAILY mice (time by access interaction: $F_{(2.27)}=$ $4.451, p=0.010)$. Overall, DAILY mice consumed less chow than INT mice $\left(F_{(2,27)}=\right.$ 15.646, $p=0.000$ ). Mice that received JMV-2959 exhibited a trend towards eating more chow than SAL treated mice, however, this effect did not reach statistical significance $\left(F_{(2,27)}=3.940, p=0.057\right)$.

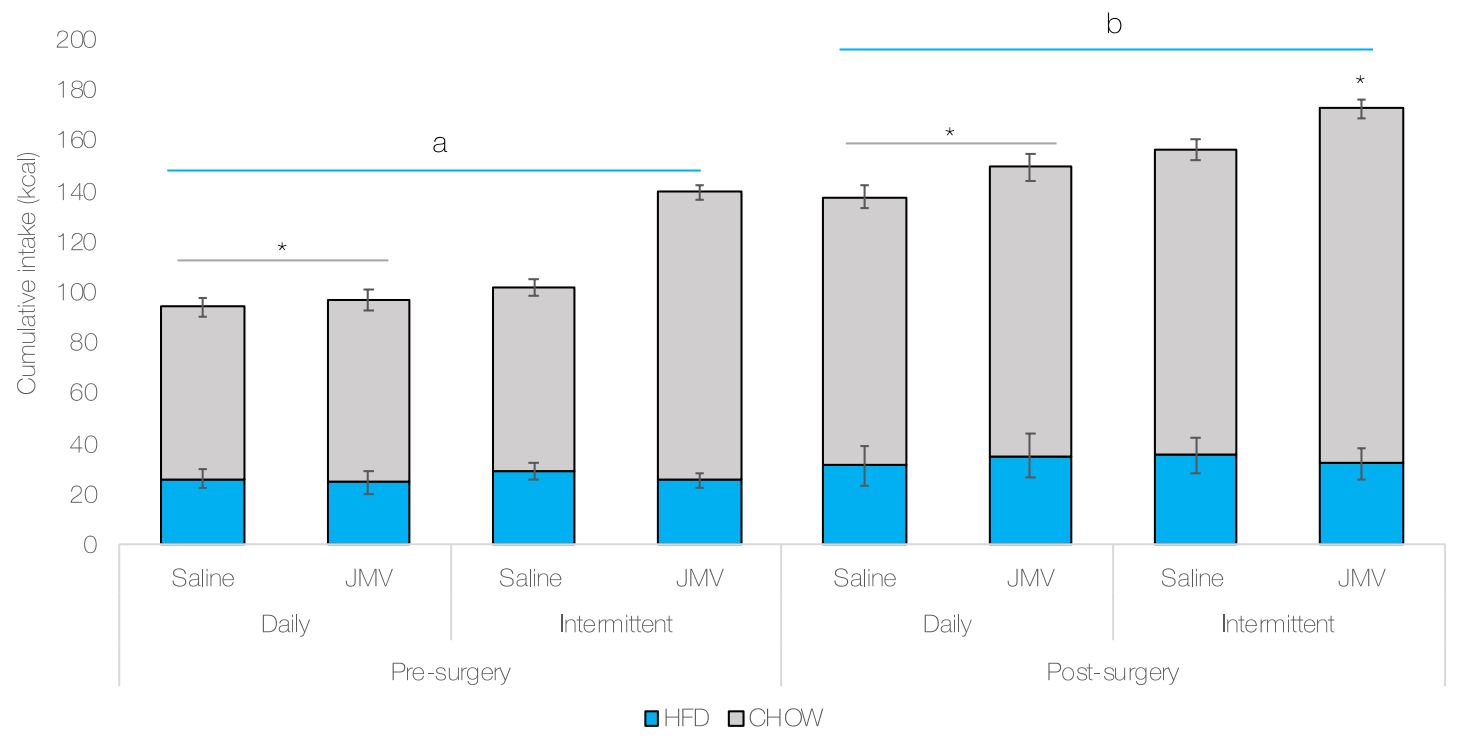

Figure 5. Dietary composition. All mice consumed more HFD during the post-surgery period, relative to the pre-surgery period $(\mathrm{a}, \mathrm{b}, \mathrm{p}<0.05)$. Daily mice ate less chow than INT mice over both periods $(*, p<0.05)$. Mice administered JMV into the VTA exhibited a trend towards consuming more chow in the intermittent group post-surgery $(p=0.056)$.

Cumulative intake for each phase of the study (Figure 6).

A few of the main concerns with the binge eating of energy-dense foods, apart from the psychological distress experienced in humans, are weight gain, accumulation of adipose tissue, and/or metabolic syndrome associated with repeated overconsumption. As such, we were interested in analyzing net caloric intake over the different phases of the 
experiment, to determine if outcomes differed as a function of access group and/or GHSR antagonism in the VTA. All mice ate a greater number of calories during the post-surgery phase $\left(F_{(1,27)}=580.191, p=0.000\right)$, however, a marginal time by drug interaction was also found, such that mice receiving JMV into the VTA consumed more cumulative calories than mice receiving saline $\left(F_{(1,27)}=4.122, p=0.052\right)$. Although the p-value was above our stated cut-off for statistical significance, we mention it as an effect here, mainly because mice were not receiving the antagonist until the post-surgical period. Therefore, any effect of drug would be expected to emerge only during the latter half of the study. We also found main effects of both access $\left(F_{(2,27)}=12.935, p=0.000\right)$ and $\operatorname{drug}\left(F_{(1,27)}=8.041, \mathrm{p}=0.009\right)$ on cumulative caloric intake. INT access to HFD resulted in increased caloric intake, relative to both DAILY and control mice $(p<0.05)$. Control and DAILY mice ate a similar number of calories, indicating that INT access was the only HFD regimen able to induce net overconsumption. Overall, mice that received JMV2959 ate more cumulatively than mice receiving saline $(p=0.011)$, which is counter to our hypothesis, given that GHSR antagonism typically results in decreased food intake.
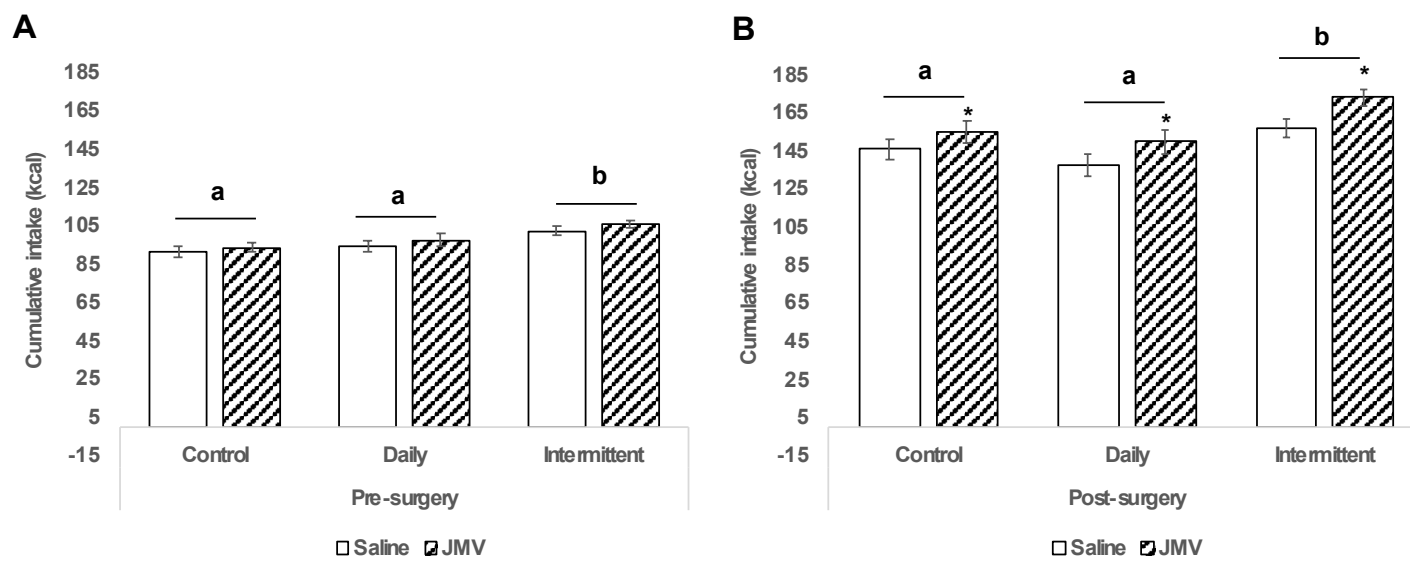

Figure 6. Cumulative caloric intake. Mice consumed fewer calories during the presurgery phase of the study (A) than during the post-surgery period (B), both three weeks in length. INT mice consumed a greater number of calories over both the pre- and post- 
surgery periods, as compared to both control and DAILY mice, who consumed a similar amount of cumulative kilocalories (a,b: $p<0.05)$. Mice receiving JMV-2959 had higher cumulative caloric intake than mice receiving saline vehicle solution following surgery.

\section{Body weight (Figure 7).}

During the baseline period, mice gained minimal weight and there were no group differences (data not shown). During the pre-surgical period there were no differences in body weight gain between the access groups $\left(F_{(2,32)}=1.361, p=0.232\right.$; see Figure 7$)$.

However, after 3 additional weeks of high-fat access, with either JMV or SAL infusion into the VTA, DAILY and INT mice gained more weight than controls (main effect of $\operatorname{access}\left(F_{(2,32)}=4.304 ; p=0.024\right)$. There were no effects of $\operatorname{drug}\left(F_{(1,32)}=1.158 ; p=0.291\right)$ and no interaction between drug and access $\left(F_{(2,32)}=0.59 ; p=0.61\right)$.
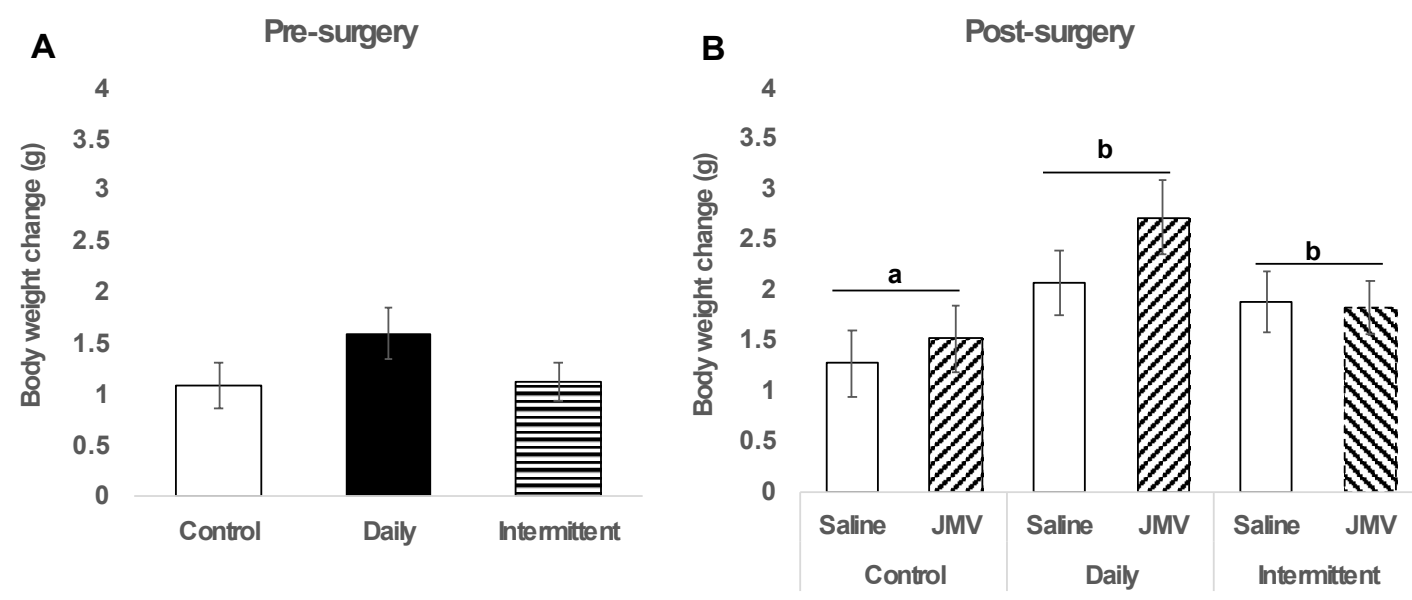

Figure 7. Body weight. A) Pre-surgery. Mice gained modest and similar amounts of weight during the pre-surgery period. B) Post-surgery. During the 3 weeks following surgery, both DAILY and INT mice that received access to the HFD gained a greater amount of weight than control mice. (a,b, $p<0.05)$.

\section{Discussion.}

There is a risk of metabolic disturbances, as well as psychological distress in individuals suffering from BED and understanding the neurochemical pathways that contribute to this disorder is an important step in understanding their etiology, as well as 
potential therapies. We conducted the current studies in order to test the hypothesis that the GHSR, which is present in mesolimbic regions such as the VTA (Guan et al., 1996; Abizaid et al., 2006), is important for the development of binge-like intake of a diet high in fat.

In the current experiments, as in our earlier studies, we used the limited access model to elicit binge-like consumption of HFD in mice. However, here we sought to extend our previous findings that mice lacking the GHSR exhibited a reduced propensity to binge on fat relative to WT mice, by exploring the possibility that GHSR signaling specifically within the VTA is important for the full expression of binge-like consumption of HFD. First, we sought to replicate the findings described in Chapter 3 showing that GHSR KO mice demonstrate reduced binge-like intake of HFD, using a different transgenic mouse that also lacked the GHSR (Zigman et al., 2005) and to determine whether rescue (i.e. transcriptional reinstatement) of the GHSR in the VTA would restore HFD bingeing to levels seen in WT mice. In the second study, we used a pharmacological approach to investigate the role of GHSR signaling in the VTA on binge eating by administering a specific and competitive GHSR antagonist JMV-2959 chronically into the VTA.

Overall, we were able to replicate a number of components of the limited access model in these studies and demonstrated that short access to a high-fat diet induces bingelike intake in mice. Mice given limited and INT access to the HFD diet developed a binge-compensate pattern of intake, with increased calories consumed on access days and reduced caloric intake on the following days. Mice in the second study, however, did not compensate significantly (see Figures 5, Study 4.1 and 3, Study 4.2), perhaps due to their 
reduced binge size relative to previous cohorts of mice. In all mice, access to HFD resulted in binge-like intake when available, and reduced intake of chow when access occurred daily. Mice that received access to the HFD also consumed a greater number of calories cumulatively, which, over extended periods, may result in a chronic positive energy balance leading to weight gain or metabolic disturbances. Therefore, this model was able to induce binge-like feeding in mice, as seen previously (King et al., 2016).

Contrary to our hypothesis, as well as our previous findings, we did not find a robust reduction in high-fat bingeing in GHSR null mice. Despite this, we did observe a genotype by sex interaction, such that female GHSR null mice binged less on the HFD than WT females. We chose to include both male and female mice in the first experiment, as binge eating disorder is more prevalent in females (Smink et al., 2012), but it is also the most common eating disorder seen in males. It is possible that our results reflect an estrogenic modulation of GHSR responsivity in the females that is lost when they sustain global GHSR knock-out. Frazao and colleagues (2014) have shown that heightened levels of estrogen increase GHSR mRNA expression in the hypothalamus, and if this is also true in the VTA, we may be seeing reduced consumption of a palatable diet in GHSR null females due to a lack of upregulation of the receptor. Precisely which aspect of ingestion is altered in the GHSR null female mice is not clear, but meal pattern dynamics/intermeal intervals (Espinosa-Carrasco et al., 2018), rates of consumption (Keisler et al., 2017), and/or liking responses (Lardeux et al., 2013) are all possibilities.

The lack of robust genotype effects found in the current study, as compared to our earlier findings, may stem largely from the differing genetic backgrounds of the mice, as they differ from one another, as well as from the GHSR KO mice used in the previous 
study. To minimize the influence of strain differences on bingeing behaviour, all of the mice, despite their differing transgenic nature, were backcrossed onto a C57BL6 background strain. However, introduction of transgenes or mutations often result in pleiotropic 'off-target' effects or induce compensatory mechanisms, particularly during development (Wolfer and Lipp, 2000). In future studies, improvements could be made by exploring the phenotype of mutant mice using various complementary assays within a given behavioural domain (for example: high-fat bingeing) (Bailey et al., 2006). While the rescue portion of our experiment would have provided strong evidence that the mutation is indeed responsible for the behavioural outcome, the effects prior to reinstatement were only trends or interactions with other variables and therefore, the effects of rescue were negligible on our outcome measures.

We found no effects of restoration of GHSR in the VTA on high-fat bingeing. Clearly, the lack of significant differences in bingeing behaviour between the GHSR-null and WT mice would be expected to contribute to the lack of influence of GHSR rescue on high-fat bingeing, however, this may also be attributed to the incomplete rescue of the receptor in the VTA. GHSR mRNA levels only reached about $30 \%$ of that seen in WT mice, and given that GHSR restoration, although neuron-specific, was not directed at TH neurons exclusively, it could be transducing neurons of a glutamatergic or GABAergic phenotype, which could weaken any overall effects. Had the rescue been more complete, and specific to TH cells (as in Chuang et al., 2011), we might have seen a more robust increase in HFD intake in the GHSR rescued mice. Chuang et al. (2011) used TH-cre driver lines to reinstate GHSR specifically in catecholaminergic neurons, however their rescue was brain wide, whereas we focused exclusively on the VTA. Whether the genetic 
nature of the mice underlies the differences between experimental findings is unclear. Thus, although the importance of GHSR activity for enhancing food reward (Valdivia et al., 2014, 2015; MacKay et al., 2016; Chuang et al., 2011) through its interaction with DA cells in the VTA, which enhances DA output in the NAc (Abizaid et al., 2006) is well established, it does not seem to be necessary for the expression of bingeing on a high-fat diet under the current experimental paradigm.

Consistent with the lack of effect of GHSR restoration in the VTA, we found that administration of the GHSR antagonist JMV-2959 into the VTA was not sufficient to reduce binge-like intake of HFD once it has already been established. It is possible that the low high-fat consumption in these mice contributed to a lack of effect of JMV-2959, but although some mice did not reach the criteria of $>25 \%$ of baseline caloric intake in the access period it is likely that they did consume $17.5 \%$ in the first hour, which is typical for mice in this paradigm (Valdivia et al., 2014).

The impetus for our hypothesis that GHSR activation in the VTA would play a role in binge-like eating of fat was suggested in part by findings from Bake et al. (2012), who demonstrated that orexigenic NPY and anorexigenic POMC expression were not altered in the ARC $2 \mathrm{~h}$ prior to scheduled HFD feeding. Clearly our data do not support our hypothesis, but they do not rule out a role for GHSR activation in other circuits. Although Bake et al. (2012) did not find alterations in NPY expression, they did not examine GHSR mRNA dynamics, and it is possible that upregulation of GHSRs on NPY neurons, and subsequent second-order inhibition of POMC/CART neurons, may enhance consumption of HFD during a binge in WT mice. Indeed, recent data from our laboratory suggest that GHSR is upregulated in the ARC in anticipation of a scheduled palatable 
meal from Day 4 through to Day 9 of scheduled treat access (Khazall, 2017) and increased sensitivity to ghrelin could enhance intake, via potentiation of NPY/AgRP activity.

In addition to the effect of GHSR activity on binge eating per se, we were also interested in how long-term high fat access would affect caloric intake and body weight, and whether these would be modified by genotype. We found that effects varied across studies, such that in Study 4.1, using GHSR null and WT mice, DAILY mice consumed the largest number of calories cumulatively, as compared to both INT and control mice. As well, GHSR null mice tended to consume fewer calories than WT mice, in the last 3 weeks of the study. Conversely in Study 4.2, we found that INT mice had the highest cumulative caloric intake across the entire study period. Thus, although limited access to an HFD can induce increased caloric consumption over a period of up to 9 weeks, whether INT or DAILY access has the greatest effect is unclear. Despite some minor changes in body weight as a result of HFD access, neither experiment induced a substantial amount of weight gain, despite the fact that mice receiving the HFD exhibited greater net caloric intake in both studies. However, the majority of studies using rodent models of binge eating that do not require stress, or a history of dieting and caloric restriction, do not demonstrate increased body weight as a result of INT HFD (Czyzyk, Sahr, and Statnick, 2010; Corwin et al., 2004; Dimitrou et al., 2000). Therefore, our body weight results are in line with other studies examining bingeing in rodents.

In line with our previous experiments, mice in the INT group did not escalate intake of HFD relative to DAILY mice. Intake of palatable diets during restricted access has been shown to be inversely related to the frequency of access (Corwin et al., 2004; 
Corwin et al., 1998; Dimitrou et al., 2000), as well as to the duration of availability (Kreisler et al., 2017). In our experiments, both groups had the same duration of access but differing intermittency, which may not have been adequate to generate significant differences in fat intake between the two groups.

Despite the lack of differences between the DAILY and INT groups on fat intake, we still consider mice to have binged, as the majority of mice meet a number of previously outlined criteria. Studies that generate experimental groups based on whether rodents are prone or resistant to bingeing when first exposed to a palatable diet may serve to improve our understanding of genetic and/or mechanistic differences between these groups (Sinclair et al., 2015; Sinclair et al., 2017). Differences in ghrelin signaling via activation of its receptor may indeed play a role in binge-like intake of palatable foods, however, the results of our final studies do provide evidence of a role for GHSR in the VTA on binge-like consumption of high-fat diet under the limited access model. 


\section{Chapter 5: General Discussion}

The experiments described in this thesis utilized genetic and pharmacological approaches to test the hypothesis that the presence of GHSR is required for palatable food to drive robust appetitive and consummatory feeding behaviours. These studies were carried out in lean rodents because a number of neurophysiological alterations occur in the context of obesity, for example reduced circulating ghrelin, that could potentially confound the results. Diet induced obesity can also result in ghrelin resistance in reward processing tasks (Lockie et al., 2015; Zigman et al., 2016), the very behaviours we were interested in observing.

The data presented in Chapter 2 indicate that a lack of functional GHSRs in rats can diminish the effort expended for sucrose rewards when hungry, reduce the preference for a context previously paired with sucrose, and reduce intake of a sucrose solution when offered in conjunction with water. In the context of sucrose CPP, following the final test, we saw lower activation of the VTA in GHSR ${ }^{\mathrm{m} 1 / \mathrm{Mcwi}}$ rats, suggesting reduced motivation to obtain sucrose. Fascinatingly, rescue of the GHSR in the VTA, a region important for the wanting and liking of sweets, promotes sucrose consumption in mice that previously exhibited a reduced preference. These data highlight the importance of GHSR signaling at the level of the VTA in enhancing sucrose appetite and consumption and are consistent with previous reports of enhanced motivation for sucrose following central ghrelin administration, and intra-VTA GHSR antagonist blocking motivation for sucrose but not fasting-induced chow intake (Skibicka et al., 2011). Interestingly the antagonist effect is seen for both caloric and non-caloric sweeteners (i.e. sugar and 
saccharin; Landgren et al., 2011). The exact mechanism by which this occurs is currently unknown.

Our methodology did not allow us to specify the phenotype of neurons in which GHSRs were reinstated in the VTA. Approximately $60 \%$ of the neurons in the VTA are DAergic and approximately 30\% are GABAergic (Margolis et al., 2006; Nair-Roberts et al. 2008), thus one might expect a greater influence on DA than on GABA neurons, resulting in a direct increase in DA tone, however, there are also indirect routes through which ghrelin might influence DA tone.

The reduction in sucrose appetite and consumption associated with a decrease in GHSR signalling in the VTA, may be indicative of a role for opioidergic modulation of VTA DA neurons. Both liking and wanting of sweets are known to be mediated, in part, by opioid receptor enhancement of DA signaling (Fullerton et al., 1985; Castro and Berridge, 2014). Consistent with this, it has been shown that intra-VTA ghrelin-induced sucrose-motivated behaviour can be blocked by intra-VTA administration of naltrexone, a mu-opioid receptor (MOR) antagonist, and, moreover, that ghrelin administration elevated VTA mu-opioid receptor expression (Skibicka et al., 2012b). Thus, restoration of GHSR activity within the VTA could enhance expression of sucrose motivation and consumption by increasing MOR signaling in VTA DA neurons and conversely, a down regulation of MOR in DA neurons in GHSR ${ }^{\mathrm{m} 1 / \text { Mcwi }}$ rats could underlie their lack of sucrose motivation as well as reduced time spent in the sugar-paired chamber during CPP.

Ghrelin has also been shown to decrease GABAergic inputs onto VTA DA cells (Abizaid et al., 2006) which may occur through a MOR mediated mechanism, as opioids 
in the VTA hyperpolarize GABA interneurons (Johnson and North, 1992) and a proportion of GABA terminals are inhibited by MOR agonists (Bonci and Williams, 1997). Ghrelin receptor antagonism also reduces morphine induced CPP in rats (Jerabek et al., 2017). Therefore, if ghrelin enhances sensitivity to opioids in VTA DA neurons, whether through enhanced MOR on VTA DA cells (Skibicka et al., 2012b) or decreased GABAergic inputs (Abizaid et al., 2006) it could ultimately enhance overall DA tone, resulting in enhanced responding for sucrose rewards.

As ghrelin has been shown to potentiate CPP for a high-fat diet (Perello et al., 2010), and that GHSR antagonism in the VTA blunts preference for a diet high in fat (King et al., 2011), in Chapter 3 and 4 we tested the hypothesis that in addition to sucrose reward, GHSR activation also influences the binge eating of fat. Although the majority of rodent models that explore binge eating have been conducted in rats (e.g.s. Corwin et al., 1998; Bello et al., 2009; Boggiano et al., 2005; Hagan et al., 2002), an increase in examination of binge-like behaviours in mice has emerged in recent years (Czyzyk et al., 2010; Valdivia et al., 2014; Razzoli et al., 2015; King et al., 2016; Espinosa-Carrasco et al., 2018). The study of binge behaviours in mice aims to take advantage of the abundant transgenic technologies currently available, enabling the exploration of the molecular underpinnings of binge eating behaviours. Only two studies other than ours have examined the potential role of ghrelin in binge eating of a high-fat diet (Valdivia et al., 2014; Valdivia et al., 2015), and both demonstrate that GHSR signaling is required for the consumption of a high fat diet, as well as for the activation of the VTA following its consumption. 
In contrast to findings described in Chapter 2, the role of the GHSR in high-fat bingeing was less consistent. Initially, we were able to demonstrate that the limited access model of high fat first developed in rats (Corwin et al., 1998) was also efficacious in eliciting binge-like consumption of a high-fat diet in mice (Chapter 3, Study 2.1). In addition, mice that received the HFD intermittently, developed a binge-compensate pattern typically generated by limited intermittent access (Corwin et al., 1998; Corwin et al., 2004; Davis et al., 2007). These mice binged on fat, and subsequently ate significantly more chow than mice that received the fat daily, resulting in higher cumulative caloric intake. To investigate the role of ghrelin/GHSR signaling in the context of this model, we then exposed GHSR KO mice and their WT littermates to the limited access model (Chapter 3, Study 2.2). Results from this study suggested that the GHSR was important for high-fat bingeing, as GHSR KO mice that received fat intermittently, exhibited reduced intake of the HFD.

As in Chapter 2, we examined whether a lack of GHSR in the VTA specifically may underlie the differences between genotypes on high-fat intake, as previous findings that intra-VTA ghrelin enhances intake of a similar HFD in sated rats, and antagonizing GHSR in the VTA was able to block this effect (Wei et al., 2015), high-fat bingeing in adolescence enhanced the expression of GHSR in the VTA in adulthood (Blanco-Gandia et al., 2017), and mice lacking GHSR show reduced CPP for a HFD (Perello et al., 2010). In this latter set of experiments, we were unable to fully replicate the findings from Chapter 3 and observed no robust effects of genotype on high-fat bingeing (Study 4.1, Chapter 4). These results also did not parallel those found in the sucrose studies, in that genetic rescue of GHSR in the VTA was unable to enhance intake of fat in GHSR null 
mice. Importantly, pharmacological antagonism of GHSR in the VTA using JMV-2959 did not attenuate bingeing on fat once it had been established, suggesting that the lack of behavioural efficacy of our restoration study could not be attributed solely to the low levels of GHSR reinstatement following viral administration. Together, the data examining the role of GHSR in high-fat bingeing suggest that it does not play an integral role across all genetic models used, and we found no evidence that GHSR signalling in the VTA facilitates such behaviour. In fact, a recent study has demonstrated that in fat bingeing rats, ICV ghrelin administration enhances the intake of chow during the binge (also partially replicated after VTA administration; Bake et al., 2017), but that, akin to our last two studies, GHSR KO mice were capable of approximating the patterns of intake seen in WT mice under a binge model of access to fat.

As mentioned in the discussion of Chapter 4, it is possible that mice or rats lacking GHSR from birth develop differently and are able to acquire compensatory mechanisms that sustain homeostatic and possibly hedonic, drive to obtain food. Indeed, in most paradigms evaluating the impact of a lack of GHSR signaling on reward behaviours largely mediated by mesolimbic circuitry, following genetic KO or pharmacological inhibition of the GHSR, rats and mice still exhibit the appropriate behavioural responses but typically of reduced magnitude (Clifford et al., 2012; Wellman et al., 2012; St.Onge, 2017). Further studies are required to elucidate the potential compensatory mechanisms that may minimize behavioural differences.

It is also possible that differences inherent to the paradigms used reduce the contribution of GHSR activation. There are important distinctions between the sugar and fat studies, beyond the primary macronutrient available in each of the palatable foods 
offered. First, in many of the sucrose experiments, albeit not in the final sucrose preference study described in Chapter 2, animals were food restricted to enhance their motivation to work for and learn about cues signaling palatable food availability, as in the case of CPP. As ghrelin increases under times of FR or caloric restriction (i.e. following a fast or in a sustained negative energy balance) (Horvath et al. 2001; Wellman and Abizaid, 2015), and in response to anticipation of a scheduled meal (Cummings et al., 2001; Drazen et al., 2006; Khazall, 2017), it is likely that this elevation in ghrelin enhanced food motivated behaviours in WT rats, but not in the GHSR ${ }^{\mathrm{ml} / \mathrm{Mcwi}}$ rats, which do not respond to circulating ghrelin (MacKay et al., 2015). The bolstering of the motivational state due to FR could have contributed to the differences in behaviours between genotypes in the sucrose studies. Although, results from the two-bottle choice test would indicate that FR is not always required to observe differential responding.

As partial restoration of the GHSR in the VTA did not enhance fat intake, it is possible that the hedonically driven consumption of fat in the current model relies less heavily on the direct interaction of GHSR with DA cell bodies in the VTA, as compared to sucrose. Further, fat intake has been shown to have less of a suppressive effect on circulating ghrelin than carbohydrates (and protein) in rodents and humans (FosterSchubert et al., 2008; Rizi et al., 2018), which could result in the reduced sensitivity of our model to detect differences between GHSR KO/null WT mice on high-fat bingeing.

Using the limited access model to emulate binge eating as seen in humans was somewhat successful. In all of the binge studies, we were able to show that mice voluntarily increased their daily caloric intake as a result of exposure to HFD concurrent with standard chow, suggesting that they did like the fat. In most cases, this resulted in an 
increased net caloric intake, which may result in excessive weight gain. As the loss of control aspect of binge eating can be difficult to evaluate in animal models (Perello et al., 2014), we did not include this as a criterion for bingeing in our studies. Some investigators have highlighted the similarities between binge eating and substance dependence and suggest that the significant escalation of intake over time is analogous to tolerance seen in models of substance abuse (for review see: Corwin and Babbs, 2012). Notably, we did not see significant escalation of fat intake over time, which is often seen in these models as reported in rats (Corwin et al., 1998). This may be a function of the palatability of the food offered, but this is unlikely, as other models have used a similar diet (Corwin et al., 1998; Davis et al., 2007) and it may have more to do with how mice behave under this paradigm, as compared to rats.

The increased caloric intake of the mice following restricted access to HFD, while significant, were modest, and are likely smaller than what is reported in humans who binge eat. Moreover, models that integrate a caloric restriction and /or stress component may be more informative when it comes to translational capacity, as humans often exhibit restrained eating (Jansen et al., 1989), as well as stress-induced bingeing (Naish et al., 2018). Further, the presence of the GHSR is important for stress-induced behavioural and metabolic outcomes, including feeding on palatable foods (Patterson et al., 2010; Patterson et al., 2013; Razzoli et al., 2015; Chuang et al., 2011). Therefore, it is possible that the importance of ghrelin in binge eating would be more noteworthy if mice were challenged under a model of stress-induced binge eating. In addition, using a model of sucrose bingeing, rather than fat, may elicit greater behavioural changes and perhaps escalation of intake that was missing in our model (Avena et al., 2008, Avena et al., 
2009). Results from such studies may point to a role for VTA GHSR in sugar or high-fathigh sugar bingeing, as the combination of macronutrients has been shown to be synergistically reinforcing (DiFeliceantonio et al., 2018).

As a whole, the data indicate that signaling through the GHSR is involved in the expression of a number of food reward behaviours. As the GHSR is expressed in many regions throughout the CNS (Zigman et al., 2006), it is likely that specific feeding behaviours require coordinated action of the GHSR in areas such as the hippocampus and the lateral hypothalamus (Hsu et al., 2015), important for learning, memory, and arousal in the context of food reward. Overall, it seems that GHSR signaling generally serves to enhance the motivational state of the animal to seek, work for and consume palatable foods and this seems consistent with the notion that the ghrelin system exists to support survival of the organism in times of challenge (Mani and Zigman, 2017).

The fact that the phenotypic outcomes following manipulation of GHSR signaling are influenced heavily by paradigmatic nuances, the composition of diets offered, and the metabolic status of the animal makes manipulating GHSR activity through agonist/antagonist administration as a therapy a complex undertaking. In addition to the complexity of the system, there is another caveat to targeting the ghrelin system as an appetite-suppressant, in that, there is a body of evidence supporting a role for ghrelin in the protection from enduring psychosocial stress, which can result in anxiety and depressive-like behaviours (Lutter et al., 2008; Patterson et al., 2013). Therefore, caution should be used when attempting to target the GHSR for such purposes, as it may have unintended negative consequences. Nevertheless, basic science work evaluating the importance of activity of the ghrelin system in learning about food rewards and 
exhibiting motivation for them will help us understand some of the factors that drive palatable food overconsumption. 


\section{References}

Abizaid, A. (2009). Ghrelin and dopamine: new insights on the peripheral regulation of appetite. J Neuroendocrinol., 21(9), 787-793.

Abizaid, A. and Horvath, T.L. (2008). Brain circuits regulating energy homeostasis. Regul. Peptides, 149(1-3), 3-10.

Abizaid, A., Liu, Z.W., Andrews, Z.B., Shanborough, M., Borok, E., Elsworth, J.D., Roth, R.H., Sleeman, M.W., Piccioto, M.R. Tschop, M.H., Gao, X.B. and Horvath, T.L. (2006). Ghrelin modulates the activity and synaptic input organization of midbrain dopamine neurons while promoting appetite. Journal of Clinical Investigation, 116: 3229-3239.

Abizaid, A., Mezei, G., Thanarajasingam, G., and Horvath, T.L. (2005). Estrogen enhances light-induced activation of dorsal raphe serotonergic neurons. European Journal of Neuroscience, 21, 1536-1546.

Adan, R.A., Cone, R.D., Burbach, J.P., Gispen, W.H. (1994). Differential effects of melanocortin peptides on neural melanocortin receptors. Mol. Pharmacol., 46, 1182-1190.

Ahmed, B.Y., Chakravarthy, S., Eggers, R., Hermens, W.T., Zhang, J.Y., Niclou, S.P., Levelt, C., Sablitzky, F., Anderson, P.N., Lieberman, A.R. and Verhaagen, J. (2004). Efficient delivery of cre-recombinase to neurons in vivo and stable 
transduction of neurons using adeno-associated and lentiviral vectors. $B M C$ Neurosci, 30, 5:4.

Akamizu T. and Kangawa, K. (2011). Therapeutic applications of ghrelin to cachexia utilizing its appetite-stimulating effect. Peptides, 32(11), 2295-3000.

American Psychiatric Association (APA) (2013). Diagnostic and statistical manual of mental disorders (5th ed.). Arlington, VA: American Psychiatric Publishing. Aponte, Y., Atasoy, D., and Sternson, S.M. (2011). AGRP neurons are sufficient to orchestrate feeding behaviour rapidly and without training. Nat Neurosci., 14, 351-355.

Atalyer, D., Gibson, C., Konopacka, A. and Gelibeter, A. (2013). Ghrelin and eating disorders. Prog Neuropsychopharmacol Biol Psychiatry, 40, 70-82.

Avena, N.M., Bocarsly, M.E., Rada, P., Kim, A. and Hoebel, B.G. (2008). After daily bingeing on a sucrose solution, food deprivation induces anxiety and accumbens dopamine/acetylcholine imbalance. Physiol and Behav, 94(3), 309-315.

Avena, N.M., Rada, P. and Hoebel, B.G. (2009). Sugar and fat bingeing have notable difference in addictive-like behavior. $J$ Nutr, 139(3), 623-628.

Bahi, A., Tolle, V., Fehrentz, J.A., Brunel, L., Martinez, J., Tomasetto, C.L., Karam, S.M. (2013). Ghrelin knockout mice show decreased voluntary alcohol consumption and reduced ethanol-induced conditioned place preference. Peptides, 43, 48-55.

Bailey, K.R., Rustay, N.R. and Crawley, J.N. (2006). Behavioral phenotyping of transgenic and knockout mice: practical concerns and potential pitfalls. ILAR Journal, 47(2), 124-131. 
Bake, T., Hellgren, K. and Dickson, S.L. (2017). Acute ghrelin changes food preference from a high-fat diet to chow during binge-like eating in rodents. Journal of Neuroendocrinology, 29(4), doi.org/10.1111/jne.12463.

Bake, T., Murphy, M., Morgan, D.G.A. and Mercer, J.G. (2014). Large, binge-type meals of high fat diet change feeding behavior and entrain food anticipatory activity in mice. Appetite, $77 C, 60-71$.

Bake, T., Duncan, J.S., Morgan, D.G.A and Mercer, J.G. (2013). Arcuate nucleus homeostatic systems are not altered immediately prior to the scheduled consumption of large, binge-type meals of palatable solid of liquid diets in rats and mice. Journal of Neuroendocrinology, 25, 357-371.

Balthasar, N., Coppari, R., McMinn, J, Liu, S.M., Lee, C.E., Tang, V., Kenny, C.D., McGovern, R.A., Chua, S.C. Jr., Elmquist, J.K. and Lowell, B.B. (2004). Leptin receptor signaling in POMC neurons is required for normal body weight homeostasis. Neuron, 42(6), 983-991.

Banks, W.A., Kastin, A.J., Huang, W., Jaspan, J.B. and Maness, L.M. (1996). Leptin enters the brain by a saturable system independent of insulin. Peptides, 17, 305311.

Banks, W.A., Tschop, M., Robinson, S.M., Heiman, M.L. (2002). Extent and direction of ghrelin transport across the blood-brain barrier is determined by its unique primary structure. $J$ Pharmacol Exp Ther, 302, 822-827.

Barbano, M.F., Le Saux, M., \& Cador, M. (2009). Involvement of dopamine and opioids in the motivation to eat: influence of palatability, homeostatic state, and behavioural paradigms. Psychopharmacology. 203: 475-487. 
Bello, N.T. and Hajnal, A. (2010). Dopamine and binge eating behaviors. Pharmacology, Biochemistry and Behavior, 97(1), 25-33.

Bello, N.T., Guarda, A.S., Terrillion, C.E., Redgrave, G.W., Coughlin, J.W., and Moran, T.H. (2009). Repeated binge access to a palatable food alters feeding behavior, hormone profile, and hindbrain c-Fos responses to a test meal in adult male rats. Am J Physiol Regul Integr Comp Physiol, 297, R622-R631.

Benoit, S., Schwartz, M., Baskin, D., Woods, S.C. and Seeley, R.J. (2000). CNS melanocortin system involvement in the regulation of food intake. Horm. Behav., 37, 299-305.

Berridge, K.C., Ho, C., Richard, J.M., and DiFeliceantonio, A.G. (2010). The tempted brain eats: pleasure and desire circuits in obesity and eating disorders. Brain Resm $1350,43-64$.

Berridge, K.C. \& Robinson, T.E. (1998). What is the role of dopamine in reward: hedonic impact, reward learning or incentive salience. Brain Res. Brain Res. Review. 28(3): 309-369.

Berridge, K.C. (1996). Food reward: brain substrates of wanting and liking. Neurosci Biobehav Rev, 20(1), 1-25.

Berridge, K.C. (2009). 'Liking' and 'wanting' food rewards: brain substrates and roles in eating disorders. Physiology and Behavior, 97(5), 537-550.

Blanco-Gandia, M.C., Aracil-Fernandez, A., Montagud-Romero, S., Aguilar, M.A., Manzanares, J., Minarro, J., and Rodriguez-Arias, M. (2017). Changes in gene expression and sensitivity of cocaine reward produced by a continuous fat diet. Psychopharmacology, 234, 2337-2352. 
Blanco-Gandia, M.C., Catacorps, L., Aracil-Fernandez, A., Montagud-Romero, S., Aguillar, M.A., Manzanares, J., Valverde, O., Minarro, J. and Rodrigues-Arias, M. (2016). Effects of bingeing on fat during adolescence on the reinforcing effects of cocaine in adult male mice. Neuropharmacology, 113(a), 31-44.

Boggiano, M.M., Artiga, A.I., Pritchett, C.E., Chandler, P.C., Smith, M.L., and Eldridge, A.J. (2007). High intake of palatable food predicts binge-eating characteristics Independent of susceptibility to obesity: an animal model of lean vs. obese binge eating and obesity with and without binge eating. International Journal of Obesity, 31, 1357-67

Bonci, A. and Williams, J.T. (1997). Increased probability of GABA release during withdrawal from morphine. $J$ Neurosci, 17(2), 796-803.

Bowles, D.E., Rabinowitz, J.E. and Samulski, R.J. (2006). “The genus dependovirus,” in Parvoviruses, eds J.R. Kerr, S.F. Cottmore, M.E. Bloom., R.M. Linden, and C.R. Parrish (New York: Edward Arnold Ltd.), 15-24.

Burger, C., Gorbatyuk, O.S., Velardo, M.J., Peden, C.S., Williams, P., Zolotukhin, S. et al. (2004). Recombinant AAV viral vectors pseudotyped with viral capsids from serotypes 1,2 , and 5 display differential efficiency and cell tropism after delivery to different regions of the central nervous system. Mol. Ther, 10, 302-317.

Callahan, H.S., Cummings, D.E., Pepe, M.S., Breen, P.A., Matthys, C.C., and Weigle, D.S. (2004). Postprandial suppression of plasma ghrelin level is proportional to Ingested caloric load but does not predict inter-meal interval in humans. Journal of Clinical Endocrinology and Metabolism, 89(3), 1319-1324.

Carlin, J.L., McKee, S.E., Hill-Smith, T., Grissom, N.M., George, R., Lucki, E. and 
Reyes, T.M. (2016). Removal of high-fat diet after chronic exposure drive binge behaviour and dopaminergic dysregulation in female mice. Neuroscience, 326, 170-179.

Castro, D.C. and Berridge, K.C. (2014). Advances in the neurobiological bases for food ‘liking' vs 'wanting'. Physiology and Behavior, pii: S0031-9384(14)00299.

Cearley, C.N. and Wolfe, J.H. (2006). Transduction characteristics of adeno-associated viral vectors expressing cap serotypes 7,8,9, and Rh10 in the mouse brain. Mol. Ther., 13, 528-537.

Chen, H.Y., Trumbauer, M.E., Chen, A.S., Weingarth, D.T., Adams, J.R., Frazier, E.G. et al. (2004). Orexigenic action of peripheral ghrelin is mediated by neuropeptide y and agouti-related protein. Endocrinology, 145, 2607-2612.

Chuang, J.C., Perello, M., Sakata, I., Osborne-Lawrence, S., Savitt, J.M., Lutter, M., and Zigman, J.M. (2011) Ghrelin mediates stress-induced food-reward behavior in mice. JCI, 121(7), 2684-92.

Clifford, P.S., Rodriquez, J., Schul, D., Hughes, S., Kniffin, T., Hart, N., Eitan, S., Brunel, L., Fehrentz, J.A., Martinez, J., and Wellman, P.J. (2011). Attenuation of cocaine-induced locomotor sensitization in rats sustaining genetic or pharmacologic antagonism of ghrelin receptors. Addict Biol, 17(6), 956-963.

Cone, R.D., Cowley, M.A., Butler, A.A., Fan, W., Marks, D.L. and Low, M.J. (2001). The arcuate nucleus as a conduit for diverse signals relevant to energy homeostasis. Int J Obes Relat Metab Disord., 25, S63-70.

Cone, R.D. (2005). Anatomy and regulation of the central melanocortin system. Nature Neuroscience, 8, 571-578. 
Coons, E.E. \& Cruce, J.A. (1968). Lateral hypothalamus: food current intensity in maintaining self-stimulation of hunger. Science. 159(918): 1117-1119.

Corwin, R.L. and Babbs, K. (2012). Rodent models of binge eating: are they models of addiction? ILAR Journal, 53(1), 23-34.

Corwin, R.L. (2004). Binge-type eating induced by limited access in rats does not require energy restriction on the previous day. 42(2), 139-142.

Corwin, R.L. and Hajnal, A. (2005). Too much of a good thing: neurobiology of nonhomeostatic eating and drug abuse. Physiology and Behavior, 86, 5-8.

Corwin, R.L., Wojnicki, F.H.E., Fisher, J.O., Dimitriou S.G., Rice, H.B., and Young, M.A. (1998). Limited access to a dietary fat option affects ingestive behavior but not body composition in male rats. Physiology and Behavior, 63(3), 543-553.

Cowley, M.A., Smith, R.G., Diano, S., Tschop, M., Pronchuck, N., Grove, K.L. et al. (2003). The distribution and mechanism of action of ghrelin in the CNS demonstrates a novel hypothalamic circuit regulating energy homeostasis. Neuron, 37, 649-661.

Cummings, D.E. (2006). Ghrelin and the short- and long-term regulation of appetite and body weight. Physiol and Behav,89(1), 71-84.

Cummings, D.E., Purnell, J.Q., Frayo, R.S., Schmidova, K., Wisse, B.E., and Weigle, D.S. (2001). A preprandial rise in plasma ghrelin levels suggests a role in meal initiation in humans. Diabetes, 50, 1714-1719.

Cummings, D.E., Frayo, R.S., Marmonier, C., Aubert, R. and Chapelot, D. (2004). 
Plasma ghrelin levels and hunger scores in humans initiating meals voluntarily without time-and food-related cues. Am J Physiol Endocrinol Metab, 287(2), 297304.

Czyzyk, T.A., Sahr, A.E. and Statnick, M.A. (2010). A model of binge-like eating behavior that does not require food deprivation or stress. Obesity, 18(9), 17101717.

da Luz, F.Q., Hay, P., Touyz, S. and Sainsbury, A. (2018). Obesity with comorbid eating disorders: associated health risks and treatment approaches. Nutrients, 10, 829, 19.

Date, Y., Kojima, M., Hosoda, H., Sawaguchi, A., Mondal, M.S., Suganuma, T., Matsukura, S., Kangawa, and Nakazato, M. (2000). Ghrelin, a novel growth hormone-releasing acylated peptide, is synthesized in a distinct endocrine cell type in the gastrointestinal tracts of rats and humans. Endocrinology, 141, 42554261.

Davis, C.A., Levitan, R.D., Reid, C., Carter, J.C., Kaplan, A.S., Patte, K.A., King, N., Curtis, C. and Kennedy, J.L. (2009). Dopamine for "wanting" and opioids for "liking": a comparison of obese adults with and without binge eating. Obesity, 17(6), 1220-1225.

Davis, J.F., Melhorn, S.J., Shurdak, J.D., Heiman, J.U., Tschop, M.H., Clegg, D.J. and Benoit, S.C. (2007). Comparison of hydrogenated vegetable shortening and nutritionally complete high-fat diet on limited access-binge behavior in rats. Physiology and Behavior, 92, 924-930

Davis, K.W., Wellman, P.J. and Clifford, P.S. (2007). Augmented cocaine conditioned 
place preference in rats pretreated with systemic ghrelin. Regul Pept, 140(3), 148152.

Day, J.J., Roitman, M.F., Wightman, R.M. and Carelli, R.M. (2007). Associative learning mediates dynamic shifts in DA signaling in the nucleus accubens. Nat Neuroscim 10(8), 1020-1028.

DeBoer, M.D. (2011). Ghrelin and cachexia: will treatment with GHSR-1a agonists make a difference for patients suffering from chronic wasting syndromes? Molecular and Cellular Endocrinology, 1(20), 97-105.

Denis, R.G., Joly-Amado, A., Domingos, A.I., Vaynshteyn, J., Voss, H.U., Ren, X., Gradinaru, V., Zang, F., Deisseroth, K., deAraujo, I.E., and Freidman, J. (2011). Leptin regulates the reward value of nutrient. Nature Neuroscience, 14(12), 15621568.

Dickson, S.L., Egecioglu, E., Landgren, S., Skibicka, K.P., Engel, J.A. and Jerlhag, E. (2011). The role of the central ghrelin system in reward from food and chemical drugs. Molecular and Cellular Endocrinology, 340: 80-87.

Dimitrou, S.G., Rice, H.B. and Corwin, R.L. (2000). Effects of limited access to a fat option on food intake and body composition in female rats. Int J Eat Disrod., 28(4), 436-445.

Drazen, D.L., Vahl, T.P., D’Alessio, D.A., Seeley, R.J. and Woods, S.C. (2006). Effects of a fixed meal pattern on ghrelin secretion: evidence for a learned response independent of nutrient status. Endocrinology, 147(1), 23-30.

Drewnowski, A., Shrager, E.E., Lipsky, C., Stellar, E., and Greenwood, M.R. (1989). 
Sugar and fat: sensory and hedonic evaluation of liquid and solid foods. Physiol Behav., 45, 177-183.

Druce, M.R., Wren, A.M., Park, A.J., Milton, J.E., Patterson, M., Frost, G., et al. (2005). Ghrelin increases food intake in obese as well as lean subjects. Int $J$ Obes (London), 29, 1130-1136.

Elias, C.F., Aschkenasi, C., Lee, C., Kelly, J., Ahima, R.S., Bjorbaek, C., Flier, J.S., Saper, C.B. and Elmqist, J.K. (1999). Leptin differentially regulates NPY and POMC neurons projecting to the lateral hypothalamic area. Neuron, 23(4), 775786.

Elias, C.F., Kelly, J.F., and Lee, C.E., Ahima, R.S., Drucker, D.J., Saper, C.B. and Elmquist, J.K. (2000). Chemical characterization of leptin-activated neurons in the rat brain. J Comp Neurol., 423(2), 261-281.

Elmore DK and di Castro JM. (1991). Meal patterns of normal, untreated bulimia nervosa and recovered bulimic women. Physiology and Behavior, 49, 99-105.

Elmquist, J.K., Elias, C.F., \& Saper, C.B. (1999). From lesions to leptin: hypothalamic control of food intake and bodyweight. Neuron. 22: 221-232.

Elmquist, J.K., Bjorbaek, C., Ahima, R.S., Flier, J.S. and Saper, C.B. (1998).

Distributions of leptin receptor mRNA isoforms in the rat brain. $J$ Compar Neurol., 395, 535-547.

Espinosa-Carrasco, J., Burokas, A., Fructuoso, M., Erb, I., Martin-Garcia, E., GutierrezMartos, M., Notredame, C., Maldonado, R. and Dierssen, M. (2018). Time-course and dynamics of obesity-related behavioral changed induced by energy-dense foods in mice. Addict Biol, 23, 531-543. 
Everitt, B.J. \& Robbins, T.W. (2005). Neural systems of reinforcement for drug addiction: from actions to habits to compulsion. Nature Neuroscience. 8(11),1481-1489.

Faulconbridge, L.F., Cummings, D.E., Kaplan, J.M., \& Grill, H.J. (2003). Hyperphagic effects of brainstem ghrelin administration. Diabetes. 52: 2260- 2265.

Fernandez, G., Cabral., A., Cornejo, M.P., De Francesco, P.N., Garcia-Romero, G., Reynaldo, M. and Perello, M. (2016). Des-acyl ghrelin directly targets the arcuate nucleus in a ghrelin-receptor independent manner and impairs the orexigenic effect of ghrelin. J Neuroendorcinol, 28(2), 12349.

Figlewicz, D.P., Higgins, M.S., Ng-Evans, S.B and Havel, P.J. (2001). Leptin reverses sucrose-conditioned place preference in food-restricted rats. Physiology and Behavior, 73, 229-234.

Figlewicz, D.P. and Sipols, A.J. (2010). Energy regulatory signals and food reward. Pharmacol. Biochem, Behav., 97, 15-24.

Foster-Shubert, K.E., Overduin, J., Prudom, C.E., Liu, J., Callahan, H.S., Gaylinn, B.D., Thorner, M.O. and Cummings, D.E. (2008). Acyl and total ghrelin are suppressed strongly by ingested proteins, weakly by lipids, and biphasically by carbohydrates. J Clin Endocrinol Metab, 93(5), 1971-1979.

Frazao, R., Dungan Lemko, H.M., DaSilva, R.P., Ratra, D.C., Lee, C.E., Williams, K.W., Zigman, J.M. and Elias, C.F. (2014). Estradiol modulates kiss1 neuronal response to ghrelin. Am J Physiol Endocrinol Metab, 306, e606-e614.

Fullerton, D.T., Getto, C.J., Swift, W.J. and Carlson, I.H. (1985). Sugar, opioids and binge eating. Brain Research Bulletin, 14, 673-680. 
Fulton, S., Woodside, B., Shizgal, P. (2000). Modulation of brain reward circuitry by leptin. Science, $317,125-128$.

Geliebter, A., Hashim, S.A. and Gluck, M.E. (2008). Appetite-related gut peptides, ghrelin, PYY, and GLP-1 in obese women with and without binge eating disorder. Physiol Behav, 94(5), 696-699.

Geliebter, A., Gluck, M.E. and Hashim, S.A. (2005). Plasma ghrelin concentrations are lower in binge-eating disorder. J Nutr, 135(5), 1326-1330.

Glover, C.P.J., Bienemann, A.S., Heywood, D.J., Cosgrave, A.S. and Uney, J.B. (2002). Adenovrial-mediated, high-level, cell-specific transgene expression: a syn1-wpre cassette mediates increased transgene expression with no loss of neuron specificity. Mol Ther, 5(5), 509-516.

Gnanapavan, S., Kola, B, Bustin, S.A., Morris, D.G., McGee, P., Fairclough, P. et al., (2002). The tissue distribution of the mRNA of ghrelin and subtypes of its receptor, GHS-R, in humans. J Clin Endo Metab, 87, 2988.

Gold, R.M. (1973). Hypothalamic obesity: the myth of the ventromedial nucleus. Science, 182, 488-490.

Guan, X.M., Yu, H., Palyha, O.C., McKee, K.K., Feighner, S.D., Sirinathsinghji, D.J.S., Smith, R.G., Van der Ploeg, L.H.T. and Howard, A.D. (1997). Distribution of mRNA encoding the growth hormone secretagogue receptor in brain and peripheral tissues. Molecular Brain Research, 48, 23-29.

Gutierrez, J.A., Solenberg, P.J., Perkins, D.R., Willency, J.A., Knierman, M.D., Jin, Z. et al. (2008). Ghrelin octanoylation mediated by an orphan lipid-transferase. Proc Natl Acad Sci USA, 105, 6320-6325. 
Hagan, M.M., Wauford, P.K., Chandler, P.C., Jarrett, L.A., Rybak, R.J., and Blackburn, K. (2002). A new animal model of binge eating: key synergistic role of past caloric restriction and stress. Physiol Behav, 77(1), 45-54.

Halpern, C.H., Tekriwal, A., Santollo, J., Keating, J.G., Wolf, J.A., Daniels, D. and Bale, T.L. (2013). Amelioration of binge eating by nucleus accumbens deep brain stimulation in mice involves D2R modulation. Journal of Neuroscience, 33(17), $7122-7129$.

Harris, R.B.S. \& Martin, R.J. (1984). Recovery of body weight from below "set point" in mature female rats. The Journal of Nutrition. 114: 1143-1150.

Hentges, S.T., Nishiyama, M., Overstreet, L.S., Stenzel-Poore, M., Williams, J.T. and Low, M.J. (2004). GABA release from proopiomelanocortin neurons. $J$ Neuroscience, 24(7), 1578-1583.

Hetherington, A.W. (1941). The relation of various hypothalamic lesions to adiposity and other phenomena in the rat. Amer. J. Physiol., 133, 326-327.

Hetherington, A.W. \& Ranson, S.W. (1942). The spontaneous activity and food intake of rats with hypothalamic lesions. Am. J. Physiol. 136, 609-617.

Hodos, W. (1961). Progressive ratio as a measure of reward strength. Science, 134(3483), 943-4.

Hoebel, B.G. (1997). Neuroscience and appetitive behaviour research: 25 years. Appetite, 29(2), 119-133.

Holst, B., Cygankiewiczm, A., Jensen, T.H., Andersen, M. and Schwartz, T.W. (2003). High constitutive signaling of the ghrelin receptor-identification of a potent inverse agonist. Molecular Endocrinology, 17(11), 2201-2210. 
Horvath, T.L., Bechmann, I., Naftolin, F., Kalra, S.P. and Leranth, C. (1997).

Heterogeneity in the neuropeptide y-containing neurons of the rat arcute nucleus: GABAergic and non-GABAergic subpopulations. Brain Res, 755(1-2), 283-286.

Horvath, T.L., Diano, S., Sotonyi, P., Heiman, M. and Tschop, M. (2001). Minireview: ghrelin and the regulation of energy balance-a hypothalamic perspective. Endocrinology, 142: 4163-4169.

Hosoda, H., Kojima, M. \& Kangawa, K. (2006). Biological, physiological and pharmacological aspects of ghrelin. J Pharmacol. Sci. 100: 398-410.

Hotta, M., Ohwada, R., Akamizu, T., Shibasaki, T., Takano, K., Kangawa, K. (2009). Ghrelin increases hunger and food intake in patients with restricting-type anorexia nervosa: a pilot study. Endocrine Journal, 56, 1119-128.

Howard, A.D. et al. (1996). A receptor in pituitary and hypothalamus that functions in growth hormone release. Science, 273, 974-977.

Hsu, T., Hahn, J., Konavur, V., Noble, E., Suarez, A., Thai, J., Nakamoto, E. and Kanoski, S. (2015). Hippocampus ghrelin signaling mediates appetite through lateral hypothalamic orexin pathways. eLife, doi: 10.755/eLife.11190.001.

Hudson, J.I., Hiripi, E., Pope Jr., H.G., and Kessler, R.C. (2007). The prevalence and correlates of eating disorders in the national comorbidity survey replication. Biological Psychiatry, 61(3), 348-358.

Inhoff, T., Monikes, H., Noetzel, S., Stengel, A., Goebel, M., Dinh, Q.T. et al. (2008). Desacyl ghrelin inhibits the orexigenic effect of peripherally injected ghrelin in rats. Peptides, 29, 2159-2168.

Jansen, A., Klaver, J., Merckelbach, M., and van den Hout (1989). Restrained eaters are 
rapidly habituating sensation seekers. Behaviour Research and Therapy, 27, 247252.

Jerabek, P., Havlickova, T., Pushkina, N., Charalambous, C., Lapka, M., Kacer, P. and Sustkova-Fiserova, M. (2017). Ghrelin receptor antagonism of morphine-induced conditioned place preference and behavioural and accumbens dopaminergic sensitization in rats. Neurochemistry International, DOI:

10.1016/j.neuint.2017.09.013.

Jerlhag, E., Egecioglu, E., Dickson, S.L., Andersson, M., Svensson, L., and Engel, J.A. (2006). Ghrelin stimulates locomotor activity and accumbal dopamine overflow via central cholinergic mechanisms: implications for its involvement in brain reward. Addiction Biology, 11:45-54.

Jerlhag, E., Egecioglu, E., Dickson, S.L., Douhan, A., Svensson, L. and Engel, J.A. (2007). Ghrelin administration stimulates locomotor activity and increases extracellular concentration of dopamine in the nucleus accumbens. Addiction Biology, 12:6-16.

Jerlhag. E. (2008). Systemic administration of ghrelin induces conditioned place preference and stimulates accumbal dopamine. Addiction Biology, 13, 358-363.

Johnson, S.W. and North, R.A. (1992). Opioids excite dopamine neurons by hyperpolarization of local interneurons. $J$ Neurosci, 12(2), 483-488.

Johnson, A.W., Canter, R., Gallagher, M. and Holland, P. (2009). Assessing the role of the growth hormone secretagogue receptor in motivational learning and food intake. Behavioural Neuroscience, 123(5), 1058-1065.

Johnson, P. M., \& Kenny, P. J. (2010). Dopamine D2 receptors in addiction-like 
reward dysfunction and compulsive eating in obese rats. Nature Neuroscience, 13(5), 635-641.

Kales, E.F. (1990). Macronutrient analysis of binge eating in bulimia. Physiology and Behavior, 48(6), 837-840.

Kelley, A.E. \& Berridge, K.C. (2002). The neuroscience of natural rewards: relevance to addictive drugs. The Journal of Neuroscience. 22(9), 3306-3311.

Kelley, A.E., Will, M.J., Steininger, T.L., Zhang, M., and Haber, S.N. (2003). Restricted daily consumption of a highly palatable food (chocolate Ensure $\left.{ }^{\circledR}\right)$ alters striatal enkephalin gene expression. Eur J Neurosci, 18, 2592-2598.

Kelley, A.E. (2004). Ventral striatal control of appetitive motivation: role in ingestive behavior and reward-related learning. Neuroscience and Biobehavioral Reviews, 27, 765-776.

Kessler, R.C. Berglund, P.A., Chiu, W.T., Deitz, A.C., Hudson, J.I., Shahly, V., AguillarGaxiola, S., Alonso, J., Angermeyer, M.C., Benjet, C., Bruffaerts, R., deGirolamo, G., deGraaf, R., Haro, J.M., Kovess-Masfety, V., O’Neill, S., Posada-Villa, J., Sasu, C., Scott, K., Viana, M.C. and Xavier, M. (2013). The prevalence and correlates of binge eating disorder in the world health organization world mental health surveys. Biological Psychiatry, 73, 904-914.

Khazall, R. (2017). The food entrainable oscillator: the ghrelin and growth hormone secretagogue story. (Dissertation).

Kopelmann, P.G. (2000). Obesity as a medical problem. Nature, 404 (6778), 635-43.

Kojima, M., Hosoda, H., Date, Y., Nakazato, M., Matsuo, H., Kangawa, K. (1999). 
Ghrelin is a growth-hormone releasing acylated peptide from stomach. Nature, 402, 656-660.

Kojima, M. and Kangawa, K. (2005). Ghrelin: structure and function. Physiol Rev, 85, 495-522.

Kreisler, A.D., Garcia, M.G., Spierling, S.R., Hui, B.E., and Zorilla, E.P. (2017). Extended vs. brief/intermittent access to palatable food differently promote binge-like intake, rejection of less preferred food, and weight cycling in female rats. Physiol Behav, 177, 305-316.

Landgren, S., Simms, J.A., Thelle, D.S., Strandhagen, E., Bartlett, S.E., Engel, J.A. and Jerlhag, E. (2011). The ghrelin signaling system is involved in the consumption of sweets. PLoS One, 6(3), e18170.

Lardeux, S., Kim, J.J., and Nicola, S.M. (2013). Intermittent access to sweet high-fat liquid induces increased palatability and motivation to consume in a rat model of binge consumption. Physiology and Behavior, 114-115, 21-31.

Lawrence, C.B., Snape, A.C., Baudoin, F.M. and Luckman, S.M. (2002). Acute central ghrelin and GH secretagogues induce feeding and activate brain appetite centres. Endocrinology, 143(1), 155-162.

Lawrence, V.J. and Kopelman, P.G. (2004). Medical consequences of obesity. Clinical Dermatology, 22(4), 296-302.

Leibowitz, S.F., Hoebel, B.G. Behavioural Neuroscience and obesity. In: Bray, G., Bouchard, C., James, P. (eds.). The Handbook of Obesity. New York: Marcel Dekker; (2004) P.301-371.

Lindqvist, A., de la Cour, C.D., Stegmark, A., Hakanson, R. and Erlanson-Albertsson, C. 
(2005). Overeating of palatable food is associated with blunted leptin and ghrelin responses. Regulatory Peptides, 130, 123-132.

Liu, S., Globa, A.K., Mills, F., Naef, L., Qiao, M., Bamji, S.X. and Borgland, S.L. (2016). Consumption of palatable food primes food approach behaviour by rapidly increasing synaptic density in the VTA. PNAS, 113,9, 2520-2525.

Liu, S. and Borgland, S.L. (2015). Regulation of the mesolimbic dopamine circuit by feeding peptides. Neuroscience, 289, 19-42.

Lockie, S.H., Dinan, T., Lawrence, S.J. and Andrews, Z.B. (2015). Diet-induced obesity causes ghrelin resistance in reward processing tasks. Psychoneuroendocrinology, $62,114-120$.

Lutter, M., Sakata, I., Osborne-Lawrence, S., Rovinsky, S.A., Anderson, J.G., Jung, S., Birnbaum, S., Yanagisawa, M., Elmquist, J.K., Nestler, E.J. and Zigman, J.M. (2008). The orexigenic hormone ghrelin defends against depressive symptoms of chronic stress. Nat Neurosci, 11(7), 752-3.

MacKay, H., Charbonneau, V.R., St-Onge, V., Murray, E., Watts, A., Wellman, M.K. and Abizaid, (2016). Rats with a truncated ghrelin receptor (GHSR) do not respond to ghrelin, and show reduced intake of a palatable, high-calorie food. Physiol Behav, 163, 88-96.

Malik, S., McGlone, F., Bedrossian, D. \& Dagher, A. (2008). Ghrelin modulates brain activity in areas that control appetitive behavior. Cell Metabolism. 7(5), 400-409.

Mani, B.K. and Zigman, J.M. (2017). Ghrelin as a survival hormone. Trends in Endocrinology and Metabolism, 28(12), 843-854.

Margolis, E.B., Lock, H., Hjelmstad, G.O. et al. (2006). The ventral tegmental area 
revisited: is there an electrophysiological marker for dopaminergic neurons? $J$ Physiol, 577, 907-924.

Martin-Garcia, E., Fernandez-Castillo, N., Burokas, A., Gutierrez-Cuesta, J., SanchezMora, C., Casas, M., Ribases, M, Cormand, B., and Maldonado, R. (2014). Frustrated expected reward induces differential transcriptional changes in the mouse brain. Addition Biology, 20, 22-37.

Meye, F.J. and Adan, R.A.H. (2014). Feelings about food: the ventral tegmental area in food reward and emotional eating. Trends in Pharm Sci, 35(1), 31-40.

Michaelides, M, Thanos, P.K., Volkow, N.D., and Wang, G.J. (2012). Dopamine-related fronto-striatal abnormalities in obesity and binge-eating disorder: emerging evidence for developmental psychopathology. Int Rev Psychiatry, 24, 21-218.

Monteleone, P., Tortorella, A., Castaldo, E., Di Filippo, C. and Maj, M. (2007). The Leu72Met polymorphism of the ghrelin gene is significantly associated with binge eating disorder. Psychiatr Genet, 17(1), 13-16.

Monteleone, P., Tortorella, A., Castaldo, E., Di Fillippo, C. and Maj, M. (2006). No association of the Arg51Gln and Leu72Met polymorphisms of the ghrelin gene with anorexia nervosa or bulimia nervosa. Neurosci Lett, 398(3), 325-327.

Monteleone, P., Fabrazzo, M., Tortorella, A., Martiadis, V., Serritella, C. and Maj, M. (2005). Circulating ghrelin is decreased in non-obese and obese women with binge eating disorder as well as in obese non-binge eating women, but not in patients with bulimia nervosa. Psychoneuroendocrinology, 30(3), 243-250.

Morales, M. and Margolis, E.B. (2017). Ventral tegmental area: cellular heterogeneity, connectivity and behaviour. Nature Reviews Neuroscience, 18, 73-85. 
Morton, G.J., Meek, T.H., and Schwartz, M.W. (2014). Neurobiology of food intake in health and disease. Nature Reviews Neuroscience, 15, 367-378.

Murlidharan, G., Samulski, R.J. and Asokan, A. (2014). Biology of adeno-associated viral vectors in the central nervous system. Frontiers in Molecular Neuroscience, 7,76 .

Nagaya, N., Uematsu, M., Kojima, M., Date, Y., Nakazato, M., Okumura, H., Hosoda, H., Shimizu, W., Yamagishi, M., Oya, H., Yutani, C., \& Kangawa, K. (2001). Elevating circulating levels of ghrelin in cachexia associated with chronic heart failure. Circulation, 104: 2034-2038.

Nair-Roberts, R.G., Chatelain-Badie, S.D., Benson, E. et al. Stereological estimates of dopaminergic, GABAergic and glutamatergic neurons in the ventral tegmental area, substantia nigra, and retrorubral field in the rat. Neuroscience, 152, 10241031.

Nakazato, M., Murakami, N., Date, Y., Kojima, M., Matsuo, H., Kangawa, K. and Matsukura, S. (2001). A role for ghrelin in the central regulation of feeding. Nature, 409, 194-198.

Naish, K.R., Laliberte, M., MacKillop, J. and Balodis, I.M. (2018). Systematic review of acute stress in binge eating disorder. European Journal of Neuroscience, doi: 10.1111/ejn.14110.

Naleid, A.M., Grace, M.K., Cummings, D.E., \& Levine, A.S. (2005). Ghrelin induces feeding in the mesolimbic reward pathway between the ventral tegmental area and the nucleus accumbens. Peptides, 26, 2274-2279.

Norgren et al., (2006). Gustatory reward and the nucleus accumbens. Phsyiol. Behav., 89, 
$531-535$.

Overduin, J., Figlewicz, D.P., Bennett-Jay, J., Kittleson, S., and Cummings, D.E. (2012). Ghrelin increases the motivation to eat but does not alter food palatability. Am J Physiol Integr Physioli, 303, R259-R69.

Overduin, J., Frayo, R.S., Grill, H.J., Kaplan, J.M., and Cummings, D.E. (2005). Role of the duodenum and macronutrient type in ghrelin regulation. Endocrinology, $146(2), 845-850$.

Parylak, S.L., Cottone, P., Sabino, V., Rice, K.C., and Zorilla, E.P. (2008). Effects of CB1 and CRF1 receptor antagonists on binge-like eating in rats with limited access to a sweet-fat diet: lack of withdrawal-like responses. Physiol \& Behav, 107(2), 231-242.

Patterson, Z.R., Khazall, R., Mackay, H., Anisman, H. and Abizaid, A. (2013). Central ghrelin signaling mediates the metabolic response of C57BL/6 male mice to chronic social defeat stress. Endocrinology, 154(3), 1080-1091.

Patterson, Z.R., Ducharme, R., Anisman, H., and Abizaid, A. (2010). Altered metabolic and neurochemical responses to chronic unpredictable stressors in ghrelin receptor deficient mice. European Journal of Neuroscience, 32(4), 632-639.

Pelchat, M.L., Johnson, A., Chan, R., Valdez, J., and Ragland, J.D. (2004). Images and desire: food-craving activation during fMRI. Neuroimage, 23, 1486-1493.

Perello, M., Validvia, S., Garcia Romero, G and Raingo, J. (2014). Considerations about rodent models of binge eating episodes. Front Psychol., 29(5), 372.

Perello M. and Zigman, J.M (2012). The role of ghrelin in reward-based eating. Biol Psychiatry, 72, 347-353. 
Perello, M., Sakata, I., Birnbaum, S., Chuang, J., Osborne-Lawrence, S., Rovinsky, S.A., Woloszyn, J., Yanagisawa, M., Lutter, M. and Zigman, J.M. (2010). Ghrelin increases the rewarding value of a high-fat diet in an orexin-dependent manner. Biological Psychiatry, 67, 880-886.

Pinel, J.P. and Huang, E. (1976). Effects of periodic withdrawal on ethanol and saccharin selection in rats. Physio Behav, 16(6), 693-698.

Pothos, E.N. and Sulzer, D. (1998) Plasticity of quantal size in ventral midbrain dopamine neurons: possible implications for the neurochemistry of feeding and reward. Appetite, 31, 405.

Prieto-Garcia, Egecioglu, E., Studer, E., Westberg, L. and Jerlhag, E. (2015). Ghrelin and GHS-R1a signaling withing the ventral and laterodorsal tegmental area regulate sexual behaviour in sexually naïve male mice. Psychoneuroendocrinology, 62, $392-402$.

Quarta, D., DiFrancesco, C., Melotto, S., Mangiarini, L., Heidbreder, C., \& Hedou, G. (2009). Systemic administration of ghrelin increases extracellular dopamine in the shell but not the core of the nucleus accumbens. Neurochemistry International. 54: 89-94.

Razzoli, M., Sanghez, V., and Bartolomucci A. (2015). Chronic subordination stress induces hyperphagia and disrupts eating behaviour in mice modelling bingeeating-like disorder. Front Nutr, 1(30), pii: 00030.

Rizi, E.P., Loh, T.P., Baig, S., Chhay, V., Huang, S., Quek, J.C., Tai, E.S., Toh, S. and 
Khoo, C.M. (2018). A high carbohydrate, but not fat or protein meal attenuates postprandial ghrelin, PYY and GLP-1 responses in Chinese men. PLoS One, doi.org/10.1371/journal.pone.0191609.

Rotiman, M.F., Stuber, G.D., Phillips, P.E.M., Wightman, R.M., and Carelli, R.M. (2004). Dopamine operates as a subsecond modulator of food seeking. J Neurosci, 24(6), 1265-1271.

Salamone, J.D. \& Correa, M. (2002). Motivational views of reinforcement: implications for understanding the behavioral functions of nucleus accumbens dopamine. Behav. Brain Research. 137, 3-25.

Salamone, J.D., Steinpreis, R.E., McCullough, L.D., Smith, P., Grebel, D., \& Mahan, K. (1991). Haloperidol and nucleus accumbens dopamine depletion suppress lever pressing for food but increase free food consumption in a novel food choice procedure. Psychopharmacology. 104: 515-521.

Samulski, R.J. and Muzyczka, N. (2014). AAV-mediated gene therapy for research and therapeutic purposes. Anny. Rev. Virol., 1, 427-451.

Saper, C.B., Chou, T.C., and Elmquist, J.K. (2002). The need to feed: homeostatic and hedonic control of eating. Neuron, 36, 199-211.

Satou, M., Nakamura, Y., Ando, H. and Sugimoto, H. (2011). Understanding the functional significance of ghrelin processing and degradation. Peptides, 32, 21832190.

Schaeffer, M., Langlet, F., Lafont, C., Molino, F., Hodson, D.J., Roux, T., Lamarque, L., Verdie, P., Bournier, E., Dehouck, B., Baneres, J-L., Martinez, J., Mery, P-F., Marie, J., Trinquet, E., Fehrentz, J-A., Prevot, V., and Mollard, P. (2013). Rapid 
sensing of circulating ghrelin by hypothalamus appetite-modifying neurons. PNAS, 110(4), 1512-1517.

Schultz, W. (1998). Predictive reward signal of dopamine neurons. Journal of Neurophysiology. 80: 1-27.

Schwartz, M.W., Woods, S.C., Porte Jr., D., Seeley, R.J. and Baskin, D.G. (2001). C Central nervous system control of food intake. Nature, 404, 661-671.

Schwartz, M.W., Woods, S.C., Seeley, R.J., Barsh, G.S., Baskin, D.G., and Leibel, R.L. (2003). Is the energy homeostasis system inherently biased towards weight gain? Diabetes, 52, 232-238.

Schwartz, G.J. and Zeltser, L.M. (2013). Functional organization of neuronal and humoral signals regulating feeding behavior. Annu Rev Nutr, 33, 1-21.

Schmid, D.A., Heid, K., Ising, M., Uhr, M., Weikel, J.C. and Steiger, A. (2005). Ghrelin stimulates appetite, imagination of food, $\mathrm{GH}, \mathrm{ACTH}$, and cortisol, but does not affect leptin in normal controls. Neuropsychopharmacology, 30, 1187-1192.

Sclafani, A. (2000). Oral and postoral determinants of food reward. Physiol Behav, 72(6), 773-779.

Shanmugarajah, L., Dunigan, A.I., Frantz, K.J. and Roseberry, A.G. (2017). Altered sucrose self-administration following injection of melanocortin receptos agonists and antagonists into the ventral tegmental area. Psychopharmacology, 234(11), 1682-1692.

Sharma, S., Hryhorczuk, C, and Fulton, S. (2012). Progressive-ratio responding for palatable high-fat diet and high-sugar food in mice. J Vis Exp., 63, doi: $10.3791 / 3754$. 
Sharpe, M.J., Clemens, K.J., Morris, M.J., ad Westbrook, R.F. (2016). Daily exposure to sucrose impairs subsequent learning about food cues: a role for alterations in ghrelin signaling and dopamine $\mathrm{d} 2$ receptors. Neuropsychopharmacology, 41, $1357-1365$.

Shimbara, T., Mondal, M.S., Kawagoe, T., Toshinai, K., Koda, S., Yamaguchi, H., Date, Y., and Nakazato, M. (2004). Central administration of ghrelin preferentially enhances fat ingestion. Neurosci Lett, 369(1), 75-79.

Sinclair, E.B., Hildebrandt, B.A., Culbert, K.M., Klump, K.L. and Sisk, C.L. (2017). Preliminary evidence of sex differences in behavioural and neural responses to palatable food in rodents. Physiology and Behaviour, 176, 165-173.

Sinclair, E.B., Culbert, K.M., Gradl, D.R., Richardson, K.A., Klump, K.L. and Sisk, C.L. (2015). Differential mesocorticolimbic responses to palatable food in binge eating prone and binge eating resistant female rats. Physiology and Behaviour, 152, 249256.

Skibikca, K.P., Hansson, C., Egecioglu, E. and Dickson, S.L. (2012a). Role of ghrelin in food reward: impact of ghrelin on sucrose self-administration and mesolimbic dopamine and acetylcholine receptor gene expression. Addict Biol, 17(1), 95-107.

Skibicka, K.P., Shirazi, R.H., Hansson, C. and Dickson, S.L. (2012b). Ghrelin interacts with neuropeptide Y Y1 and opioid receptors to increase food reward. Endocrinology, 153(3), 1194-1205.

Skibicka, K.P., Hansson, C., Alvarez-Crespo, M., Friberg, P.A. and Dickson, S.L. (2011a). Ghrelin directly targets the ventral tegmental area to increase food motivation. Neuroscience, 180, 129-137. 
Skibicka, K.P. and Dickson, S.L. (2011b). Ghrelin and food reward: the story of potential underlying substrates. Peptides, 32, 2265-2273.

Smink, F.R., vanHoeken, S., and Hoek, H.W. (2012). Epidemiology of eating disorders: incidence, prevalence and mortality rates. Curr Psychiatry Rep, 14, 406-414. Smith, K.L., Rao, R.R., Velazquez-Sanchez, C., Valenza, M., Giuliano, C., Everitt, B.J., Sabino, V., and Cottone, P. (2014). The uncompetitive N-methyl-Daspartate antagonist memantine reduces binge like eating, food-seeking behavior, and compulsive eating: role of the nucleus accumbens shell. Neuropsychopharmacology, 1-9.

Spiegelman, B.M. and Flier, J.S. (2001). Obesity and the regulation of energy balance. Cell, 104, 531-543.

Spyraki,C., Fibiger, H.C., and Phillips, A.G. (1982). Attenuation by haloperidol of place preference conditioning using food reinforcement. Psychopharmacology, 77(4), $379-382$.

Squire, L.R., Berg, D., Bloom F.E., duLac, S., Ghosh, A., \& Spitzer, N.C. (Eds.). (2008). Fundamental Neuroscience ( $3^{\text {rd }}$ ed.). California: Elsevier.

Stark, R., Santos, V.V., Geenen, B., Cabral, A., Dinan, T., Bayliss, J.A., Lockie, S.H., Reichenbach, A., Lemus, M.B., Perello, M., Spencer, S.J., Kozicz, T., and Andrews, Z.B. (2016). Des-acyl ghrelin and ghrelin-o-acytransferase regulation hypothalamic-pituitary adrenal axis activation and anxiety in response to acute stress. Endocrinology, 157(10), 3946-3957.

Stevenson, J.R., Francomacaro, L.M., Bohidar, A.E., Young, K.A., Pesarchick, B.F., 
Buirkle, J.M., McMahon, E.K. and O'Bryan, C.M. (2016). Ghrelin receptor (GHS-R1A) antagonism alters preference for ethanol and sucrose in a concentration-dependent manner in prairie voles. Physiol Behav, 155, 231-236.

St-Onge, V. (2014). The role of ghrelin in reward based feeding. (Dissertation).

St-Onge V., Watts, A., and Abizaid, A. (2015). Ghrelin enhances cue-induced bar pressing for high fat food. Horm Behav, 78, 141-149.

Stricker, E.M. and Verbalis, J.G. (1990). Control of appetite and satiety: insights from biologic and behavioural studies. Nutr Rev, 48, 49-56.

Takaya, K., Ariyasu, H., Kanamoto, N., Iwakura, H., Yoshimoto, A., Harada, M. et al. (2000). Ghrelin strongly stimulates growth hormone release in humans. J Clin End Metab, 85, 4908-4911.

Tanaka, M., Nakahara, T., Kojima, S., Nakano, T., Muranaga, T., Nagai, N., Ueno, H., Nakazato, M., Nozoe, S. and Naruo, T. (2004). Effect of nutritional rehabilitation on circulating ghrelin and growth hormone levels in patients with anorexia nervosa. Regulatory Pept, 122(3), 163-168.

Tanaka, M., Naruo, T., Nagai, N., Kuroki, N., Shiiya, T., Nakazato, M., Matsukura, S. and Nozoe, S. (2003a). Habitual binge/purge behaviour influences circulating ghrelin levels in eating disorders. $J$ Psyhiatr Res, 37(1), 17-22.

Tanaka, M., Naruo, T., Yasuhara, D., Tatebe, Y., Nagai, N., Shiiya, T., Nakazato, M., Matsukura, S. and Nozoe, S. (2003b). Fasting plasma ghrelin levels in subtypes of anorexia nervosa. Psychoneuroendocrinology, 28(7), 829-835.

Terrill, S.J., Wall, K.D., Medina, N.D., Maske, C.B., and Williams, D.L. (2018). Lateral 
septum growth hormone secretagogue receptor affects food intake and motivation for sucrose reinforcement. Am J Physiol Regul Integr Comp Physiol, 315, R76R83.

Thomson, J.L. and Borgland, S.L. (2013). Presynaptic leptin action suppresses excitatory synaptic transmission onto ventral tegmental area dopamine neurons. Biol Psychiatry. 73(9), 860-868.

Troisi, A., Di Lorenzo, G., Lega, I., Tesauro, M., Bertoli, A., Leo, R., Iantorno, M., Pecchioli, C., Rizza, S., Turriziani, M., Lauro, R. and Siracusano, A. (2005). Plasma ghrelin in anorexia, bulimia, and binge-eating disorder: relations with eating patterns and circulating concentrations of cortisol and thyroid hormones. Neuroendocrinology, 81(4), 259-266.

Tschop, M., Smiley, D.L., and Heiman, M.L. (2000). Ghrelin induces adiposity in rodents. Nature, 407, 908-913.

Tuebner, B.J., Keen-Rhinehart, E. and Bartness, T.J. (2012). Third ventricular coinjection of subthreshold doses of NPY and AgRP stimulate food hoarding and intake and neural activation. Am J Physiol Regul Integr Comp Physiol., 302, R37-48.

Uchida, A., Zigman, J.M., and Perello, M. (2013). Ghrelin and eating behavior: evidence and insight from genetically-modified mouse models. Frontiers in Neuroscience, 7, 121, 1-10.

Ungerstedt, U. (1971). Adipsia and aphagia after 6-hydroxydopamine induce degeneration of the nigro-striatal dopamine system. Acta Physiol Scand Suppl., $367,95-122$.

Valdivia, S., Cornejo, M.P., Reynaldo, M., De Francesco, P.N. and Perello, M. (2015). 
Escalation of high fat intake in a binge eating model differentially engages dopamine neurons of the ventral tegmental area and requires ghrelin signaling. Psychoneuroendocrinology, 60, 206-216.

Valdivia, S., Patrone, A., Reynaldo, M. and Perello, M. (2014). Acute high fat diet consumption activates the mesolimbic circuit and requires orexin signaling in a mouse model. PLoS One, 9(1), e87478.

Valenstein, E.S., Cox, V.C., \& Kakolewski, J.W. (1968). Modification of motivated behavior elicited by electrical stimulation of the hypothalamus. Science. 159(819): 1119-1121.

van der Plasse, G., van Zessen, R., Luijendijk, M.C.M., Erkan, H., Studer, G.D., Ramakers G.M.J. and Adan, R.A.H. (2015). Modulation of cue-induced firing of ventral tegemental area dopamine neurons by leptin and ghrelin. International Journal of Obesity, 39, 1742-1749.

Vincent, M., Gao, G. and Jacobson, L. (2014). Comparison of the efficacy of five adenoassociated virus vectors for transducing dorsal raphe nucleus cells in the mouse. Journal of Neuroscience Methods, 235, 188-192.

Volkow, N.D., Wang, G.J., Fowler, J.S., and Telang, F. (2008). Overlapping neuronal circuits in addiction and obesity. Philos Tran R Soc Lond B Biiol Sci, 363, 31913200.

Volkow, N.D \& Wise, R.A. (2005). How can drug addiction help us understand obesity? Nature Neuroscience, 8(5), 555-560.

Walker, A.K., Ibia, I.E. and Zigman, J.M. (2012). Disruption of cue-potentiated feeding in mice with blocked ghrelin signaling. Physiol Behav, 108, 34-43. 
Wang, G.J., Volkow, N.D., Telang, F., Jayne, M., Ma, J., Rao, M., Zhu, W., Wong, C.T., Pappas, N.R. et al. (2004). Exposure to appetitive food stimuli markedly activates the human brain. Neuroimage, 21, 1790-1797.

Wayner, M.J. and Fraley, S. Enhancement of the consumption of acclimated sapid solutions following periodic and prolonged withdrawal (1972). Physiol Behav, 9(3), 463-474.

Wei, X.J., Sun, K., Chen, B., LV, X., Luo, X. and Yan, J.Q. (2015). Ghrelin signaling in the ventral tegmental area mediates both reward-based feeding and fastinginduced hyperphagia on high-fat diet. Neuroscience, 300, 53-62.

Wellman, M. and Abizaid, A. (2015). Knockdown of central ghrelin O-acyltransferase by vivo-morpholino reduced body mass of rats fed a high fat diet. Peptides, 70, 1722.

Wellman, P.J., Clifford, P.S., Rodriguex, J., Hughes, S., Eitan, S., Brunel, L., Fehrentz, J.A. and Martinez, J. (2011). Pharmacologic antagonism of ghrelin receptors attenuates the development of nicotine induced locomotor sensitization in rats. Regul Pept, 172(1-3), 77-80.

Wellman, P.J., Clifford, P.S., Rodriguez, J.A., Hughes, S., Di Francesco, C., Melotto, S., Tessari, M., Corsi, M., Bifone, A., and Gozzi, A. (2012). Brain reinforcement system function is ghrelin dependent studies I the rat using pharmacological fMRI and intracranial self-stimulation. Addict Biol, 17(5), 908-919.

Wise, R. (2006). Role of brain dopamine in food reward and reinforcement. Philos Trans R Soc Lond B Biol Sci, 361, 1149-1158.

Wise, R.A and Rompre. P.P. (1989). Brain dopamine and reward. Annu Rev Psychol, 40, 
191-225.

Wojnicki, F.H.E., Babbs, R.K., and Corwin R.L.W. (2013). Environments predicting intermittent shortening access reduce operant performance but not home cage binge size in rats. Physiology and Behavior, 116-117, 35-43.

Wojnicki, F.H.E., Babbs, R.K. and Corwin, R.L.W. (2010). Reinforcing efficacy of fat, as assessed by progressive ratio responding, depends upon availability not amount consumed. Physiology and Behavior, 100, 316-321.

Wolfer, D.P and Lipp, H. (2000). Dissecting the behaviour of transgenic mice: is it the mutation, the genetic background, or the environment. Experimental Physiology, $85.6,627-634$.

Wren, A.M., Small, C.J., Ward, H.L., Murphy, K.G., Dakin, C.L., Taheri, S., Kennedy, A.R., Roberts, G.H., Morgan, D.G., Ghatei, M.A., \& Bloom, S.R. (2000). The novel hypothalamic peptide ghrelin stimulates food intake and growth hormone secretion. Regulatory Peptides. 140: 148-152.

Wren, A.M., Small, C.J., Abbott, C.R., Dhillo, W.S., Seal, L.J., Cohen, M.A., Batterham, R.L., Taheri, S., Stanley, S.A., Ghatei, M.A., \& Bloom, S.R. (2001). Ghrelin causes hyperphagia and obesity in rats. Diabetes. 50: 2540-2547.

Wren, A.M., Seal, L.J., Cohen, M.A., Byrnes, A.E., Frost, G.S., Murphy, K.G., Dhillo, W.S., Ghatei, M.A., Bloom, S.R. (2001). Ghrelin enhances appetite and increases food intake in humans. J Clin Endo Metab, 86(12), 5992.

Yang, J., Brown, M.S., Liang, G., Grishin, N.V., Goldstein, J.L. (2008). Identification of the acyltransferase that octanoylates ghrelin, an appetite-stimulating peptide hormone. Cell, 132, 387-396. 
Zhang, Y., Proenca, R., Maffei, M., Barone, M., Leopold,L and Friedman, J.M. (1994). Positional cloning of the mouse obese gene and its human homologue. Nature, $372,425-432$.

Zhang, J.V., Ren, P.G., Avsian-Kretchmer, O., Luo, C.W., Rauch, R., Klein, C., et al. (2005). Obestatin, a peptide encoded by the ghrelin gene, opposes ghrelins effects on food intake. Science, 310, 996-999.

Zigman, J.M., Bouret, S.G., and Andrews, Z.B. (2016). Obesity impairs the action of the neuroendocrine ghrelin system. Trends Endocrinol Metab, 27(1),54-63.

Zigman, J.M., Jones, J.E., Lee, C.E., Saper, C.B., and Elmquist, J.K. (2006). Expression of ghrelin receptor mRNA in the rat and mouse brain. Journal of Comparative Neurology, 494, 528-548.

Zigmond, M.J. \& Stricker, E.M. (1972). Deficits in feeding behavior after intraventricular injections of 6-hydroxydopamine in rats. Science. 177(4055): 1211-1213.

Zizzari, P., Longchamps, R., Epelbaum, J. and Bluet-Pajot, M.T. (2007). Obestatin partially affects ghrelin stimulation of food intake and growth hormone secretion in rodents. Endocrinology, 148, 1648-1653. 